\title{
The origin of the escape of Lyman $\alpha$ and ionizing photons in Lyman Continuum Emitters
}

\author{
S. Gazagnes ${ }^{1,2}$, J. Chisholm ${ }^{3, \dagger}$, D. Schaerer ${ }^{2,4}$, A. Verhamme ${ }^{2}$, and Y. Izotov ${ }^{5}$ \\ 1 Kapteyn Astronomical Institute, University of Groningen, P.O Box 800, 9700 AV Groningen, The Netherlands \\ 2 Observatoire de Genève, Université de Genève, 51 Ch. des Maillettes, 1290 Versoix, Switzerland \\ 3 University of California-Santa Cruz, 1156 High Street, Santa Cruz, CA, 95064, USA \\ ${ }^{4}$ CNRS, IRAP, 14 Avenue E. Belin, 31400 Toulouse, France \\ 5 Bogolyubov Institute for Theoretical Physics, National Academy of Sciences of Ukraine, 14-b Metrolohichna str., Kyiv 03143, \\ Ukraine
}

Received $<$ date $>$ / Accepted $<$ date $>$

\begin{abstract}
Context. Identifying the physical mechanisms driving the escape of Lyman Continuum (LyC) photons is crucial to find Lyman Continuum Emitter (LCE) candidates.

Aims. To understand the physical properties involved in the leakage of $\mathrm{LyC}$ photons, we investigate the connection between the $\mathrm{H}_{\mathrm{I}}$ covering fraction, $\mathrm{H}_{\mathrm{I}}$ velocity width, the Lyman $\alpha(\operatorname{Ly} \alpha)$ properties and the escape of LyC photons in a sample of 22 star-forming galaxies including 13 confirmed LCEs.

Methods. We fit the stellar continuum, dust attenuation, and absorption lines between $920 \AA$ and $1300 \AA$ to extract the H $\mathrm{I}$ covering

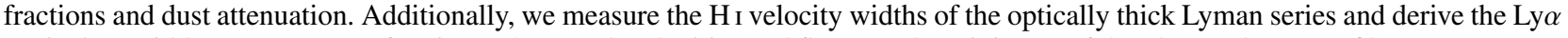
equivalent widths $(\mathrm{EW})$, escape fractions $\left(f_{\text {esc }}\right)$, peak velocities and fluxes at the minimum of the observed Ly $\alpha$ profiles.

Results. Overall, we highlight strong observational correlations between the presence of low $\mathrm{H}$ i covering fractions and the observation of (1) low Ly $\alpha$ peak velocities; (2) more flux at the profile minimum; and (3) larger EW(Ly $\alpha), f_{\text {esc }}(\operatorname{Ly} \alpha)$, and $f_{\text {esc }}^{\text {obs }}($ LyC). Hence, low column density channels are crucial ISM ingredients for the leakage of Ly $\alpha$ and LyC photons. Additionally, galaxies with narrower $\mathrm{H}_{\mathrm{I}}$ absorption velocity widths have higher $\operatorname{Ly} \alpha$ equivalent widths, larger Ly $\alpha$ escape fractions, and lower Ly $\alpha$ peak velocity separations. This may suggest that these galaxies have lower H I column densities. Finally, we find that dust also regulates the amount of Ly $\alpha$ and LyC radiation that actually escapes the ISM.

Conclusions. The ISM porosity is one origin of strong $\mathrm{Ly} \alpha$ emission and enables the escape of ionizing photons in low-z leakers. However, this is not enough to explain the largest $f_{\mathrm{esc}}^{\mathrm{obs}}(\mathrm{LyC})$, which indicates that the most extreme LCEs are likely density-bounded along all lines of sight to the observer. Overall, the neutral gas porosity constrains a lower limit to the escape fraction of $\mathrm{LyC}$ and $\mathrm{Ly} \alpha$ photons, providing a key estimator of the leakage of ionizing photons.
\end{abstract}

Key words. galaxies: ISM - ISM: abundances - ISM: lines and bands - Ultraviolet: ISM - dust, extinction - dark ages, reionization, first stars

\section{Introduction}

The Epoch of Reionization (EoR) is a key transition phase in the history of the Universe, which is still largely unconstrained from observations. In the upcoming era of large telescopes, numerous galaxies within the EoR will be observed, but determining the mechanisms responsible for the propagation of the ionizing radiation from the interstellar medium (ISM) to the intergalactic medium (IGM) is crucial. Although the ionizing contribution of AGN during reionization is actively discussed (Fontanot et al. 2012, 2014; Robertson et al.|2015; Madau \& Haardt|2015; Mitra et al.2018), many studies suggest that a population of low-mass star-forming galaxies with an average escape fraction of Lyman Continuum (LyC) photons of 10-20\% probably dominates the contribution to the ionizing budget of the EoR (Ouchi et al. 2009 Robertson et al. 2013, Dressler et al. 2015, Finkelstein et al. 2019). However, the quest to find LyC leaking galaxies at high redshift $(z>6)$ is very challenging, and direct observations of

\footnotetext{
Send offprint requests to: s.r.n.gazagnes@ rug.nl

$\dagger$ Hubble Fellow
}

the ionizing flux at $\lambda<912 \AA$ are complicated (or statistically unfeasible) due the attenuation of the IGM and the presence of interlopers along the line of sight.

These caveats have been overcome by searches for compact, low-mass star-forming galaxies in the local universe, which can serve as analogs, and significant progress has been made over the past few years, with successful detections of LyC leakage at $\mathrm{z}<0.5$ (15 individual detections, Bergvall et al. 2006; Leitet et al. 2013, Borthakur et al. 2014, Leitherer et al. 2016; Izotov et al. 2016a b, 2018a b), and at z 2-3 (Vanzella et al. 2015, de Barros et al.|2016; Shapley et al.|2016; Bian et al.| 2017; Steidel et al.2018; Fletcher et al.|2019, Rivera-Thorsen et al.|2019). This recent breakthrough has provided an ideal laboratory to explore the physical properties that favor the leakage of ionizing radiation in the LyC leakers.

Studies commonly refer to two major physical models to understand how LyC photons escape galaxies (see e.g. Zackrisson et al. 2013). In a density-bounded ISM, the H I column density $\left(N_{\mathrm{H}_{\mathrm{I}}}\right)$ surrounding the stellar populations is too low $(<$ $10^{17.9} \mathrm{~cm}^{-2}$ ) to efficiently absorb all the ionizing photons passing through the neutral clouds, and the fraction of LyC radiation 
that escapes the ISM is proportional to the residual $N_{\mathrm{H} \text { I }}$ in the galaxy. Conversely, in a picket-fence or ionization bounded system, the bulk of the stars is surrounded by an optically thick ISM, where the average $N_{\mathrm{H}_{\mathrm{I}}}$ is high enough to efficiently absorb the LyC photons. In this model, ionizing photons escape through privileged paths through the ISM, referred to as holes or channels, which have no or little H I column densities. This scenario is mainly characterized by the porosity of the neutral gas, which is defined as the fraction of all sightlines to all of the ensemble of far-UV continua sources that are "covered" by Hi gas with column densities above a certain value. One way to define this $\mathrm{H}_{\mathrm{I}}$ "covering fraction" $\left(C_{f}\left(\mathrm{H}_{\mathrm{I}}\right)\right)$ is by using a set of $\mathrm{H}_{\mathrm{I}}$ absorption lines in the far-UV that have a range of oscillator strengths and saturate above a certain column density (between $\sim 10^{15}$ and $10^{16} \mathrm{~cm}^{-2}$ for the range from $\operatorname{Ly} \beta$ to Ly7). The range of oscillator strengths allows for simultaneous optical depth and covering fraction determination of the Lyman series lines. The H I covering fraction measured using this approach is always a lower limit to the true geometric covering fraction of the neutral gas, because of potential kinematic effects (Jones et al. 2013; RiveraThorsen et al. 2015, Vasei et al. 2016), or the presence of $\mathrm{H}_{\mathrm{I}}$ residuals (Kakiichi \& Gronke 2019). Nevertheless, in this paper, we use this measure of $C_{f}(\mathrm{HI})$ as a proxy of the overall neutral gas porosity and study how that porosity relates to the escape of Ly $\alpha$ and LyC photons.

Investigating which model represents the ISM of LCEs is also crucial to find indirect tracers of the leakage of ionizing photons. To do this, low-redshift observations remain the ideal approach. Indeed, far-UV spectroscopic observations are less likely to be contaminated by line-of-sight absorbers in the IGM, thus their LyC escape fraction can be measured directly from the flux at $\lambda<912 \AA$. Additionally, the study of their UV H I and metal lines constrain their neutral gas properties and identify the processes driving the LyC leakage. Reliable LyC probes have already been identified by studying the Ly $\alpha$ properties (see e.g. Verhamme et al. 2017; Izotov et al. 2018b, 2020).

Ly $\alpha$ also provides powerful insights on the distribution and kinematics of the neutral gas, and is closely related to the LyC properties. Indeed, the ionizing radiation arising from young massive stars creates $\mathrm{H}$ II regions surrounding the star-forming clusters, which produces Ly $\alpha$ radiation due to the recombination of hydrogen atoms. Ly $\alpha$ photons are resonantly scattered as they travel through the neutral gas, and the amount of scattering events, which is determined by the $\mathrm{H}_{\mathrm{I}}$ gas distribution and column density, strongly impacts the shape of the Ly $\alpha$ profile. Since Ly $\alpha$ and LyC photons interact with the same neutral gas, the underlying physical mechanisms driving their leakage should be closely connected.

Nevertheless, this is not trivial because the Ly $\alpha$ transition is resonant. While LyC photons cannot escape from optically thick ISM, Ly $\alpha$ photons pass through dense neutral clouds by being scattered out of the velocity range covered by the neutral gas. The theoretical connection between Ly $\alpha$ and LyC has first been investigated using radiative transfer models (Verhamme et al. 2015; Dijkstra et al. 2016). These studies highlighted that the Ly $\alpha$ spectral shape provides insights on the H I column density and/or the existence of holes in the neutral gas spatial distribution. The presence of paths entirely cleared of H I gas in the ISM should imprint a single Ly $\alpha$ peak emission at the systemic, nevertheless, the presence of a double-peaked Ly $\alpha$ profile with a narrow peak separation in all the confirmed LCEs suggested that they have density-bounded ISMs (Verhamme et al. 2017; Izotov et al. 2018b). However, a follow-up study revealed the presence of saturated Lyman series with non-unity covering fraction (Gazagnes et al. 2018). This outcome favors an ionization bounded model with holes in an optically thick interstellar medium, hence at first sight incompatible with their doublepeaked Ly $\alpha$ profile. Additionally, Steidel et al. (2018) reported a correlation between the $\mathrm{EW}(\mathrm{Ly} \alpha)$ and the Hi covering fraction in stacks of $\mathrm{z} \approx 3$ galaxies, while McKinney et al. (2019) found a significant trend connecting the escape fraction of Ly $\alpha$ photons and the $\mathrm{Si}$ il covering fraction in a sample of extreme Green Peas (GPs). Hence, the latest studies suggest that the ISM is likely a very complex environment, and improved Ly $\alpha$ radiative transfer models are needed to constrain the origin of the connection between the Ly $\alpha$ spectral shape and the escape of LyC photons.

Promising insights were recently found by Ly $\alpha$ simulation studies showing that a very clumpy distribution of neutral gas, or low-density channels produced by turbulence could favor the leakage of LyC photons and create a double peak Ly $\alpha$ profile with low $v_{\text {Ly } \alpha}^{\text {sep }}$ (Gronke et al. 2016, 2017, Kimm et al. 2019; Kakiichi \& Gronke 2019). On the other hand, Jaskot et al. (2019) recently proposed that different Ly $\alpha$ markers probe different ISM properties; the peak separation could trace the presence of lowdensity gas, while the covering fraction relates to the gas porosity. Hence, further clarifications of the physical properties of the neutral gas of known LCEs are crucial to test the reliability of the $\mathrm{Ly} \alpha$-LyC correlations, and to investigate the accuracy of indirect $f_{\text {esc }}(\mathrm{LyC})^{1}$ predictions that use the $\mathrm{H}_{\mathrm{I}}$ covering fraction (Chisholm et al. 2018).

In this work, we investigate the connection between the ISM porosity (characterised by the $\mathrm{H}_{\mathrm{I}}$ covering fraction), the Ly $\alpha$ properties, and the LyC escape fraction in an unique sample of 22 star-forming galaxies, including 13 confirmed LCEs, which have neutral hydrogen Lyman series and Ly $\alpha$ observations. While we focus primarily on the presence of (dusty) lowdensity channels to explain the escape of LyC and Ly $\alpha$ photons, we also explore the impact of the width of saturated H I absorption lines on the spectral shape of the Ly $\alpha$ profile.

This paper is organized as follows: Sect.2 describes the observational data. Section 3 defines the methods used to measure the neutral gas and Ly $\alpha$ properties of the galaxies in our sample. In Sect. 4, we compare and discuss the connection between the spatial distribution and kinematics of neutral gas on both the Ly $\alpha$ properties and the escape of LyC photons. Section 5 discusses how the porosity of the neutral gas triggers the LyC leakage, and how it can be used to provide a lower limit to the total escape fraction of LyC photons in high-z galaxies. Finally, we summarize the main conclusions from this work in Sect. 6 .

\section{Data}

In this work, we investigate the relation between the neutral gas covering fraction, Ly $\alpha$ properties, and the escape of LyC photons in the sample of 22 star-forming galaxies listed in Table 1. We selected these galaxies because they have publicly available rest-frame UV spectroscopy both for Ly $\alpha$ and for the rest of the Lyman series (i.e between Lyman- $\beta$ at $1025 \AA$ and the Lyman limit at $912 \AA$ ). The latter can be observed with a spectral resolution $\mathrm{R}>1500$ for galaxies at $\mathrm{z}>0.18$ with the Cosmic Origins Spectrograph (COS) onboard the Hubble Space Telescope (HST) (Green et al. 2012). The relation between the neutral gas properties and the escape of ionizing photons was already explored for 16 of the 22 galaxies (Gazagnes et al. 2018, Chisholm et al. 2018). This includes 13 low redshift galaxies

\footnotetext{
1 In this paper, unless stated otherwise, $f_{\mathrm{esc}}(\mathrm{LyC})$ refers to the escape of ionizing photons along the line of sight.
} 
Table 1. Properties of galaxies with Lyman series observations.

\begin{tabular}{llll}
\hline \hline Galaxy name & $z$ & $12+\log (\mathrm{O} / \mathrm{H})$ & $f_{\mathrm{esc}}^{\mathrm{obs}}(\mathrm{LyC})$ \\
& & & \\
$(1)$ & $(2)$ & $(3)$ & $(4)$ \\
\hline $\mathrm{J} 1243+4646$ & 0.4317 & $7.89^{a}$ & $0.726^{a}$ \\
$\mathrm{~J} 1154+2443$ & 0.3690 & $7.65^{b}$ & $0.460^{b}$ \\
$\mathrm{~J} 1256+4509$ & 0.3530 & $7.87^{a}$ & $0.380^{a}$ \\
$\mathrm{~J} 1152+3400$ & 0.3419 & $8.00^{c}$ & $0.132^{c}$ \\
$\mathrm{~J} 1011+1947$ & 0.3322 & $7.99^{a}$ & $0.114^{a}$ \\
$\mathrm{~J} 1442-0209$ & 0.2937 & $7.93^{c}$ & $0.074^{c}$ \\
$\mathrm{~J} 0925+1409$ & 0.3013 & $7.91^{d}$ & $0.072^{d}$ \\
$\mathrm{~J} 1503+3644$ & 0.3537 & $7.95^{c}$ & $0.058^{c}$ \\
$\mathrm{~J} 1333+6246$ & 0.3181 & $7.76^{c}$ & $0.056^{c}$ \\
$\mathrm{~J} 0901+2119$ & 0.2993 & $8.16^{a}$ & $0.027^{a}$ \\
$\mathrm{~J} 1248+4259$ & 0.3629 & $7.64^{a}$ & $0.022^{a}$ \\
$\mathrm{~J} 0921+4509$ & 0.23499 & $8.67^{e}$ & $0.010^{f}$ \\
Tol1247-232 & 0.0488 & $8.10^{g}$ & $<0.004^{h}$ \\
$\mathrm{~J} 0926+4427$ & 0.18069 & $8.01^{i}$ & - \\
$\mathrm{J} 1429+0643$ & 0.1736 & $8.20^{i}$ & - \\
GP0303-0759 & 0.16488 & $7.86^{i}$ & - \\
GP1244+0216 & 0.23942 & $8.17^{i}$ & - \\
GP1054+5238 & 0.25264 & $8.10^{i}$ & - \\
GP0911+1831 & 0.26223 & $8.00^{i}$ & - \\
SGAS J1226 & 2.92525 & - & - \\
SGAS J1527 & 2.76228 & $<8.5^{j}$ & - \\
Cosmic Eye & 3.07483 & $8.60^{k}$ & \\
\hline \hline & & & - \\
\hline
\end{tabular}

Notes. (1) Galaxy name; (2) redshift; (3) metallicities derived from oxygen optical emission lines; (4) observed Lyman continuum escape fraction derived from SED fitting. Dashes indicate that the quantities have not been measured. The reference studies for the metallicities and LyC escape fractions are listed below.

References. (a) Izotov et al. (2018b) (b) Izotov et al. (2018a); (c) Izotov et al. (2016b); (d) Izotov et al. (2016a); (e) Pettini \& Pagel (2004); (f) Borthakur et al. (2014); (g) Leitherer et al. (2016); (h) Chisholm et al. (2017a); (i) Izotov et al. (2011); (j) Stark et al. (2008); (k) Wuyts et al. (2012).

$(\mathrm{z}<0.4), 7$ of which are confirmed LyC emitting galaxies; $\mathrm{J} 0925+1409$, J1503+3644, J1152+3400, J1333+6246, J14420209 from Izotov et al. (2016a b), J0921+4509 from Borthakur et al. (2014) and Tol1247-232 from Leitherer et al. (2016). Four of them are Green Peas (GP) (Henry et al. 2015), 2 are Lyman break analogs (LBA) (Heckman et al. 2011, 2015) and the 3 remaining galaxies are gravitationnally lensed galaxies at $\mathrm{z} \approx 3$ (SGAS J122651.3+215220, SGAS J152745.1+065219, and the Cosmic Eye, Stark et al. 2008; Koester et al. 2010) from the Magellan Evolution of Galaxies Spectroscopic and Ultraviolet Reference Atlas (MegaSaura; Rigby et al. 2018). Additionally, our sample includes 6 new galaxies that were not included in Gazagnes et al. (2018) and are the recently discovered low redshift $(0.2<\mathrm{z}<0.5)$ LyC emitting galaxies J1154+2443, $\mathrm{J} 1243+4646$, J1256+4509, J1011+1947，J0901+2119， and J1248+4249 from Izotov et al. (2018a b); three of which have extreme LyC escape fractions of 38, 46 and $72.6 \%$.

Nineteen of the galaxies observed with HST/COS are at such redshift that at least the $\operatorname{Ly} \beta$ line is observable with the COS
G140L or G130M gratings. The resolving power (R) of the restframe UV spectra around the Lyman series is $\approx 1500$ for all the confirmed leakers except that J0921+4509 which has a R $\approx 15000 \mathrm{G} 130 \mathrm{M}$ spectra. The $4 \mathrm{GPs}$ and the 2 LBAs have observations of one or several $\mathrm{H}_{\mathrm{I}}$ absorption lines with a spectral resolution of $\approx 15000$. Additionally, all the Ly $\alpha$ profiles were observed with the medium resolution grating G160M $(R \approx 16000$ at $1600 \AA$ ). The data for the 13 leakers were reduced using CALCOSv2.21 and a custom method for faint COS spectra (Worseck et al. 2016). The other COS/HST data were reduced with CALCOSv2.20.1 and the methods from Wakker et al. (2015). The three MegaSaura galaxies have been observed with the MagE spectrograph on the Magellan Telescopes (Marshall et al. 2008), and have moderate resolution spectroscopy $(R \sim 3000)$ both for their Lyman series and $\operatorname{Ly} \alpha$. They are the only galaxies in the MegaSaura sample with a signal-to-noise ratio $(\mathrm{S} / \mathrm{N})$ sufficient $(>2)$ to constrain their neutral gas properties with the Lyman series. In this paper, we used the following short names for the two sources: SGAS J122651.3+215220 = SGAS J1226 and SGAS $\mathrm{J} 152745.1+065219=$ SGAS J1527.

Table 1] summarizes the galaxy properties of the sample. The metallicities have been derived from the optical [O III] $4366 \AA$ oxygen emission lines, using the direct $T_{e}$ method, in all the low redshift galaxies. The metallicity of the Cosmic Eye has been measured by Stark et al. (2008) using the $\mathrm{R}_{23}$ index, and an upper limit has been derived from the $[\mathrm{N}$ II $] / \mathrm{H} \alpha$ ratio for SGAS $\mathrm{J} 1527(12+\log (\mathrm{O} / \mathrm{H})<8.5$; Wuyts et al. 2012). The metallicity of SGAS J1226 has not been measured because these lines are not accessible from the ground. Several different measurements of the escape fraction of ionizing photons from Tol1247232 are reported in the literature: $4.2 \pm 1.2 \%$ in Leitherer et al. (2016), $<0.4 \%$ in Chisholm et al. (2017a) and $1.5 \pm 0.5 \%$ in Puschnig et al. (2017). We used the value derived in Chisholm et al. (2017a) since the measurement method is consistent with the one used for the other leakers.

\section{Method}

We now describe the methods used to study the ISM and Ly $\alpha$ properties of the 22 galaxies in our sample.

\subsection{Neutral gas properties}

To measure the $\mathrm{H}_{\mathrm{I}}$ velocity shift and covering fraction of the galaxies, we used the approach detailed in Gazagnes et al. (2018), and recall here the main steps. We first correct the galaxy spectra for Milky Way extinction using the Cardelli et al. (1989) extinction law, $\mathrm{R}(\mathrm{V})=3.1$, and the galactic $E_{\mathrm{B}-\mathrm{V}}$ reported in the NASA Extragalactic Database $\left(\mathrm{NED}^{2}\right)$. We then fit the stellar continuum as in Chisholm et al. (2019), including dust extinction, metal and $\mathrm{H}$ I absorption lines to consistently determine the UV attenuation in the galaxy, as well as the column densities and covering fractions of the individual ions. The stellar continuum model, $F^{\star}$, is a linear combination of 50 single-age fully theoretical stellar continuum models with 5 metallicities, 0.05, 0.2, $0.4,1$, and $2 Z_{\odot}$, with ages of $1,2,3,4,5,8,10,15,20$, and $40 \mathrm{Myr}$, drawn from the STARBURST99 library (S99; Leitherer et al. 1999). This is numerically given as:

$F^{\star}=\Sigma_{i=1}^{50} X_{i} F_{i}^{S 99, Z_{\mathrm{i}}}$

${ }^{2}$ https://ned.ipac.caltech.edu 
Table 2. Dust extinction and $\mathrm{H}_{\mathrm{I}}$ properties derived from the Lyman series.

\begin{tabular}{|c|c|c|c|c|}
\hline Galaxy name & $\begin{array}{l}E_{\mathrm{B}-\mathrm{V}} \\
{[\mathrm{mag}]} \\
(2)\end{array}$ & $C_{f}\left(\mathrm{H}_{\mathrm{I}}\right)$ & $\begin{array}{l}v_{\mathrm{H}_{\mathrm{I}}}^{\text {shift }} \\
{\left[\mathrm{km} \mathrm{s}^{-1}\right]} \\
(4)\end{array}$ & $\begin{array}{l}v_{\mathrm{H} \text { I }}^{\text {width }} \\
{\left[\mathrm{km} \mathrm{s}^{-1}\right]} \\
(5)\end{array}$ \\
\hline $\mathrm{J} 1243+4646$ & $0.100 \pm 0.021$ & $<0.189$ & - & - \\
\hline $\mathrm{J} 1154+2443$ & $0.118 \pm 0.031$ & $0.450 \pm 0.086$ & $-289 \pm 92$ & $170 \pm 86$ \\
\hline $\mathrm{J} 1256+4509$ & $0.076 \pm 0.029$ & $0.409 \pm 0.079$ & $-48 \pm 44$ & $250 \pm 50$ \\
\hline $\mathrm{J} 1152+3400 *$ & $0.144 \pm 0.021$ & $0.625 \pm 0.054$ & $-346 \pm 28$ & $419 \pm 60$ \\
\hline J1442-0209 & $0.140 \pm 0.015$ & $0.556 \pm 0.038$ & $-261 \pm 34$ & $371 \pm 53$ \\
\hline J0925+1409 & $0.164 \pm 0.015$ & $0.638 \pm 0.086$ & $-214 \pm 151$ & $320 \pm 60$ \\
\hline $\mathrm{J} 1011+1947$ & $0.230 \pm 0.084$ & $0.708 \pm 0.113$ & $-69 \pm 32$ & $285 \pm 54$ \\
\hline $\mathrm{J} 1503+3644 *$ & $0.217 \pm 0.014$ & $0.721 \pm 0.055$ & $-79 \pm 24$ & $356 \pm 49$ \\
\hline $\mathrm{J} 1333+6246$ & $0.151 \pm 0.043$ & $0.804 \pm 0.058$ & $-126 \pm 48$ & $280 \pm 51$ \\
\hline J0901+2119 & $0.220 \pm 0.057$ & $0.637 \pm 0.166$ & $-121 \pm 84$ & $300 \pm 78$ \\
\hline $\mathrm{J} 1248+4259$ & $0.253 \pm 0.073$ & $0.954 \pm 0.104$ & $56 \pm 49$ & $232 \pm 50$ \\
\hline J0921+4509 & $0.222 \pm 0.015$ & $0.761 \pm 0.080$ & $-56 \pm 13$ & $440 \pm 20$ \\
\hline Tol1247-232* & $0.195 \pm 0.028$ & $0.518 \pm 0.046$ & $194 \pm 41$ & $453 \pm 89$ \\
\hline J0926+4427* & $0.175 \pm 0.010$ & $0.768 \pm 0.034$ & $-199 \pm 12$ & $383 \pm 46$ \\
\hline J1429+0643* & $0.165 \pm 0.020$ & $0.931 \pm 0.046$ & $-220 \pm 36$ & $420 \pm 50$ \\
\hline GP0303-0759 & $0.121 \pm 0.045$ & $0.908 \pm 0.207$ & $-266 \pm 92$ & $380 \pm 50$ \\
\hline GP1244+0216 & $0.290 \pm 0.043$ & $0.946 \pm 0.123$ & $-78 \pm 48$ & $379 \pm 49$ \\
\hline GP1054+5238* & $0.253 \pm 0.054$ & $0.823 \pm 0.101$ & $-161 \pm 29$ & $480 \pm 29$ \\
\hline GP0911+1831 & $0.352 \pm 0.038$ & $0.752 \pm 0.092$ & $-273 \pm 40$ & $374 \pm 16$ \\
\hline SGAS J1226 & $0.201 \pm 0.001$ & $0.994 \pm 0.009$ & $-264 \pm 21$ & $548 \pm 29$ \\
\hline SGAS J1527* & $0.314 \pm 0.002$ & $0.990 \pm 0.034$ & $-247 \pm 25$ & $480 \pm 30$ \\
\hline Cosmic Eye* & $0.371 \pm 0.006$ & $0.990 \pm 0.023$ & $311 \pm 16$ & $467 \pm 99$ \\
\hline
\end{tabular}

Notes. (1) Galaxy name; (2) dust attenuation parameter ; (3) H I covering fraction; (4) H I velocity shift; (5) H I velocity width of maximal absorption. * changes with respect to Gazagnes et al. (2018), see Sect. 3 for details.

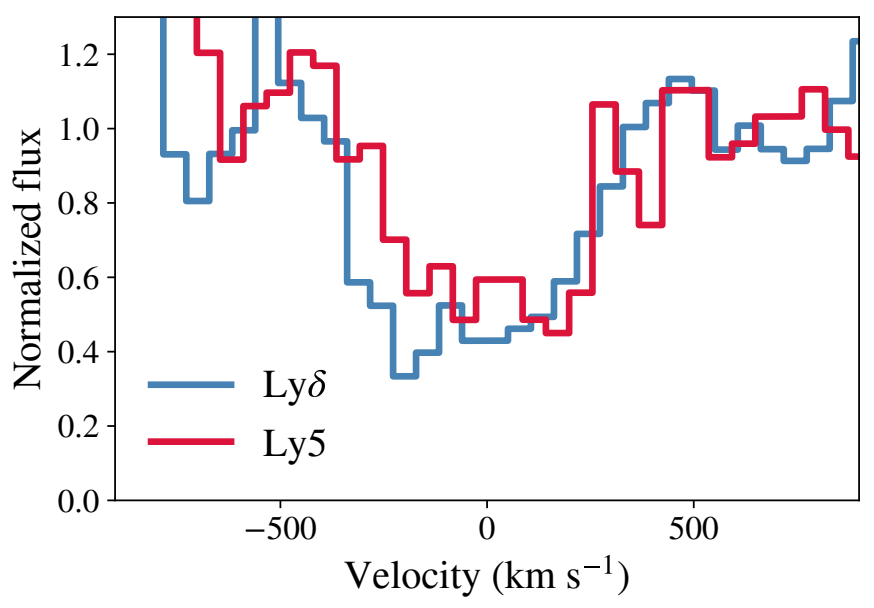

Fig. 1. Plot of the Ly $\delta$ (blue) and Ly5 (red) absorption lines in the galaxy $\mathrm{J} 1256+4509$. The flux has been normalized using the median of the observed spectra taken between 500 and $1000 \mathrm{~km} \mathrm{~s}^{-1}$ from the absorption lines. The two $\mathrm{H}_{\mathrm{r}}$ absorption lines have similar depth and width, which indicates that they are saturated.

where $X_{i}$ and $F_{i}^{99, Z_{\mathrm{i}}}$ are respectively the linear coefficients and the STARBURST99 theoretical stellar continuum models for a given age and metallicity. The S99 spectra were computed with the WM-Basic spectral library (Leitherer et al. 2010), using a
Kroupa initial mass function with a high and low mass exponent of 2.3 and 1.3 respectively, a high-mass cutoff of $100 \mathrm{M}_{\odot}$, and the stellar evolution tracks with high mass loss from Meynet et al. (1994). Additionally, the large amount of ionizing photons produced by young massive stars produces free-free, free-bound, and two-photon nebular continuum emission, can have a significant impact on the total continuum flux in young, low metallicity stellar populations (Steidel et al. 2016; Byler et al. 2018). Following the procedure detailed in Chisholm et al. (2019), we created a nebular continuum for each single-age and metallicity stellar model using Cloudy v17.0 (Ferland et al. 2013, 2017), assuming similar gas-phase metallicity and stellar metallicity, a volume hydrogen density of $100 \mathrm{~cm}^{-3}$ and an ionization parameter $\log (\mathrm{U})=-2.5$. The final output nebular continua were added to the stellar models and the final synthetic spectra have a spectral resolution $\mathrm{R} \approx 2500$, which is convolved to the resolution of the data. We used the far-UV dust attenuation curve from Reddy et al. (2016a) and an uniform dust screen model to account for the dust attenuation.

Absorption lines from different ions were included using Voigt profiles defined by 4 free parameters: the velocity shift $\left(v^{\text {shift }}\right)$, the Doppler parameter $(b)$, the column density $(N)$, and the covering fraction $\left(C_{f}\right)$. The linear combination of stellar continuum models, interstellar absorption lines, and dust attenuation produces the final fitted spectrum.

As we are interested in the Lyman series, the spectral region that we fit is taken from 912 to $1050 \AA$. We include redder 
portions of the spectrum, up to $1300 \AA$, to further constrain the stellar model and dust attenuation. We aimed at including all the $\mathrm{H}_{\mathrm{I}}$ absorption lines that are observed to improve the constraints on the Hi parameters. Nevertheless, in practice, the wavelength regime from 912 to $930 \AA$ i were excluded due to low $\mathrm{S} / \mathrm{N}$, and/or geocoronal emission. Additionally, $\operatorname{Ly} \beta$ is not systematically fit since it is located close to a strong O vi P-Cygni profile, which synthetic stellar models sometimes fail to reproduce (see the fits in Appendix A, and often decreases the fit quality when combined with bluer $\mathrm{H}_{\mathrm{I}}$ absorption lines. The $\mathrm{O}_{\mathrm{I}}$ absorption lines that directly blend with the Lyman series are always included, and their parameters are mostly constrained by the O I 989 and $1039 \AA$ lines. However, because of low $\mathrm{S} / \mathrm{N}$ and/or low $N_{\text {OI }}$, the latter are not always resolved, such that we cannot accurately recover the $\mathrm{O}_{\mathrm{I}}$ contribution in these galaxies. Finally, absorption lines from $\mathrm{O}_{\text {VI, }} \mathrm{Si}$ II, C II, C III or from the Milky Way are sometimes added, provided that they improve the fit around the Lyman series. For more details see Gazagnes et al. (2018).

Assuming a foreground dust attenuation, the final fitted model, $F_{\text {mod }}(\lambda)$, can be expressed as

$F_{\text {mod }}(\lambda)=F^{\star}(\lambda) \times 10^{-0.4 E_{\mathrm{B}-\mathrm{v}} k_{\text {Reddy } 16}(\lambda)} \times \mu_{\text {ion }}(\lambda)$

where $\mu_{\text {ion }}(\lambda)$ represents the fitted profiles of absorption lines given by:

$\mu_{\text {ion }}(\lambda)= \begin{cases}1-C_{f}(\text { ion })+C_{f}(\text { ion }) \times \exp ^{-\tau_{\text {ion }}(\lambda)} & \text { if included } \\ 1 & \text { otherwise }\end{cases}$

Equation (2) assumes that all the photons escaping the ISM are affected by the same dust extinction. In practice, galaxies include several star-forming clumps, which might not be affected by the same dust attenuation. Thus, the recovered $E_{\mathrm{B}-\mathrm{V}}$ should be interpreted as the mean extinction in the galaxy.

The fitting method is based on an IDL routine that uses nonlinear least squares fitting, MPFIT (Markwardt 2009), and returns the best fit parameters and their statistical errors for each free parameter fitted. In this work, we focus mainly on the fitted $\mathrm{H}_{\mathrm{I}}$ parameters: $v_{\mathrm{H}_{\mathrm{I}}}^{\text {shift }}, N_{\mathrm{H}_{\mathrm{I}}}$ and $C_{f}(\mathrm{HI})$. As discussed in Jones et al. (2013), Rivera-Thorsen et al. (2015) and Vasei et al. (2016), the interpretation of the measured $C_{f}$ must be taken with caution. This is because the kinematics of the absorbing gas impacts the observed depth of the absorption lines. Indeed, two dense $\mathrm{H}_{\mathrm{I}}$ clouds with non-overlapping velocity distributions and each covering half of the galaxy will imprint $\mathrm{H}_{\mathrm{I}}$ absorption lines with $C_{f}(\mathrm{HI})=0.5$. However, in this case, the total geometrical covering fraction is 1 because $\mathrm{LyC}$ photons are insensitive to kinematic effects (the $\mathrm{H}_{\mathrm{I}}$ absorbs ionizing photons at all wavelengths below the Lyman limit). Hence, $C_{f}\left(\mathrm{H}_{\mathrm{I}}\right)$ is always a lower limit to the covering fraction seen by the ionizing photons. Nevertheless, we showed in Gazagnes et al. (2018) that it seems to be a good proxy to the true geometric covering fraction of the optically thick H I clouds in the current sample.

In Gazagnes et al. (2018), we used simulations to show that the $N_{\mathrm{H}_{\mathrm{I}}}$ derived from the fits suffers from large uncertainties due to the degeneracy between the Doppler parameter $b$ and the column density when the absorption lines are saturated. The typical resolution and $\mathrm{S} / \mathrm{N}$ of the observations is too low to constrain $N_{\mathrm{H}_{\mathrm{I}}}$ directly from the Lyman series. Consequently, we neither report nor use the $N_{\mathrm{H}_{\mathrm{I}}}$ and $b$ values derived using our fitting procedure. Alternatively, one can indirectly estimate $N_{\mathrm{H}_{\mathrm{I}}}$ using an approach based on $N_{\mathrm{O}_{\text {I }}}$ and the metallicity, $12+\log (\mathrm{O} / \mathrm{H})$. Nevertheless, $\mathrm{O}_{\mathrm{I}}$ is not detected in 6 galaxies, which might be due to low $\mathrm{S} / \mathrm{N}$, small $N_{\mathrm{O}_{\text {I }}}$ or low covering fraction. Conversely, we established in Gazagnes et al. (2018) that the H i covering fraction can be inferred with a reasonable accuracy from Voigt fitting methods given the spectral resolution and $\mathrm{S} / \mathrm{N}$ of the galaxies observed in our sample. A systematic error, relative to the resolution and $\mathrm{S} / \mathrm{N}$ of a given spectrum, needs to be included in the final error term because the statistical error returned by MPFIT does not account for it (see Sect 2 and Table 3 in Gazagnes et al. 2018). The typical systematic error of the covering fraction derived from spectra with $\mathrm{R}=1500$ (typical resolution of the GL140 grating) and S/N $\approx 2$ is 0.10 . Note that this typical systematic error is only valid if the Lyman series lines are saturated. When the $\mathrm{H}$ I absorption lines are not saturated, the residual flux at the bottom of an absorption line is similarly impacted by the presence of low $N_{\mathrm{H} \text { I }}$ and/or low $C_{f}(\mathrm{HI})$, such that an accurate constraint on these parameters would require higher $\mathrm{S} / \mathrm{N}$ and resolution.

Fig. 2 shows the observed flux, the error on the flux, and the best fit obtained for the galaxy J1256+4509 using our fitting approach. It highlights the main regions masked during the fits, either due to geo-coronal emission, low $\mathrm{S} / \mathrm{N}$, Ly $\alpha$ emission, or ISM or Milky Way absorption lines that are not fit. We estimate an uncertainty on the fit using a Monte-Carlo approach where the observed flux is modified by a Gaussian kernel centered on zero with standard deviation corresponding to the error on the flux. We performed 100 fit realizations and took the standard deviation as the error of the fit (represented by a blue shaded area in the figure). Figure 2 shows that this uncertainty is roughly on the order of the error of the observed flux in the highest $\mathrm{S} / \mathrm{N}$ regions. Interestingly, the error is low around the Lyman series lines, suggesting that the latter are robustly constrained and little affected by fluctuations in the fitted stellar continuum. This point is further discussed in Sect. 3.2. where we emphasize the complexity of constraining the stellar continuum and dust extinction in galaxy spectra with low $\mathrm{S} / \mathrm{N}$. Section $\mathrm{A}$ presents the fits obtained for the 5 other leakers from Izotov et al. (2018a b), the 16 remaining fits can be found in Gazagnes et al. (2018).

Fitting the Lyman series using a Voigt profile assumes that the lines follow a single Gaussian velocity distribution, and this assumption might not be valid for absorption profiles arising from galactic outflows (Heckman et al. 2000; Pettini et al. 2002, Shapley et al. 2003, Weiner et al. 2009, Chisholm et al. 2017b). Consequently, we used the non-parametric approach described in Gazagnes et al. (2018) to measure the H I covering fraction from the residual flux of the Lyman series lines after removing the stellar continuum. This method does not presume a specific line profile or velocity distribution of the H I gas. However, this assumes that the $\mathrm{H}_{\mathrm{I}}$ absorption lines are saturated, i.e that $N_{\mathrm{H} \mathrm{I}} \gtrsim 10^{16} \mathrm{~cm}^{-2}$ for the Lyman series lines that we fit (Ly $\beta$ to Ly6). In Gazagnes et al. (2018), we found evidence that the latter assumption is true for 13 galaxies which have $N_{\mathrm{H}_{\mathrm{I}}}$ values $>10^{18.4} \mathrm{~cm}^{-2}$ using the observed $N_{\mathrm{O}_{\text {I }}}$ and the gas-phase metallicity. In the 9 remaining galaxies, we did not find a reliable measurement of $N_{\mathrm{O}_{\mathrm{I}}}$ (for J0925+1409 and the 6 new leakers included from Izotov et al. 2018a b), or the galaxy metallicity has not been measured (SGAS J1226, SGAS J1527). Nevertheless, despite their different oscillator strengths, we observed a tendency for the observed Lyman series to have similar depths and shapes. This is illustrated in Fig. 1 where the Ly $\delta$ and Ly5 absorption lines are plotted in velocity space for $\mathrm{J} 1256+4509$. Similar depths and widths are robust indicators of saturated lines. No H I absorption lines are detected in J1243+4646, the highest LyC escape fraction (see Fig. A.4), likely due to either a very low $\mathrm{H}$ i neutral gas column density or covering fraction.

To measure the covering fraction from the residual flux, we used a Monte-Carlo approach: the observed flux is first divided 


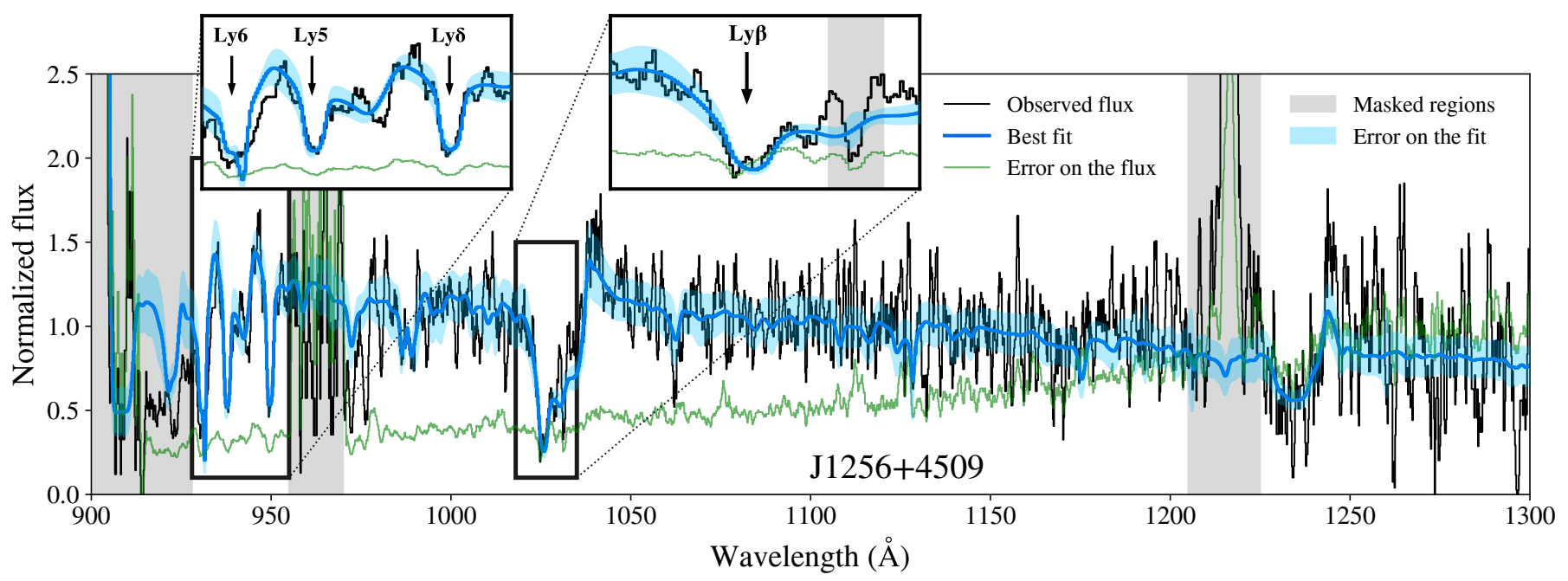

Fig. 2. Best fit (blue solid line) obtained for the galaxy J1256+4509. The black and green solid lines show the observed flux and the error on the flux, respectively. The top panels are zooms on the individual Lyman series lines fitted. The gray shaded areas are the principal wavelength regions masked during the fit, due to geo-coronal emission, low S/N or Ly $\alpha$ emission. We additionally show in the top panels the masks for ISM/Milky Way absorption lines that are not included during the fitting procedure. For display purposes, these masks do not appear in the main panel. The blue shaded area represents the uncertainty on the fit, derived using a Monte-Carlo approach (see details in Sect. 3.1).

by the stellar continuum (fit as $F^{\star}$ in Eq. 1), and modified by a Gaussian kernel centered on zero with standard deviation corresponding to the error array. $C_{f}\left(\mathrm{H}_{\mathrm{I}}\right)$ is derived from the median of 1 minus the residual flux in a velocity range that includes the deepest part of the absorption line. We repeated this procedure 1000 times and took the median and standard deviation of this distribution as the $C_{f}$ value and uncertainty for each $\mathrm{H}_{\text {I }}$ absorption line that is not polluted by Milky Way absorption lines or geocoronal emission. We additionally include the systematic errors in quadrature. We then obtain a final covering fraction by taking the error weighted mean of the $i$ observed Lyman series transitions. Table B.1 lists the $C_{f}$ derived from the residual flux of each Lyman series transition in each galaxy, the resulting $C_{f}\left(\mathrm{H}_{\mathrm{I}}\right)$ "Depth" and the measurement derived from the fitting method. The last column shows the final H i covering fraction, derived from the error weighted mean between the values obtained from the two different approaches. For J1243+4646, which has no detected Lyman series, we still measure the median residual flux in a velocity range chosen where the flux is minimal. We consider the final value as an upper limit. We do not report a $C_{f}\left(\mathrm{H}_{\mathrm{I}}\right)$ "Depth" for GP0303-0759 because its only $\mathrm{H}$ I absorption line observed is contaminated by a Milky Way absorption line.

We note that the galaxies in our sample have a different number of observed Lyman Series lines. However, for galaxies with more than one observed $\mathrm{H}_{\mathrm{I}}$ absorption line, the individual $C_{f}\left(\mathrm{H}_{\mathrm{I}}\right)$ estimates using the depth of the absorption profiles are consistent at $\pm 1 \sigma$ with the value derived when all the lines are fitted simultaneously. Hence, we assume that the Hi parameter values derived in galaxies with a single Lyman series line does not suffer from significant systematic effects. Additionally, Table B.1 shows that both approaches give comparable estimates and uncertainties, thus supporting the fact that both are robust techniques to measure the $\mathrm{H}_{\mathrm{I}}$ covering fraction from saturated Lyman series.

Finally, using the same methodology, we measured the velocity width of each $\mathrm{H}_{\mathrm{I}}$ absorption line, when it is not contaminated by foregrounds or Milky Way absorption, and has sufficient $\mathrm{S} / \mathrm{N}$ so that the line profile is clearly observed. We estimate the velocity width as the velocity range where the absorption profile is at its maximum depth. The minimal and maximal velocities of this interval are chosen where the flux deviates by more than $20 \%$ from the residual flux measurements reported in Table B.1. The same Monte-Carlo approach is used to derive the resulting $v^{\text {width }}$ value and uncertainty for each absorption line, and we obtain the final $v_{\mathrm{HI}}^{\text {widh }}$ for each galaxy derived from the error weighted mean of the individual $v^{\text {width }}$ (last column of Table B.2.

The final covering fraction values, as well as the velocity shift of the line $v_{\mathrm{HI}_{\mathrm{I}}}^{\text {shit }}$, the dust extinction $E_{\mathrm{B}-\mathrm{V}}$ obtained from the fitting method, and average velocity width of the Lyman series are reported in the Table 2. Note that in this work, the synthetic spectra used for the fitting are slightly different from Gazagnes et al. (2018) where the final spectra were only 10 single-age stellar populations of a single metallicity and did not include the nebular continuum. Therefore, we re-measured all the properties in all the galaxies in the sample. While the covering fractions and velocity shift measurements all remained consistent at $\pm 1 \sigma$, we find some small variations in the dust extinction (0.01 to 0.06$)$ for 8 galaxies (marked with $*$ in Table 2). This is expected because the incorporation of nebular continuum and the combination of 5 different metallicities can change the final fitted stellar spectral shape and therefore impact the $E_{\mathrm{B}-\mathrm{V}}$ inferred (see Sect 3.2 ). Overall, the variations are relatively small and do not impact the results obtained in Gazagnes et al. (2018) and Chisholm et al. (2018).

\subsection{Constraining the dust extinction}

Accurately fitting the dust attenuation in the ISM of the galaxies is important to constrain its impact on the escaping radiation. Indeed, several dust models, and/or dust attenuation laws might lead to various physical interpretations of the impact of dust on the Ly $\alpha$ or LyC radiations. Gazagnes et al. (2018) carefully discussed the effects of dust models with a uniform dust screen (all photons are homogeneously attenuated) or with dust free holes (dust only lies in optically thick neutral regions). It was shown that these models result in different values of $C_{f}\left(\mathrm{HI}_{\mathrm{I}}\right)$ and $E_{\mathrm{B}-\mathrm{V}}$, 
Table 3. Investigating the impact of different dust extinction assumptions in $\mathrm{J} 1154+2443$ and $\mathrm{J} 1256+4509$.

\begin{tabular}{|c|c|c|c|c|c|c|c|c|}
\hline Galaxy name & $\begin{array}{l}f_{\mathrm{esc}}^{\mathrm{obs}}(\mathrm{LyC}) \\
(2)\end{array}$ & Law & $E_{\mathrm{B}-\mathrm{V}}$ & $\begin{array}{l}\text { Age } \\
{[\mathrm{Myr}]} \\
(5)\end{array}$ & $C_{f}(\mathrm{H} \mathrm{I})$ & $A(\mathrm{LyC})$ & $A(\operatorname{Ly} \alpha)$ & WSS \\
\hline \multirow{4}{*}{$\mathrm{J} 1154+2443$} & \multirow{4}{*}{0.46} & & $0.118 \pm 0.031$ & 8.10 & $0.401 \pm 0.152$ & 1.51 & 1.20 & 733 \\
\hline & & Reddy +16 & $0.00 *$ & 15.07 & $0.379 \pm 0.145$ & 0.00 & 0.00 & 770 \\
\hline & & & $0.20 *$ & 4.20 & $0.339 \pm 0.183$ & 2.57 & 2.04 & 743 \\
\hline & & $\mathrm{SMC}$ & $0.034 \pm 0.008$ & 7.99 & $0.483 \pm 0.390$ & 0.87 & 0.55 & 733 \\
\hline \multirow{4}{*}{$\mathrm{J} 1256+4509$} & \multirow{4}{*}{0.38} & \multirow{3}{*}{ Reddy +16} & $0.076 \pm 0.029$ & 2.52 & $0.408 \pm 0.125$ & 0.98 & 0.78 & 1517 \\
\hline & & & $0.00 *$ & 3.15 & $0.440 \pm 0.121$ & 0.00 & 0.00 & 1555 \\
\hline & & & $0.20 *$ & 2.32 & $0.571 \pm 0.183$ & 2.57 & 2.04 & 1518 \\
\hline & & SMC & $0.038 \pm 0.008$ & 2.38 & $0.383 \pm 0.133$ & 0.98 & 0.62 & 1499 \\
\hline
\end{tabular}

Notes. (1) Galaxy name; (2) Observed LyC escape fraction; (3) dust attenuation law; (4) dust extinction; (5) light-weighted stellar continuum age from the fit, derived from the linear combination of the 50 single-age STARBURST99 stellar continuum; (6) H i covering fraction value and uncertainty returned by the fit; (7) Attenuation at $912 \AA$; (8) Attenuation at $1216 \AA$ and (9) the value of the summed squared weighted residuals for the returned parameter values as given by MPFIT.

* Values fixed during the fitting procedure.
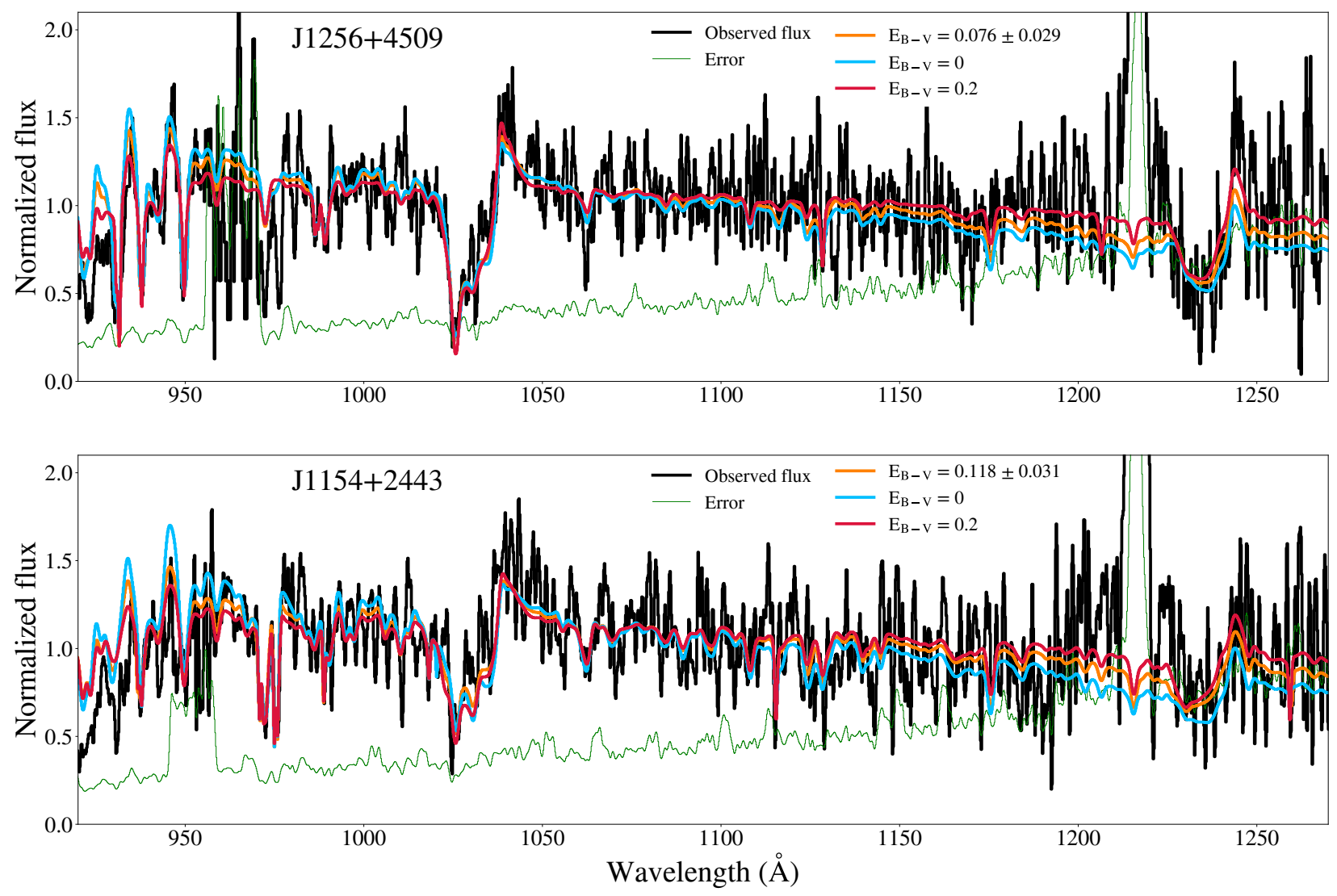

Fig. 3. Top: the black curve is the observed flux in the galaxy $\mathrm{J} 1256+4509$, green is the error, orange is the fit obtained letting $E_{\mathrm{B}-\mathrm{V}}$ as a free parameter, blue and red lines are fits obtained when fixing $E_{\mathrm{B}-\mathrm{V}}$ to 0 and 0.2 respectively. Bottom: same for the galaxy J1154+2443. Both spectra have been normalized using the median flux between 1090 and $1100 \AA$. The wavelength regions with Ly $\alpha$ emission (between 1205 to $1225 \AA$ ) or low $\mathrm{S} / \mathrm{N}(<1)$ are masked during the fitting procedure (see Fig. 2 ).

but lead to very similar estimates of the absolute escape fraction of LyC radiation.

Additionally, the choice of the dust extinction curve significant impacts the effect of dust obscuration on the wavelength region around and below the Lyman break. Typical dust at- tenuation curves (e.g from Calzetti et al. 2000, or the Small Magellanic Cloud (SMC) law) are unconstrained in the far-UV ( $\lambda<1250 \AA$ ), while measuring the impact of dust at these wavelengths is crucial when investigating the escape of ionizing photons from dusty galaxies. In this work, we choose the 
dust extinction law derived in Reddy et al. (2016a). Our choice is motivated by the fact that the authors used a large sample of 933 far-UV observations of Lyman Break Galaxies at $\mathrm{z} \sim 3,121$ having a deep spectroscopic coverage from 850 to $1300 \AA$, to robustly constrain the shape of the dust attenuation curve between 950 and $1500 \AA$. The authors report that the attenuation of LyC photons around $900 \AA$ is $\approx 2$ times lower than estimates derived from polynomial extrapolations of typical dust attenuation curves (Calzetti et al. 2000, Reddy et al. 2015). In Gazagnes et al. (2018), we investigated the effects of using different attenuation laws, such as the SMC attenuation law ${ }^{3}$, which is significantly steeper than the Reddy et al. (2016a) law, and showed that this had a relatively small impact on the derived values of the $\mathrm{H}_{\mathrm{I}}$ covering fractions. In this work, we reconsider the effects of different dust curves using the examples of J1154+2443 and J1256+4509, two of the three largest leakers in our sample, and report in Table 3 the changes seen with respect to the stellar population age, the $C_{f}(\mathrm{HI})$, the dust extinction and attenuation at $912 \AA$ and $1216 \AA$. We also report the value of the summed squared weighted residuals for the recovered parameter values, WSS, as returned by MPFIT, to demonstrate the differences in the quality of the fit in each case.

The age of the stellar population and the $\mathrm{H}$ I covering fraction are insensitive to the dust attenuation law used. This is because the presence of specific stellar features in the spectra fix the stellar populations during the fit (Chisholm et al. 2019). However, we derive approximately two times lower attenuation for the Ly $\alpha$ and LyC radiation in J1154+2445 when using the SMC law. Thus, the choice of the dust law can impact the fitted attenuation, while the differences in the summed squared weighted residuals are too small to choose a dust extinction curve which significantly improves the final fit quality.

Additionally, we further investigated the fluctuations of the parameters inferred when $E_{\mathrm{B}-\mathrm{V}}$ is fixed to 0 and 0.2 for a given dust extinction law. These results are reported in Table 3 and the obtained fits are shown in Fig. 3. $\mathrm{A} \pm 0.2$ difference in $E_{\mathrm{B}-\mathrm{V}}$ leads to small variations (within the flux error) in the shape of the fitted spectra while having a significant impact on the attenuation at 912 and $1216 \AA$, reducing the flux by $85 \%$ in the model with $E_{\mathrm{B}-\mathrm{V}}=0.2$ compared to that in the model with $E_{\mathrm{B}-\mathrm{V}}=$ 0 . However, the differences in the residuals are insignificant for selection of $E_{\mathrm{B}-\mathrm{V}}$. This is likely because there is a strong degeneracy between the stellar population age and the dust extinction needed to model the observed flux. Indeed, younger populations have a steeper continuum towards the far-UV wavelengths, and require a larger dust extinction to reproduce the observed flux compared to older star populations. This is consistent with the results in Table 3, since we obtained younger and older stellar populations when fixing $E_{\mathrm{B}-\mathrm{V}}$ to 0.2 and 0 , respectively. The poorly constrained stellar population age can affect the reliability of $f_{\mathrm{esc}}^{\mathrm{obs}}(\mathrm{LyC})$ when the former is derived using the ratio of the observed flux at $<912 \AA$ over the estimated intrinsic emission of ionizing photons.

Robustly constraining the stellar populations requires to identify specific markers of the presence of young or old star populations. Izotov et al. (2016a b. 2018a b) report that all leak-

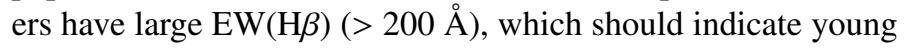
stellar populations (Stasińska \& Leitherer 1996). However, in practice, exact age determinations from $\mathrm{EW}(\mathrm{H} \beta)$ are compli-

\footnotetext{
3 Values have been taken from the IDL routine from J. Xavier Prochaska: https://github.com/profxj/xidl/tree/master/ Dust
}

cated, since it depends on the star formation history and the IMF of the stellar population. Additionally, the strength of the stellar features such as the O vi and N v P-Cygni profiles at 1020$1040 \AA$ and $1220-1240 \AA$ respectively is related to the stellar population properties. However, the relatively low $\mathrm{S} / \mathrm{N}$ of our observations make it challenging to constrain the age of the stellar population. Chisholm et al. (2019) recently emphasized that high $\mathrm{S} / \mathrm{N}$ is required to properly constrain the stellar population of the galaxy, especially to accurately derive the escape of ionizing photons from SED fitting. Therefore, robustly constraining the dust attenuation in our sample would require deeper observations.

Table 3 shows that the fitted $C_{f}(\mathrm{HI})$ is rather insensitive to variations of stellar age or dust extinction, and still provides reliable covering fraction estimate to investigate its impact on the Ly $\alpha$ and LyC escape.

\section{3. $L y \alpha$ properties}

Table 4 reports the Ly $\alpha$ properties for all galaxies in our sample. One galaxy has a Ly $\alpha$ absorption profile, two have Ly $\alpha$ seen both in absorption and emission with a single peak profile. The 19 remaining galaxies have Ly $\alpha$ seen in emission, 18 exhibiting a double peak profile and one having a triple peak profile (J1243+4646). Eighteen of the 22 galaxies had their Ly $\alpha$ profiles studied in the literature (Henry et al. 2015; Izotov et al. 2016a b; Verhamme et al. 2017; Puschnig et al. 2017; Izotov et al. 2018a b; Orlitová et al. 2018). We re-measured the Ly $\alpha$ properties in all spectra to avoid inconsistencies due to different measurement methods. The Ly $\alpha$ escape fractions were recalculated coherently using the equation

$f_{\mathrm{esc}}^{\mathrm{Ly} \alpha}=\frac{\mathrm{F}(\mathrm{Ly} \alpha)}{8.7 \times \mathrm{F}_{\mathrm{corr}}(\mathrm{H} \alpha)}$,

where $\mathrm{F}(\operatorname{Ly} \alpha)$ is the observed $\operatorname{Ly} \alpha$ flux, corrected for the Milky Way extinction, $\mathrm{F}_{\text {corr }}(\mathrm{H} \alpha)$ is the $\mathrm{H} \alpha$ flux corrected for both internal and Milky Way extinction, and 8.7 is the assumed ratio between the intrinsic $\mathrm{Ly} \alpha$ and $\mathrm{H} \alpha$ flux assuming Case-B recombination with a temperature of $10^{4} \mathrm{~K}$ and an electron density of $n_{e}=350 \mathrm{~cm}^{-3}$. We note that Case-B recombination assumes that the gas in the ISM is optically thick to radiation above $13.6 \mathrm{eV}$, thus may not be valid for galaxies where a substantial amount of ionizing photons escape. In galaxies with an optically thin ISM (Case-A recombination), the effective recombination coefficient for the Balmer hydrogen lines can increase by a factor $\sim 1.5$ (Osterbrock 1989). In Izotov et al. (2018a), the authors derived an intrinsic $\mathrm{Ly} \alpha-\mathrm{H} \alpha$ flux ratio of $\approx 11.2$ in the galaxy $\mathrm{J} 1154+2443$ using CLOUDY models (Ferland et al. 2013, 2017). We investigated in Appendix B.3 the impact of using a different intrinsic $\mathrm{F}(\mathrm{Ly} \alpha) / \mathrm{F}(\mathrm{H} \alpha)$ ratio on the observed $f_{\text {esc }}(\mathrm{Ly} \alpha)$ trends derived in this work. Overall, we show that the new significance levels of these trends differ by at most $-0.5 \sigma$, while all the correlations remain significant at least at the $2.5 \sigma$ level. Thus we assume that fluctuations in the intrinsic $\operatorname{Ly} \alpha \mathrm{H} \alpha$ flux ratio should not substantially affect the results presented in this work.

For the low-redshift galaxies, we calculated $f_{\mathrm{esc}}(\mathrm{Ly} \alpha)$ using the $\operatorname{Ly} \alpha$ and $\mathrm{H} \alpha$ flux measurements, already corrected for internal and Milky Way extinction, reported in the reference papers. While some of the sources in our sample have $\operatorname{Ly} \alpha$ emission outside the COS aperture (Henry et al. 2015), we did not apply aperture correction because the size of the extended Ly $\alpha$ emission varies from galaxy to galaxy. The final errors were derived by propagating the errors of the individual measurements. We 
Table 4. Ly $\alpha$ properties.

\begin{tabular}{|c|c|c|c|c|c|c|c|}
\hline Galaxy name & $\frac{\mathrm{F}_{\text {trough }}}{\mathrm{F}_{\text {cont }}}$ & $\begin{array}{l}\operatorname{EW}(\operatorname{Ly} \alpha) \\
{[\AA]} \\
(3)\end{array}$ & $f_{\mathrm{esc}}^{\mathrm{Ly} \alpha}$ & $\begin{array}{l}v_{\mathrm{Ly} \alpha}^{\text {trough }} \\
{\left[\mathrm{km} \mathrm{s}^{-1}\right]} \\
(5)\end{array}$ & $\begin{array}{l}v_{\text {Ly } \alpha}^{\text {blue }} \\
{\left[\mathrm{km} \mathrm{s}^{-1}\right]} \\
(6)\end{array}$ & $\begin{array}{l}v_{\text {Ly } \alpha}^{\text {red }} \\
{\left[\mathrm{km} \mathrm{s}^{-1}\right]} \\
(7)\end{array}$ & $\begin{array}{l}v_{\mathrm{Ly} \alpha}^{\mathrm{sep}} \\
{\left[\mathrm{km} \mathrm{s}^{-1}\right]} \\
(8)\end{array}$ \\
\hline $\mathrm{J} 1243+4646$ & $20.37 \pm 3.58$ & $98 \pm 3$ & $0.52 \pm 0.04$ & $40 \pm 20$ & $-40 \pm 20$ & $130 \pm 40$ & $160 \pm 63$ \\
\hline $\mathrm{J} 1154+2443$ & $11.70 \pm 3.30$ & $135 \pm 4$ & $0.61 \pm 0.03$ & $20 \pm 20$ & $-80 \pm 30$ & $130 \pm 20$ & $240 \pm 36$ \\
\hline $\mathrm{J} 1256+4509$ & $5.83 \pm 2.40$ & $88 \pm 3$ & $0.32 \pm 0.03$ & $10 \pm 40$ & $-90 \pm 50$ & $160 \pm 20$ & $260 \pm 54$ \\
\hline $\mathrm{J} 1152+3400$ & $3.75 \pm 1.01$ & $75 \pm 6$ & $0.34 \pm 0.07$ & $60 \pm 30$ & $-120 \pm 40$ & $190 \pm 30$ & $320 \pm 42$ \\
\hline J1442-0209 & $4.68 \pm 0.72$ & $115 \pm 6$ & $0.54 \pm 0.05$ & $-130 \pm 20$ & $-250 \pm 50$ & $70 \pm 30$ & $320 \pm 58$ \\
\hline J0925+1409 & $3.10 \pm 0.93$ & $80 \pm 5$ & $0.29 \pm 0.03$ & $-30 \pm 30$ & $-160 \pm 20$ & $150 \pm 20$ & $310 \pm 28$ \\
\hline $\mathrm{J} 1011+1947$ & $7.66 \pm 2.04$ & $115 \pm 4$ & $0.18 \pm 0.01$ & $-30 \pm 20$ & $-130 \pm 20$ & $120 \pm 10$ & $260 \pm 22$ \\
\hline $\mathrm{J} 1503+3644$ & $0.95 \pm 0.68$ & $98 \pm 3$ & $0.30 \pm 0.04$ & $-100 \pm 50$ & $-300 \pm 30$ & $140 \pm 30$ & $460 \pm 58$ \\
\hline $\mathrm{J} 1333+6246$ & $1.66 \pm 0.78$ & $73 \pm 2$ & $0.51 \pm 0.09$ & $-150 \pm 20$ & $-300 \pm 40$ & $70 \pm 30$ & $380 \pm 58$ \\
\hline $\mathrm{J} 0901+2119$ & $5.30 \pm 2.40$ & $170 \pm 4$ & $0.14 \pm 0.01$ & $-80 \pm 40$ & $-180 \pm 40$ & $140 \pm 20$ & $320 \pm 45$ \\
\hline $\mathrm{J} 1248+4259$ & $4.85 \pm 2.25$ & $258 \pm 9$ & $0.17 \pm 0.01$ & $-10 \pm 20$ & $-130 \pm 30$ & $140 \pm 30$ & $260 \pm 50$ \\
\hline $\mathrm{J} 0921+4509$ & $0.14 \pm 0.10$ & $5 \pm 3$ & $0.01 \pm 0.01$ & $-220 \pm 50 ; 0 \pm 50$ & $-460 \pm 60$ & $200 \pm 50$ & $660 \pm 92$ \\
\hline Tol1247-232 & $0.08 \pm 0.02$ & $29 \pm 2$ & $0.10 \pm 0.02$ & $-100 \pm 50$ & $-300 \pm 10$ & $150 \pm 10$ & $450 \pm 14$ \\
\hline J0926+4427 & $1.51 \pm 0.10$ & $59 \pm 12$ & $0.20 \pm 0.06$ & $-80 \pm 30$ & $-250 \pm 50$ & $160 \pm 50$ & $410 \pm 71$ \\
\hline $\mathrm{J} 1429+0643$ & $0.82 \pm 0.09$ & $36 \pm 3$ & $0.10 \pm 0.03$ & $20 \pm 30$ & $-220 \pm 50$ & $240 \pm 40$ & $460 \pm 62$ \\
\hline GP0303-0759 & $0.29 \pm 0.20$ & $13 \pm 4$ & $0.05 \pm 0.01$ & $-90 \pm 50$ & $-310 \pm 20$ & $150 \pm 10$ & $460 \pm 22$ \\
\hline GP1244+0216 & $0.23 \pm 0.20$ & $54 \pm 8$ & $0.07 \pm 0.02$ & $-10 \pm 50$ & $-260 \pm 40$ & $250 \pm 20$ & $510 \pm 45$ \\
\hline GP1054+5238 & $0.21 \pm 0.13$ & $14 \pm 3$ & $0.07 \pm 0.02$ & $-10 \pm 60$ & $-220 \pm 50$ & $190 \pm 10$ & $410 \pm 51$ \\
\hline GP0911+1831 & $1.31 \pm 0.34$ & $70 \pm 12$ & $0.16 \pm 0.05$ & $-80 \pm 30$ & $-280 \pm 50$ & $80 \pm 20$ & $360 \pm 64$ \\
\hline SGAS J1226 & $<0.05$ & $-2 \pm 1$ & $<0.01$ & $-120 \pm 40$ & - & $95 \pm 10$ & - \\
\hline SGAS J1527 & $0.07 \pm 0.21$ & $18 \pm 1$ & $<0.01$ & $-160 \pm 20$ & - & $140 \pm 20$ & - \\
\hline Cosmic Eye & $<0.03$ & $-30 \pm 2$ & 0.00 & - & - & - & - \\
\hline
\end{tabular}

Notes. (1) Galaxy name; (2) normalized flux at minimum of the Ly $\alpha$ profile; (3) Ly $\alpha$ restframe equivalent width; (4) Ly $\alpha$ escape fraction; (5) Ly $\alpha$ trough velocity (6) Ly $\alpha$ blue peak velocity; (7) Ly $\alpha$ red peak velocity; and (8) Ly $\alpha$ peak velocity separation. Ly $\alpha$ is seen in absorption in the Cosmic Eye, and only the Ly $\alpha$ red peak is seen in emission in SGAS J1226 and SGAS J1527. J0921+4509 has two troughs in its Ly $\alpha$ profile, see discussion in Section 3.3 The blue and peak velocities reported in Col. (6) and (7) are measured with respect to the systemic velocity. The relative blue and red peak velocities discussed in Sect.4.1 and Fig. 5are obtained by subtracting the trough velocity in Col. (5) from the Cols. (6) and (7), respectively.

derived an Ly $\alpha$ escape fraction of $61 \%$ for $\mathrm{J} 1154+2443$, which is inconsistent with the $98 \%$ reported in Izotov et al. (2018a). However, those authors corrected the Ly $\alpha$ flux with the galaxy internal extinction, which is incompatible with $\mathrm{Eq}(4)$. For the three gravitationally lensed galaxies, we measured $\mathrm{F}(\mathrm{Ly} \alpha)$ using the Monte-Carlo approach described in the Section 3.1. accounting for the lensing magnification factor. The observed flux at Ly $\alpha$ in the Cosmic Eye is consistent with 0, hence we report $f_{\mathrm{esc}}^{\mathrm{Ly} \alpha}=0$. For SGAS J1226 and SGAS J1527, we used the $\mathrm{H} \beta$ flux measurements reported in Wuyts et al. (2012) and assumed an intrinsic $\mathrm{F}(\mathrm{H} \alpha) / \mathrm{F}(\mathrm{H} \beta)$ ratio of 2.85 .

Table 4 reports the Ly $\alpha$ equivalent width, peak and trough velocities, and the normalized flux at the minimum of the profile. These different measurements are illustrated on the Ly $\alpha$ profile of the galaxy J1011+1947 in Fig. 4 All these properties have been measured using the Monte-Carlo method to have consistent values and uncertainties for the entire sample. The Ly $\alpha$ equivalent widths were derived using the observed MW attenuation-corrected spectra, by integrating both the associated Ly $\alpha$ emission and absorption such that the integral limits are chosen, by eye, where the flux meets the stellar continuum. The reported $\operatorname{EW}(\operatorname{Ly} \alpha)$ values are respectively positive and negative for galaxies with a net emission or absorption Ly $\alpha$ profile. The flux above and below the continuum level are respectively The trough velocities $v_{\mathrm{Ly} \alpha}^{\text {trough }}$ are taken as the values when the intensity of the Ly $\alpha$ flux reaches a local minimum. J0921+4509 and $\mathrm{J} 1243+4509$ have peculiar Ly $\alpha$ profiles. The former has two distinct troughs between the red and blue peaks (see Fig. 9), and both their velocities are reported in Table 4. The latter has two peaks bluer than the central trough (see Fig. 7 in Izotov et al. $2018 b$, and we only report the $v_{\mathrm{Ly} \alpha}^{\text {blue }}$ of the peak of maximal intensity. The peak separation, $v_{\mathrm{Ly} \alpha}^{\mathrm{sep}}$, is defined as $v_{\mathrm{Ly} \alpha}^{\text {red }}-v_{\mathrm{Ly} \alpha}^{\text {blue }}$, and the error is derived from the quadratic sum of both uncertainties. Additionally, we measure the flux at the minimum of the Ly $\alpha$ profile as $\frac{F_{\text {trough }}}{F_{\text {cont }}}$, where $F_{\text {trough }}$ is the minimum flux measured at the $\operatorname{Ly} \alpha$ trough, and $\mathrm{F}_{\text {cont }}$ is the median value of the stellar continuum estimated between 1160 and $1270 \AA$, excluding all emission and absorption lines in that range. In this work, we assume that the spectral resolution has a negligible impact on the derived $\frac{\mathrm{F}_{\text {trough }}}{\mathrm{F}_{\text {cont }}}$ values, because the latter was measured in highresolution Ly $\alpha$ spectra $(\mathrm{R} \approx 16000)$. However, the impact of low spectral resolution should be investigated to extend this analysis to samples with lower resolving power. Except for the Ly $\alpha$ escape fraction in $\mathrm{J} 1154+2443$, all the values presented in Table 4 are consistent at $\pm 1 \sigma$ with the previous measurements 


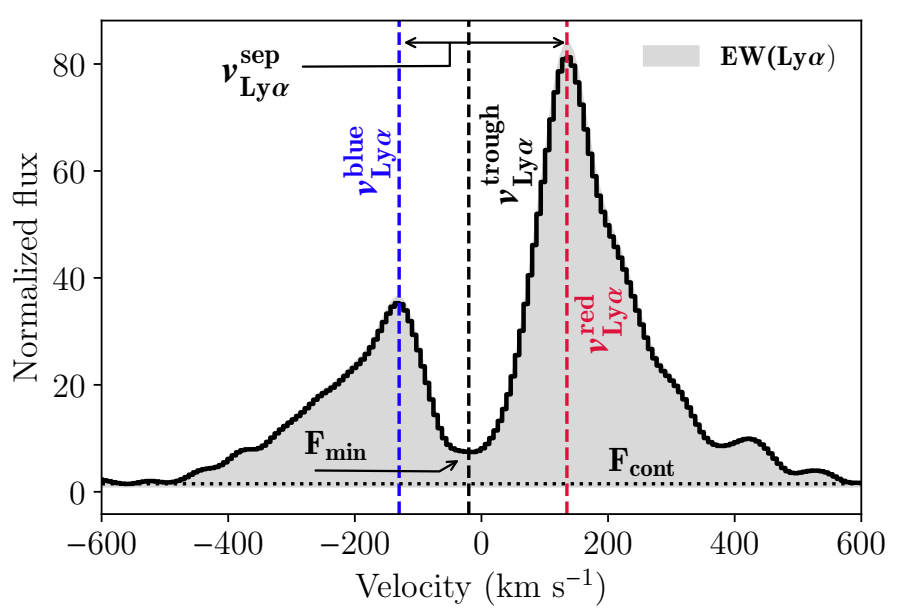

Fig. 4. The Ly $\alpha$ profile observed in the galaxy J1011+1947 with the characteristic measurements annotated. The observed flux has been smoothed by an arbitrary factor for display purposes, and normalized using the median of the flux between 1200 and $1210 \AA$. The gray shaded area represents the integrated region to compute the $\operatorname{Ly} \alpha$ equivalent width.

reported in the literature. Appendix $\mathrm{C}$ shows the Ly $\alpha$ profiles for all galaxies in our sample.

\section{The ISM porosity enables the escape of Ly $\alpha$ and LyC photons}

In this section, we examine the connection between the neutral gas properties, the Ly $\alpha$ properties and the escape of LyC photons.

\subsection{The scattering of $L y \alpha$ photons in a porous ISM}

The Ly $\alpha$ transition is a resonant line and each interaction between Ly $\alpha$ photons and hydrogen atoms shifts the photon's frequency depending on the velocity of the H I gas. Therefore, the emergent Ly $\alpha$ profile provides insights on the neutral hydrogen spatial and velocity distribution. In this section, we investigate the connection between the H I covering fraction and the scattering of Ly $\alpha$ photons, depicted by the blue and red velocity shift of the double peak Ly $\alpha$ profiles. We choose to consider the Ly $\alpha$ emission velocity relative to the $\operatorname{Ly} \alpha$ absorption trough velocity as $v_{\mathrm{Ly} \alpha}^{\text {peak,rel }}=v_{\mathrm{Ly} \alpha}^{\text {peak }}-v_{\mathrm{Ly} \alpha}^{\text {trough }}$. Hence, $v_{\mathrm{Ly} \alpha}^{\text {blue, rel }}$ and $v_{\mathrm{Ly} \alpha}^{\text {red,rel }}$ are derived by subtracting the Col. (5) from the Cols. (6) and (7) in Table 4, respectively. This alternative approach provides insights about the velocity of the last scattering of the blue and red shifted Ly $\alpha$ photons relative to the velocity of the predominant absorption, and also accounts for potential errors in the systemic redshifts (see the discussion in Orlitová et al. 2018). J0921+4509 is a peculiar case with two local minima at different velocities $(-220$ and $0 \mathrm{~km} \mathrm{~s}^{-1}$, see Fig 9). We define its relative peak velocities with respect to the closest absorption troughs, such that $v_{\mathrm{Ly} \alpha}^{\text {blue,rel }}=(-$ $460)-(-220)=-240 \mathrm{~km} \mathrm{~s}^{-1}$ and $v_{\mathrm{Ly} \alpha}^{\text {red,rel }}=(200)-(0)=200 \mathrm{~km}$ $\mathrm{s}^{-1}$.

Figure 5 shows the relation between the $v_{\text {Ly } \alpha}^{\text {blue,rel }}$ and $v_{\text {Ly } \alpha}^{\text {red,rel }}$ and the neutral gas covering fraction. We find a strong correlation between the blue and red relative velocities of the Ly $\alpha$ photons with respect to the $\mathrm{H}$ I gas covering fraction ( $3 \sigma$ signifi- cance, p-value of 0.0026 and 0.0024 respectively). Additionally, we show in Figure 6 the connection between the Ly $\alpha$ peak velocity separation and the $\mathrm{H}$ I covering fraction. We included the recent analysis of the neutral gas properties of 13 low-z GPs with HST-COS observations (GO-14080, PI Jaskot) studied in McKinney et al. (2019) and Jaskot et al. (2019). In the latter study, the authors measured the $C_{f}$ (Si II) of these galaxies, from which we derived the corresponding $C_{f}\left(\mathrm{H}_{\mathrm{I}}\right)$ using the empirical relation $C_{f}(\mathrm{Si}$ II $)-C_{f}(\mathrm{HI})$ found in Gazagnes et al. (2018). We report a correlation at the 3- $\sigma$ leve ${ }^{4}$ (p-value of 0.0018$)$ between $v_{\text {Ly } \alpha}^{\text {sep }}$ and $1-C_{f}(\mathrm{HI})$. The trends reported in Figs. 5 and 6 indicate that Ly $\alpha$ photons experience fewer scattering events in galaxies having low $\mathrm{H}_{\mathrm{I}}$ covering fraction. Interestingly, this somehow differs from Jaskot et al. (2019) and McKinney et al. (2019) who found a weak correlation between $v_{\mathrm{Ly} \alpha}^{\mathrm{sec}}$ and $C_{f}(\mathrm{Si}$ II). This difference might be explained by the fact that $\mathrm{Si}$ II and $\mathrm{H}$ I may not trace exactly the same gas. The $\mathrm{Si}$ II and $\mathrm{H}_{\text {I }}$ ionization potential are similar, but not identical. Hence, the Si II covering fraction is related to, but not equal to $C_{f}\left(\mathrm{HI}_{\mathrm{I}}\right)$ Reddy et al. 2016b. Gazagnes et al. 2018). Additionally, the Si II covering fractions measurements might be impacted by scattering and fluorescent emission in-filling (Prochaska et al. 2011; Scarlata \& Panagia 2015. Mauerhofer et al. in prep).

The scaling relation between $v_{\mathrm{Ly} \alpha}^{\mathrm{sep}}$ and $C_{f}(\mathrm{HI})$ contrasts with the theoretical Ly $\alpha$ studies of Verhamme et al. (2015) and Dijkstra et al.(2016). The latter radiative transfer simulations showed that $v_{\mathrm{Ly} \alpha}^{\mathrm{sep}}$ should scale with decreasing $N_{\mathrm{HI}}$ in the ISM, but the presence of paths cleared of $\mathrm{H}_{\mathrm{I}}$ gas should imprint a single peak profile at the systemic velocity. Nevertheless, the latter analysis assumes that the escape channels are entirely cleared of gas. Other studies showed that the presence of a clumpy ISM (Gronke et al. 2016) and/or the presence of $\mathrm{H}_{\mathrm{I}}$ residuals in the channels (Kakiichi \& Gronke 2019) could lead to a non-unity neutral gas covering fraction, while imprinting a double peak profile. Using the $\mathrm{O}_{\mathrm{I}}$ column densities and reported metallicities, we find that 13 of 22 galaxies in our sample have $N_{\mathrm{H}_{\mathrm{I}}}$ larger than $10^{18} \mathrm{~cm}^{-2}$, while McKinney et al. (2019) found similar results in their sample using both $\mathrm{O}_{\mathrm{I}}$ and $\mathrm{Si}$ II. Hence, in these galaxies, the porosity of the ISM (low $C_{f}\left(\mathrm{HI}_{\mathrm{I}}\right)$ ) should be physically interpreted as the existence of channels with low column densities in an optically thick $\mathrm{H}_{\mathrm{I}}$ environment. To refer to this bi-modal distribution of H I, we introduce the notations $N_{\mathrm{H}_{\mathrm{I}}}^{\text {channel }}$ and $N_{\mathrm{H} \text { I }}^{\text {cloud }}$ to denote the column densities within the channels and within the clouds, respectively. The scaling relation between $v_{\mathrm{Ly} \alpha}^{\text {sep }}$ and $C_{f}\left(\mathrm{H}_{\mathrm{I}}\right)$ suggests that galaxies that have more of these channels also have lower $N_{\mathrm{H}_{\mathrm{I}}}^{\text {chanel }}$. Hence, the presence of low $C_{f}(\mathrm{HI})$ could indirectly trace the abundance of $\mathrm{H}_{\mathrm{I}}$ in the lowest column density regions of the ISM.

The tight connection between the velocity shift of the peaks of the $\operatorname{Ly} \alpha$ emission and $C_{f}(\mathrm{HI})$ additionally indicates that the porosity of the ISM impacts the shape of the Ly $\alpha$ profile. Indeed, in this work, we investigate the relative Ly $\alpha$ peak velocities which can be interpreted as the velocity shift of the escaping Ly $\alpha$ photons with respect to the velocity of the predominant $\mathrm{H}_{\mathrm{I}}$ absorption (similarly to Orlitová et al. 2018). In the literature, Ly $\alpha$ peak velocities are usually considered with respect to the systemic velocity. Interestingly, we do not find any correlation between the red peak velocity relative to the systemic velocity

4 In all this work, for galaxies only having upper limit on $C_{f}\left(\mathrm{H}_{\mathrm{I}}\right)$, we adopt the upper limit when deriving the significance level of the $C_{f}\left(\mathrm{H}_{\mathrm{I}}\right)$ correlations. In Appendix B.3 we investigate how the significance level of the reported trends changes for different assumptions, such as fixing $C_{f}\left(\mathrm{H}_{\mathrm{I}}\right)$ to the lower limit $(0)$, or excluding one or several observations. 

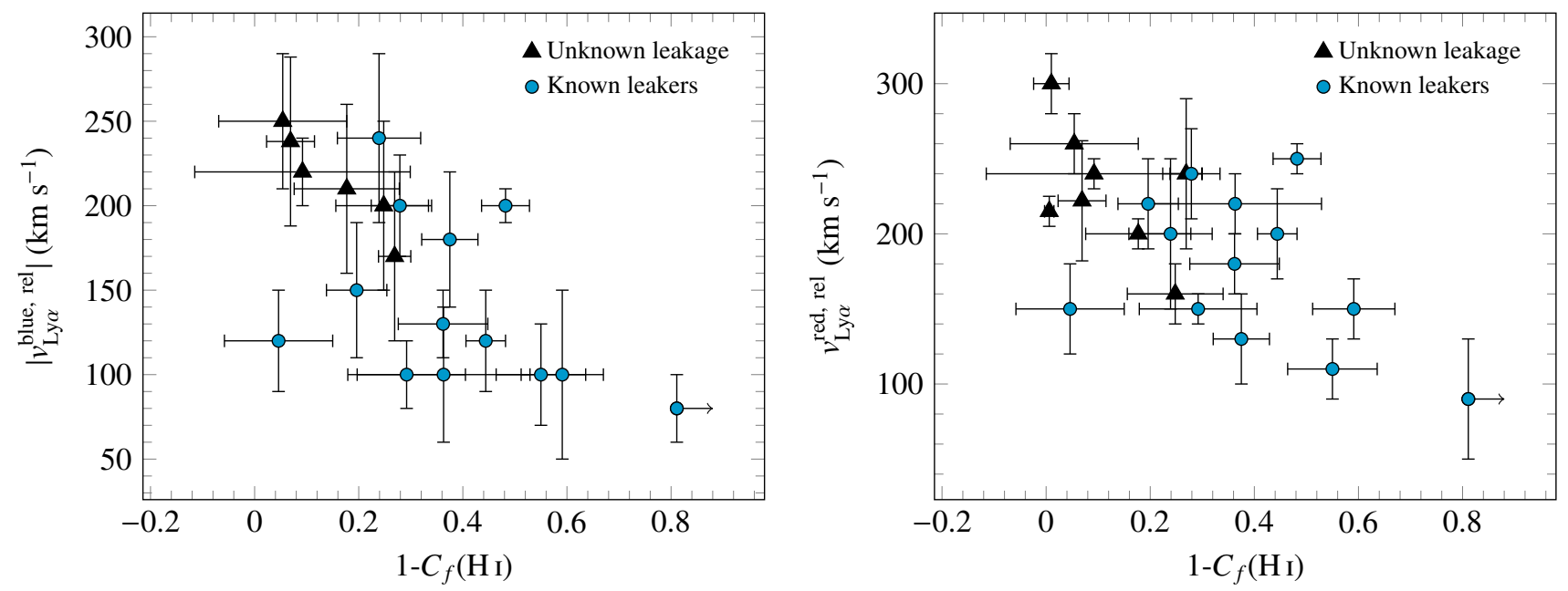

Fig. 5. Left: relation between the relative Ly $\alpha$ blue peak velocity $\left(\left|v_{\mathrm{Ly} \alpha}^{\text {bluerel }}\right|=\left|v_{\mathrm{Ly} \alpha}^{\text {blue }}-v_{\mathrm{Ly} \alpha}^{\text {trough }}\right|\right)$ and 1- $C_{f}\left(\mathrm{H}_{\mathrm{I}}\right)$. Right: relation between the relative Ly $\alpha$ red peak velocity $\left(v_{\mathrm{Ly} \alpha}^{\text {red,rel }}=v_{\mathrm{Ly} \alpha}^{\text {red }}-v_{\mathrm{Ly} \alpha}^{\text {trough }}\right)$ and 1- $C_{f}\left(\mathrm{HI}_{\mathrm{I}}\right)$. Our sample is represented by black triangles and blue circles for galaxies with unknown and known leakage, respectively. J0921+4509 has two main troughs, and we defined its relative peak velocities with respect to the respective closest local trough (see text in Sect. 4.1).

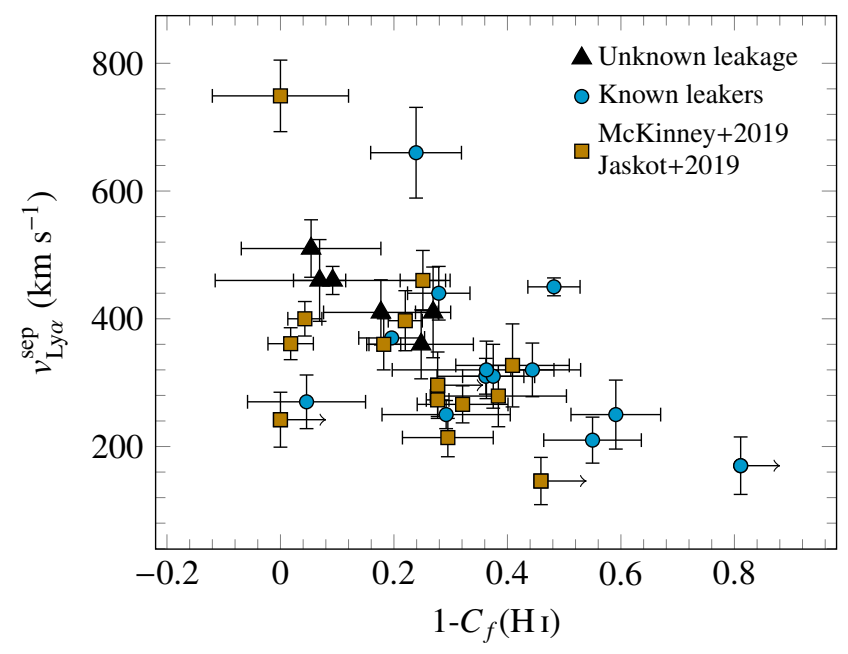

Fig. 6. Relation between the Ly $\alpha$ peak velocity separation $\left(v_{\mathrm{Ly} \alpha}^{\mathrm{sep}}\right)$ versus $1-C_{f}\left(\mathrm{H}_{\mathrm{I}}\right)$. Our sample is represented by black triangles and blue circles for galaxies with unknown and known leakage, respectively. The sample from McKinney et al. (2019) and Jaskot et al. (2019) is shown with orange squares. Note that the latter studies only report $C_{f}(\mathrm{Si}$ II $)$ measurements, from which we derived the corresponding $C_{f}\left(\mathrm{H}_{\mathrm{I}}\right)$ using the empirical $C_{f}\left(\mathrm{Si}\right.$ II)- $C_{f}\left(\mathrm{H}_{\mathrm{I}}\right)$ relation found in Gazagnes et al. (2018).

and the $\mathrm{H}$ r covering fraction and the significance level of the correlation between the blue peak velocity relative to the systemic velocity and $C_{f}(\mathrm{HI})$ is lower $(2 \sigma)$. This suggests that the shift of both the blue and red-shifted Ly $\alpha$ photons relates to the properties of the main $\mathrm{H}_{\mathrm{I}}$ absorption, which traces the bulk of the $\mathrm{H}_{\mathrm{I}}$ gas along the line of sight (LOS). The velocity of the last scattering of Ly $\alpha$ photons likely correlates with the velocity coverage of the thick Hi gas, and with the presence of low-density channels in the ISM. This trend is somewhat surprising for the red Ly $\alpha$ emission. Indeed, the red peak of the Ly $\alpha$ profile corresponds to the back-scattered Ly $\alpha$ photons, which were first emitted in the direction opposite to the observer. In theory, they probe the properties of the gas in the back side of the galaxy ISM, and are expected to have a weaker connection to LOS-dependent properties, such as $C_{f}(\mathrm{HI})$, than blue-shifted photons. Figure 5 shows that this is not the case. This could be because galaxies that have lower $C_{f}(\mathrm{HI})$ also have lower H I column densities in the ISM, such that $v_{\mathrm{Ly} \alpha}^{\text {red,el }}$ indirectly probes the average $N_{\mathrm{H}_{\mathrm{I}}}$ of the neutral gas.

The strong correlation between the peak velocities and the presence of low column density channels could in principle be used to indirectly detect the leakage of Ly $\alpha$ and LyC photons through these sightlines. Kakiichi \& Gronke (2019) suggested that, in an ionization-bounded ISM, the Ly $\alpha$ peak velocity separation could probe $N_{\mathrm{H} \text { I }}^{\text {channel }}$. A similar idea was discussed in Jaskot et al. (2019) where the authors proposed $v_{\mathrm{Ly} \alpha}^{\mathrm{sep}}$ as a probe of the residual $N_{\mathrm{H}_{\mathrm{I}}}$ along the paths of "least resistance" through which Ly $\alpha$ photons escape. These assumptions would explain the strong correlations between low peak velocity separations and $f_{\text {esc }}^{\text {obs }}$ (LyC) (Verhamme et al. 2017; Izotov et al. 2018b, 2020). Nevertheless, we further show in Sect. 4.2 that both low and high column densities of gas impact the $\operatorname{Ly} \alpha$ peak separation, such that a single parameter does not fully determine $f_{\mathrm{esc}}(\mathrm{LyC})$.

\subsection{Narrower $\mathrm{H}$ । absorption lines lead to larger observed Lyo emission}

Because the $\operatorname{Ly} \alpha$ is a resonant transition, the $\operatorname{Ly} \alpha$ photons are strongly impacted by the neutral gas kinematics. Rivera-Thorsen et al. (2015) outlined the role of the velocity coverage of the $\mathrm{H}_{\mathrm{I}}$ gas as an important factor governing the observed emission of Ly $\alpha$ photons. In Fig. 7, we compare the Ly $\alpha$ equivalent width (left panel) and the Ly $\alpha$ escape fraction (right panel) with the $\mathrm{H}$ I velocity width of the Lyman series in our sample. The latter is defined as the velocity interval where the $\mathrm{H}_{\mathrm{I}}$ absorption profile is at its maximum depth. We find strong anti-correlations both with $\operatorname{EW}(\operatorname{Ly} \alpha)(4 \sigma, \mathrm{p}$-value of 0.000025$)$ and with $f_{\mathrm{esc}}(\operatorname{Ly} \alpha)(3$ $\sigma$, p-value of 0.0007$)$ that confirm that the velocity width of the optically thick $\mathrm{H}_{\mathrm{I}}$ absorption significantly impacts the emission and escape of Ly $\alpha$ photons. These results suggest that fewer Ly $\alpha$ photons escape from galaxies with broad $\mathrm{H}_{\mathrm{I}}$ absorption lines, be- 

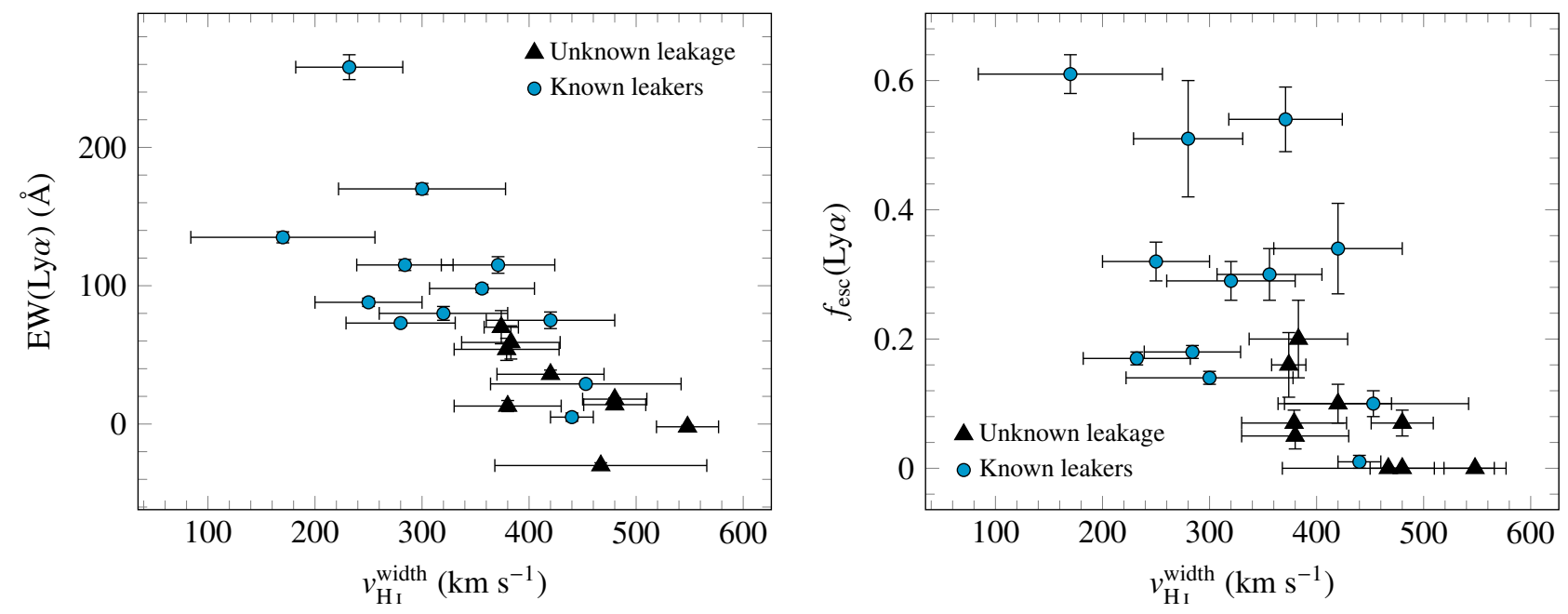

Fig. 7. Ly $\alpha$ equivalent width (left) and escape fraction (right) versus the width of the $\mathrm{H}_{\mathrm{I}}$ maximal absorption. Galaxies with a lower velocity width of the Lyman series have higher $\operatorname{EW}(\operatorname{Ly} \alpha)$ and $f_{\text {esc }}(\operatorname{Ly} \alpha)$.

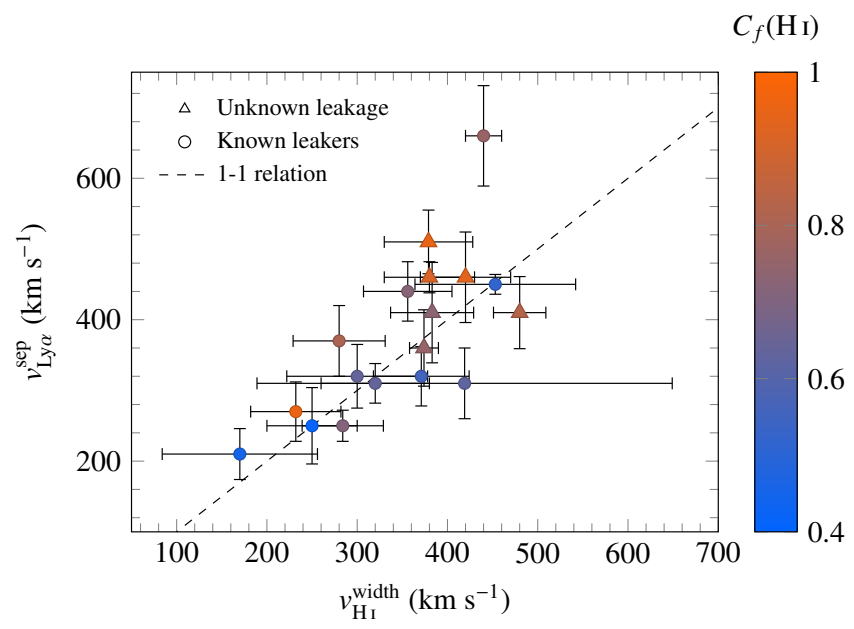

Fig. 8. Ly $\alpha$ peak velocity separation versus the H i velocity width of maximal absorption. The color bar represents the $\mathrm{H}_{\mathrm{I}}$ covering fraction measurement of each galaxy. Overall, the Ly $\alpha$ peak velocity separation scales with the width of the maximal absorption of the $\mathrm{H}_{\mathrm{I}}$ gas, and galaxies with larger $C_{f}\left(\mathrm{H}_{\mathrm{I}}\right)$ have larger $v_{\mathrm{Ly} \alpha}^{\text {sep }}$ than $v_{\mathrm{HI}}^{\text {widh }}$, such that the Ly $\alpha$ emission peaks at velocities outside of the $\mathrm{H}_{\mathrm{I}}$ absorption.

cause Ly $\alpha$ photons are more likely to encounter optically thick neutral gas and hence have a higher probability to be destroyed by dust grains. However, disentangling the physical origin of a large $v_{\mathrm{H}_{\mathrm{I}}}^{\text {widh }}$ is not trivial. This can either result from large $N_{\mathrm{H}}$, or from a broad velocity range of the absorbing gas. Both cases should similarly lower the escape and observed emission of Ly $\alpha$ photons because for Ly $\alpha$ photons to escape, it needs to be scattered to velocities where the gas is transparent. Nevertheless, this is different for LyC photons, since they are sensitive to the $N_{\mathrm{H}_{\mathrm{I}}}$ in the ISM, but not to the kinematics of the H I gas.

Fig. 8 provides additional insights about the origin and impact of broad $\mathrm{H}_{\mathrm{I}}$ absorption lines on the escape of $\mathrm{Ly} \alpha$ and $\mathrm{LyC}$ photons. It shows the linear relation (at the $3 \sigma$ significance, p-value of 0.0007 ) between the $\mathrm{H}_{\mathrm{I}}$ velocity width of the maximal absorption and the Ly $\alpha$ peak velocity separation. $v_{\mathrm{Ly} \alpha}^{\text {sep }}$ is linearly consistent at $\pm 1 \sigma$ with $v_{\mathrm{HI}_{\mathrm{I}}}^{\text {width }}$ for 17 galaxies $(\sim 80 \%)$ in our sample. Because $v_{\mathrm{Ly} \alpha}^{\text {sep }}$ scales with $N_{\mathrm{H} \text { I }}$ (Verhamme et al.
2015, Dijkstra et al. 2016, this trend could suggest that the observed width of the $\mathrm{H}_{\mathrm{I}}$ absorption lines indirectly probes the $\mathrm{H}$ I column densities in the dense regions $\left(N_{\mathrm{HI}}^{\text {cloud }}\right)$ which are imprinted from the saturated Lyman series. Additionally, recent observational studies have highlighted the connection between low peak velocity separation and the escape of ionizing photons (Verhamme et al. 2017; Izotov et al. 2018b, 2020). Interestingly, we find that J1154+2443 and J1256+4509, the two largest leakers (38 and 46\%) with Lyman series observations, have the lowest $v_{\mathrm{HI}_{\mathrm{I}}}^{\text {width }}$ in our sample. Similarly, we do not detect $\mathrm{H}_{\mathrm{I}}$ in the largest $f_{\text {esc }}^{\text {obs }}$ (LyC) LCE (J1243+4646). Hence, in these galaxies, the thick neutral clouds in the ISM might have $N_{\mathrm{H}_{\mathrm{I}}}^{\text {cloud }}$ low enough to let a significant fraction of ionizing photons escape. Note that this hypothesis is not incompatible with the presence of saturated $\mathrm{H}_{\mathrm{I}}$ absorption lines, because the range of Lyman series lines we study in this work ( $\mathrm{Ly} \beta$ to Ly6) saturates at $N_{\mathrm{H}}$ larger than $\sim 10^{16} \mathrm{~cm}^{-2}$. Additionally, we do not report any significant trend between $f_{\mathrm{esc}}^{\text {obs }}(\mathrm{LyC})$ and $v_{\mathrm{H}_{\mathrm{I}}}^{\text {width }}$ in the low LyC leakers $\left(f_{\mathrm{esc}}^{\mathrm{obs}}(\mathrm{LyC})<13 \%\right)$. Hence, for these galaxies, the $N_{\mathrm{H}_{\mathrm{I}}}^{\text {cloud }}$ is likely too large and efficiently absorbs all the ionizing radiation that passes through the clouds. This is consistent with Gazagnes et al. (2018) where we estimated $N_{\mathrm{H}_{\mathrm{I}}}$ larger than $10^{18} \mathrm{~cm}^{-2}$ using $N_{\text {OI }}$ for 6 of the 10 low LyC leakers with observed O I absorption lines. Hence, this result could emphasize that the leakage of LyC photons in the largest leakers is a combination of ionization and density bounded mechanisms, highlighted by the presence of both low $C_{f}\left(\mathrm{H}_{\mathrm{I}}\right)$ and low $v_{\mathrm{HI}}^{\text {width }}$. However, this outcome should be taken with caution, because $v_{\mathrm{H}_{\mathrm{I}}}^{\text {width }}$ is highly degenerate with the velocity distribution of the Hi gas. We discuss further this point in Sect. 4.5 .

In Sect. 4.1, we showed the tight correlation between $v_{\text {Ly } \alpha}^{\text {sep }}$ and the $\mathrm{H}$ i covering fraction. Fig. 8 additionally shows the impact of low $C_{f}\left(\mathrm{H}_{\mathrm{I}}\right)$ on the relation between $v_{\mathrm{Ly} \alpha}^{\text {sep }}$ and $v_{\mathrm{H}_{\mathrm{I}}}^{\text {width }}$. Overall, galaxies with large $C_{f}(\mathrm{HI})$ have $v_{\mathrm{Ly} \alpha}^{\text {sep }}$ larger than $v_{\mathrm{H}_{\mathrm{I}}}^{\text {width }}$, such that the Ly $\alpha$ emission peaks at velocities beyond the H I absorption. This is illustrated in the left panel of Fig.9, where we superpose the $\mathrm{Ly} \alpha$ profile and the $\mathrm{Ly} \beta$ line for the galaxy $\mathrm{J} 0921+4509$. $\mathrm{J} 0921+4509$ has a relatively high covering fraction, $\left(C_{f}\left(\mathrm{H}_{\mathrm{I}}\right) \approx\right.$ $0.8)$ and the peaks of its Ly $\alpha$ emission are located on the edges of the optically thick $\mathrm{H}_{\mathrm{I}}$ absorption. On the other hand, Fig. 8 shows that galaxies with a lower $C_{f}\left(\mathrm{H}_{\mathrm{I}}\right)$ have $v_{\mathrm{Ly} \alpha}^{\text {sep }} \leq v_{\mathrm{HI}}^{\text {width }}$. This 

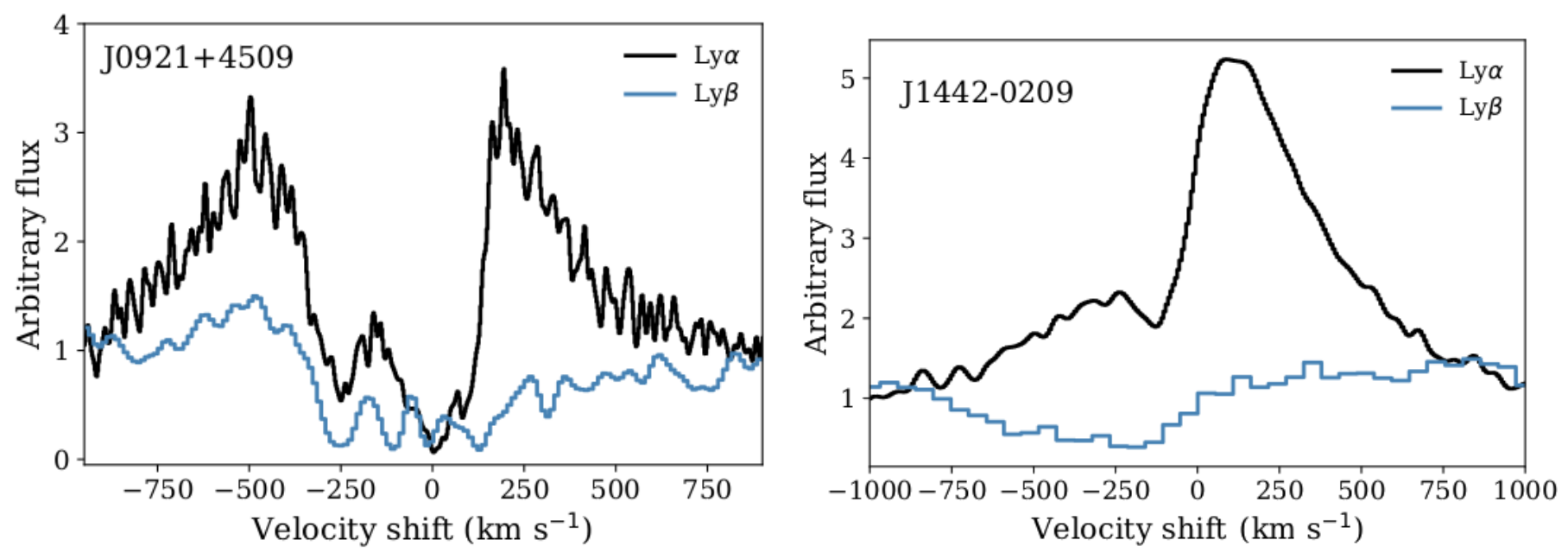

Fig. 9. Left: plot of the $\mathrm{Ly} \alpha$ emission line and $\mathrm{Ly} \beta$ absorption line in $\mathrm{J} 0921+4509\left(C_{f}\left(\mathrm{H}_{\mathrm{I}}\right)=0.761\right)$. Right: plot of the $\mathrm{Ly} \alpha$ emission line and $\mathrm{Ly} \beta$ absorption line in $\mathrm{J} 1442-0209\left(C_{f}\left(\mathrm{H}_{\mathrm{I}}\right)=0.556\right)$. The Ly $\alpha$ spectra have been smoothed to half the resolution of the observations, and scaled down with a power law for display purposes. The presence of Ly $\alpha$ emission on top of the optically thick H I absorption line suggests that Ly $\alpha$ photons can escape at lower velocities through low-density channels. Similar plots for all the galaxies in our sample are in the Appendix C

is because decreasing $C_{f}(\mathrm{HI})$ increases the probability of Ly $\alpha$ photons to find an escape road through low-density channels and thus lowers the amount of scattering events for these photons (see Sect. 4.1). The right panel of Fig. 9 shows the superposition of Ly $\alpha$ and $\operatorname{Ly} \beta$ for J1442-0209 which has a lower $C_{f}\left(\mathrm{H}_{\mathrm{I}}\right)$ $(\approx 0.6)$. The blue peak falls directly on top of the optically thick part of the $\mathrm{Ly} \beta$ absorption line, which implies that a dominant proportion of $\operatorname{Ly} \alpha$ photons on the blue portion of the line escape at velocities where the neutral gas is optically thick. Thus, the presence of Ly $\alpha$ emission on the top of the H I absorption is evidence of the existence of low-density channels (low $C_{f}(\mathrm{HI})$ ), and strongly indicates whether Ly $\alpha$ photons are able to escape from the galaxy. This also supports a plausible origin for the role of the blue Ly $\alpha$ peak in identifying the LyC escape (Henry et al. 2015; Orlitová et al. 2018), because LyC photons should escape through the same low-density paths along the line of sight.

In Sect. 4.1, we found a tight correlation between $v_{\mathrm{Ly} \alpha}^{\text {red,rel }}$ and $C_{f}(\mathrm{HI})$ indicating that the back-scattered emission is similarly affected by the presence of low-density channels. Nevertheless, Fig. 9 shows that the red emission peaks at velocities outside of the optically thick $\mathrm{H}_{\mathrm{I}}$ absorption. More generally, we found that this is the case for all galaxies in our sample (Appendix C shows the combinations of the Ly $\alpha$ and Lyman series profiles). In Sect. 4.1, we suggested that the relation between the velocity shift of the red peak of the Ly $\alpha$ profile and $C_{f}\left(\mathrm{H}_{\mathrm{I}}\right)$ could arise from the presence of both lower $N_{\mathrm{HI}}^{\text {channel }}$ and lower $N_{\mathrm{HI}}^{\text {cloud }}$ in the galaxies with the lowest neutral gas covering fraction. Hence, this may suggest that there exists a connection between low $\mathrm{H}_{\mathrm{I}}$ velocity width of maximal absorption (related to $N_{\mathrm{H} \text { I }}^{\text {cloud }}$ ) and low $\mathrm{H}$ I covering fraction (related to $N_{\mathrm{H}_{\mathrm{I}}}^{\text {chanel }}$ ). We report a moderate 2- $\sigma$ correlation between $C_{f}\left(\mathrm{H}_{\mathrm{I}}\right)$ and $v_{\mathrm{HI}}^{\text {width }}$, hence, we can not significantly conclude that such relation exists.

Overall, the strong correlations between the peak velocity separation and the neutral gas covering fraction (Sect. 4.1), or the width of the saturated Lyman series, suggests that $v_{\mathrm{Ly} \alpha}^{\mathrm{sep}}$ is sensitive both to the presence of low column density paths and to the properties of the dense H I regions in the ISM. As mentioned above, this might be because the blue peak is more sensitive to the existence of channels through which Ly $\alpha$ and LyC photons more easily escape, while the red peak probes the overall abun- dance of $N_{\mathrm{H}_{\mathrm{I}}}$ in the ISM. Hence, both lower H $\mathrm{H}_{\mathrm{I}}$ covering fraction, and lower $N_{\mathrm{H} \text { I }}^{\text {cloud }}$ similarly impact the peak separation of the Ly $\alpha$ profile because the latter is sensitive to the "total" H I properties of the ISM of galaxies. While this outcome is somewhat surprising, it suggests that the peak velocity separation is a robust probe of the presence of low column density paths towards the observer, and a key observable to investigate the escape of Ly $\alpha$ or LyC photons in low redshift LCE candidates (Verhamme et al. 2017, Izotov et al. 2018b, 2020). Nevertheless, these outcomes also suggest that measuring a low $v_{\mathrm{Ly} \alpha}^{\mathrm{sep}}$ is not enough to disentangle the dominant leakage mechanisms, because both the ISM porosity and the width of the saturated $\mathrm{H}$ I absorption lines impact the peak separation velocity. We discuss this point further in Sect.5.1.

At higher redshift, where observing the Lyman Series is highly unlikely because of the presence of line-of-sight neutral gas in the IGM, the ISM porosity can be traced with $C_{f}(\mathrm{Si}$ II), which probes the presence of low-density channels when the Lyman series is not observable (Gazagnes et al. 2018). Additionally, Chisholm et al. (2016) found tight correlations between the velocity widths of different ionic transitions, and suggest that the velocity width of $\mathrm{Si}$ II is related to $v_{\mathrm{HI}}^{\text {widh }}$. The combination of both could be used to estimate the $\operatorname{Ly} \alpha$ peak separation and probe the Ly $\alpha$ and LyC escape of photons at higher redshift. We discuss this further in Sect. 5.2

\subsection{The Lyo equivalent width scales with low $C_{f}\left(\mathrm{H}_{\mathrm{I}}\right)$}

The $\operatorname{EW}(\operatorname{Ly} \alpha)$ and neutral gas properties of Lyman $\alpha$ Emitters have been investigated in numerous studies (Rivera-Thorsen et al. 2015; Verhamme et al. 2017, Chisholm et al. 2017a; Steidel et al. 2018; Orlitová et al. 2018; Du et al. 2018; McKinney et al. 2019, Trainor et al. 2019; Jaskot et al. 2019; Runnholm et al. 2020). Figure 10 explores the connection between the $\mathrm{EW}(\operatorname{Ly} \alpha)$, the neutral gas coverage and the velocity width of the maximal absorption of the $\mathrm{H}_{\mathrm{I}}$ lines. Jaskot et al. (2019) found a positive correlation between the escape of Ly $\alpha$ photons and the Si II covering fraction in highly ionized GPs. Their sample, as well as $\mathrm{J} 1243+4646$, appear in grey in Fig. 10 as they do not have measured $v_{\mathrm{H}_{\mathrm{I}}}^{\text {widh }}$. We report a $2.5 \sigma$ significance relation (p-value of 


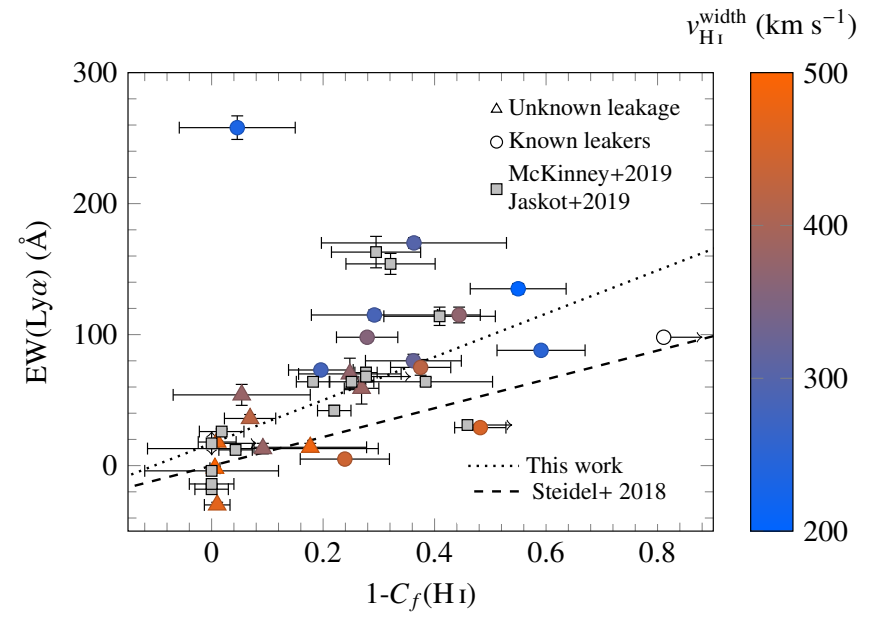

Fig. 10. Ly $\alpha$ equivalent width versus the porosity of the neutral gas as given by $1-C_{f}\left(\mathrm{H}_{\mathrm{I}}\right)$. The color bar represents the $\mathrm{H}_{\mathrm{I}}$ velocity interval where the Lyman series is at its maximum depth. Our sample is represented by triangles and circles for galaxies with unknown and known leakage respectively, and the sample from McKinney et al. (2019) and Jaskot et al. (2019) is shown with gray squares as they do not have $\mathrm{H}_{\mathrm{I}}$ velocity width measurements. The dashed line shows the empirical relation found in Steidel et al. (2018), while the dotted line is the fit to the data, excluding J1248+4259 (see Eq (5). This figure suggests that Ly $\alpha$ emission strongly scales with the covering fraction of the channels and with the width of the saturated $\mathrm{H}_{\mathrm{I}}$ absorption lines.

$0.004)$, between $\mathrm{EW}(\mathrm{Ly} \alpha)$ and $1-C_{f}(\mathrm{HI})$. The trend is scattered, and Fig. 10 shows that most galaxies with high $\operatorname{EW}(\operatorname{Ly} \alpha)$ have both low $C_{f}\left(\mathrm{HI}_{\mathrm{I}}\right)$ and low $v_{\mathrm{HI}}^{\text {width }}$. This supports the picture where both the presence of channels with low $N_{\mathrm{H}_{\mathrm{I}}}^{\text {chanel }}$ and narrower $\mathrm{H}_{\mathrm{I}}$ absorption lines increase the observed emission of Ly $\alpha$ photons. Nevertheless, the interpretation of the Ly $\alpha$ EW is not trivial because $\operatorname{EW}(\operatorname{Ly} \alpha)$ is also degenerate with varying galaxy properties, such as burst age, star formation history or metallicity.

One can note the peculiar case of the galaxy J1248+4259, with the largest $\mathrm{EW}(\mathrm{Ly} \alpha)$ in both samples $(258 \AA)$, but a relatively low $1-C_{f}\left(\mathrm{H}_{\mathrm{I}}\right)$ (0.046). Surprisingly, J1248+4259 has one of the weakest $\mathrm{O}$ vi and $\mathrm{N} v$ P-Cygni profiles in our sample (see Fig. A.4), which suggest the presence of an older population of stars, at first sight incompatible with the presence of large $\mathrm{EW}(\mathrm{Ly} \alpha)$ (Schaerer 2003). On the other hand, weak O vi and N v P-Cygni profiles could be explained by a young stellar population, but without forming the most massive stars. We also report a low $v_{\mathrm{H}_{\mathrm{I}}}^{\text {width }}$ for $\mathrm{J} 1248+4259$, which could partly explain the large $\mathrm{EW}(\mathrm{Ly} \alpha)$ measured. Indeed, as seen in Sect. 4.2 . low $v_{\mathrm{H}_{\mathrm{I}}}^{\text {width }}$ either trace a narrow $\mathrm{H}_{\mathrm{I}}$ velocity distribution or $\mathrm{low}_{\mathrm{H}}$, both enhancing the observed emission of Ly $\alpha$ photons. However, the large $\mathrm{EW}(\mathrm{Ly} \alpha)$ in $\mathrm{J} 1248+4259$ likely results from a combination of several galaxy properties, such that disentangling its origin is not trivial.

Consequently, we exclude $\mathrm{J} 1248+4259$ from the sample and derive the equation that linearly relates $\mathrm{EW}(\mathrm{Ly} \alpha)$ and $1-C_{f}(\mathrm{HI})$ as $(4 \sigma$ significance level, p-value $<0.00002)$ :

$E W(\operatorname{Ly} \alpha)=165 \times\left(1-C_{f}(\mathrm{HI})\right)+17$.

We include this relation in Figure 10 (dotted line), and compare it with the one obtained by Steidel et al. (2018) (dashed line), where the authors investigated the connection between the neutral gas properties and the escape of $\operatorname{Ly} \alpha$ and LyC photons in composite spectra of $\mathrm{z} \sim 3$ galaxies observed with the Low Resolution Imaging Spectrometer (LRIS; Oke et al. 1995, Steidel et al. 2004) on the Keck I telescope. Both linear relations have similar slopes, but the relation from Steidel et al. (2018) slightly underestimates the $\mathrm{EW}(\mathrm{Ly} \alpha)$ from our sample. One reason for this is that Steidel et al. (2018) sample is drawn from composite spectra with $\mathrm{EW}(\operatorname{Ly} \alpha)<50 \AA$, while $\sim 60 \%$ of both our and the Jaskot et al. (2019); McKinney et al. (2019) samples have $\mathrm{EW}(\operatorname{Ly} \alpha)$ larger than $50 \mathrm{~A}$. Besides, the Ly $\alpha$ equivalent width is also known to relate to the aperture size of the instrument (Steidel et al. 2011; Hayes et al. 2014; Wisotzki et al. 2016), and therefore complicates direct comparison between samples observed with different instruments. Finally, the higher EW(Ly $\alpha)$ in our sample might be biased by different galaxy properties (i.e. stellar masses, velocity widths, etc) and because our sample was selected to be only galaxies with intense star formation and young stellar populations.

Although the physical interpretation of high $\operatorname{EW}(\operatorname{Ly} \alpha)$ is complex, the tight connection between $\operatorname{EW}(\mathrm{Ly} \alpha)$ and $1-C_{f}\left(\mathrm{H}_{\mathrm{I}}\right)$ is consistent with the findings from Sect. 4.1 and 4.2 and confirms the strong impact of low neutral gas coverage on the observed emission of Ly $\alpha$ photons.

\subsection{The escape of $L y \alpha$ and $L y C$ photons is larger in galaxies with lower $C_{f}(\mathrm{HI})$ and $E_{\mathrm{B}-\mathrm{V}}$}

Investigating the connection between the $\mathrm{H}_{\mathrm{I}}$ column density, $\mathrm{H} \mathrm{I}$ covering fraction, and the escape fraction of Ly $\alpha$ and LyC photons should allow us to understand the $\mathrm{H}$ i geometry that controls and regulates the leakage of these photons out of the ISM. Recently, we found saturated Lyman series with non-unity covering fraction in 9 LCEs included in this work, highlighting the existence of both low and high column density paths in galaxies that leak ionizing photons (Gazagnes et al. 2018). Additionally, we found that $6 \mathrm{LCEs}$ have $N_{\mathrm{H}_{\mathrm{I}}}$ measurements supporting the presence of optically thick regions $\left(>10^{18} \mathrm{~cm}^{-2}\right)$ in the ISM, which is incompatible with a density bounded scenario. Several studies similarly highlighted the close connection between $C_{f}\left(\mathrm{H}_{\mathrm{I}}\right)$ and/or $C_{f}(\mathrm{Si}$ II) and the Ly $\alpha$ escape fraction and equivalent width (Rivera-Thorsen et al.2015, Steidel et al.2018; McKinney et al. 2019, Jaskot et al. 2019).

In Fig. 11 we analyze the dependence between the covering fraction and the observed escape fractions of Ly $\alpha$ and LyC photons. We find a $4 \sigma$ correlation between $1-C_{f}\left(\mathrm{HI}_{\mathrm{I}}\right)$ and $f_{\mathrm{esc}}(\mathrm{Ly} \alpha)$ (p-value $<0.00002$ ) and a $3 \sigma$ significance level for the trend that connects $1-C_{f}(\mathrm{HI})$ to $f_{\mathrm{esc}}^{\mathrm{obs}}(\mathrm{LyC})$ (p-value of 0.0005$)$. Further, the left panel of Fig. 11 shows that most galaxies have a $f_{\text {esc }}(\mathrm{Ly} \alpha)$ lower than or equal to $1-C_{f}\left(\mathrm{H}_{\mathrm{I}}\right)$. For $f_{\mathrm{esc}}^{\mathrm{obs}}(\mathrm{LyC})$, the trend is slightly more scattered, but emphasizes that $1-C_{f}(\mathrm{HI})$ always overestimates the observed escape fraction of ionizing photons. This observational result is consistent with theoretical studies which suggest that $C_{f}\left(\mathrm{H}_{\mathrm{I}}\right)$ is always an upper limit to the escape of ionizing photons (Kakiichi \& Gronke 2019). Indeed, several reasons explain why the ionizing escape fraction should deviate from $1-C_{f}\left(\mathrm{HI}_{\mathrm{I}}\right)$ : the presence of $\mathrm{H}_{\mathrm{I}}$ residuals in the channels, the necessity to account for the dust attenuation (Chisholm et al. 2018), or because the covering fraction measured is always a lower limit to the true geometrical neutral gas covering (see discussion in Rivera-Thorsen et al. 2015, Vasei et al. 2016 Gazagnes et al. 2018, Mauerhofer et al, in prep). While these effects also impact the escape fraction of Ly $\alpha$ photons, Ly $\alpha$ photons have a higher chance to escape by being scattered and either shifted out of the velocity range of the optically thick neutral gas, or re-emitted in the direction of a low column density sightline. Hence, the kinematics of the H I gas can affect the escape of Ly $\alpha$ 

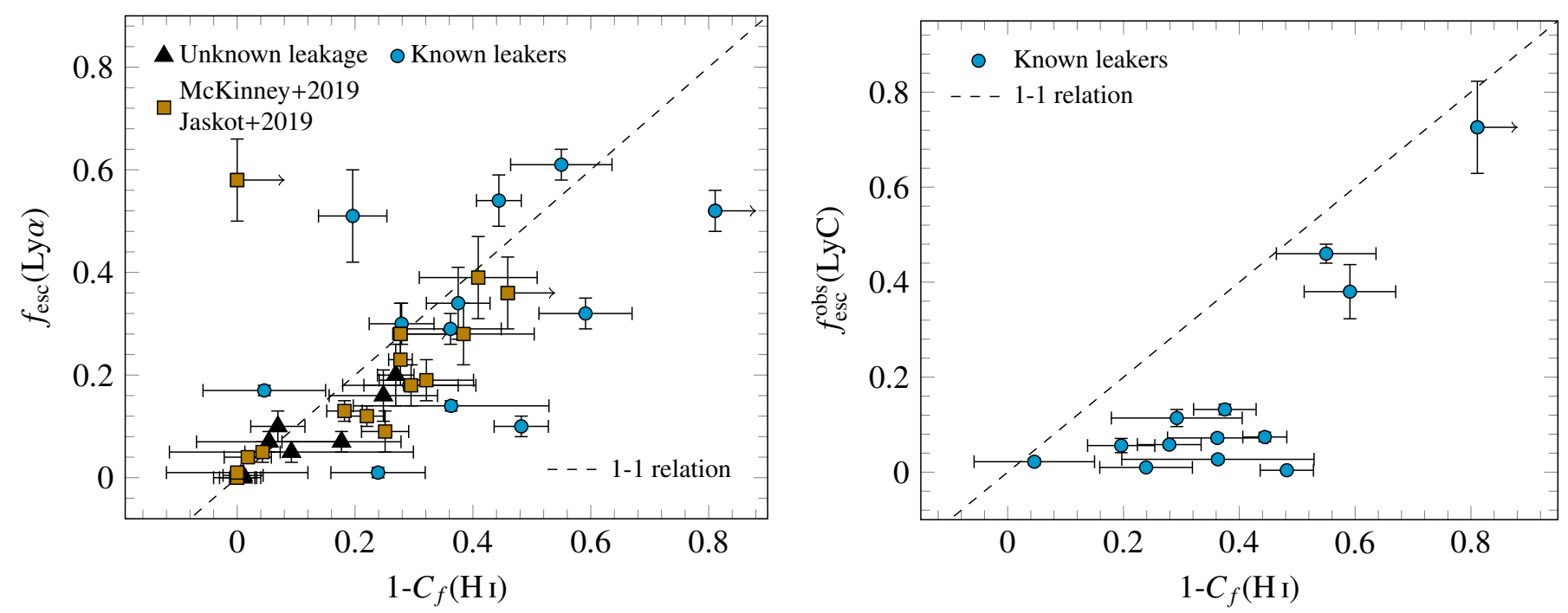

Fig. 11. Observed $f_{\mathrm{esc}}(\mathrm{Ly} \alpha)$ (left) and $f_{\mathrm{esc}}^{\mathrm{obs}}(\mathrm{LyC})$ (right) versus the 1- $C_{f}\left(\mathrm{H}_{\mathrm{I}}\right)$. There is a strong correlation between the escape of Ly $\alpha$ and LyC photons and the porosity of the $\mathrm{HI}_{\mathrm{I}}$ gas ( $4 \sigma$ and $3 \sigma$ level, respectively). The right panel shows that $1-C_{f}\left(\mathrm{H}_{\mathrm{I}}\right)$ is always an upper limit to the observed escape fraction of ionizing photons.
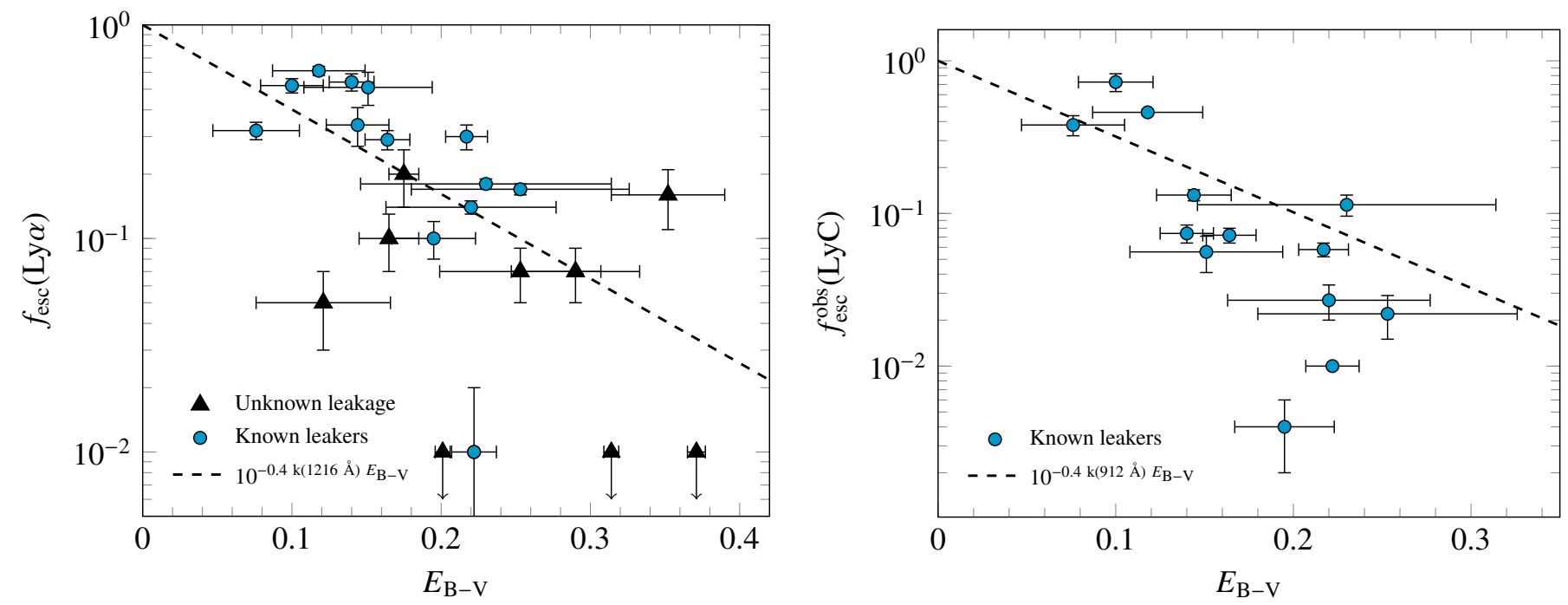

Fig. 12. Observed $f_{\text {esc }}(\mathrm{Ly} \alpha)$ (left) and $f_{\text {esc }}^{\text {obs }}($ LyC) (right) versus the dust extinction of the stellar continuum with logarithmic y-axes. We included the shape of the dust attenuation curve at the wavelength of interest (either Ly $\alpha$ or LyC) with a dashed line. The correlation between the escape fractions of $\mathrm{Ly} \alpha$ and LyC photons and the respective dust attenuation curve is significant at $3 \sigma$.

photons, while it does not impact the escape of LyC photons. This could explain why the neutral gas coverage is more correlated with the escape of Ly $\alpha$ photons. Further LyC observations are needed to confirm this trend. Nonetheless, these results confirm that the common origin of the leakage of $\mathrm{Ly} \alpha$ and $\mathrm{LyC}$ photons is the ISM porosity.

Additionally, we also investigated the impact of dust extinction on the escape fraction of $\operatorname{Ly} \alpha$ and LyC photons. Several studies have shown that dust readily destroys Ly $\alpha$ photons (Neufeld 1991; Hayes et al. 2011, Verhamme et al. 2015, 2017; Du et al. |2018, Kimm et al. 2019, Jaskot et al.|2019), while Chisholm et al. (2018) showed that the dust attenuation is a crucial ingredient for the escape of ionizing photons. On the other hand, recent $\operatorname{Ly} \alpha$-LyC simulations from Kimm et al. (2019) found that the presence of dust grains should not significantly af- fect the leakage of LyC photons. Hence, the true impact of dust on the ionizing radiation is still poorly understood.

In Fig. 12, we compare the escape fractions of $\operatorname{Ly} \alpha$ and LyC photons with the $E_{\mathrm{B}-\mathrm{V}}$ obtained from the stellar continuum UV fits. Both panels highlight that galaxies with large $f_{\text {esc }}(\mathrm{LyC})$ and $f_{\mathrm{esc}}(\mathrm{Ly} \alpha)$ have lower dust extinction. Interestingly, the three largest LyC leakers have the lowest $E_{\mathrm{B}-\mathrm{V}}$, suggesting that low amount of dust favors the escape of LyC photons. We superposed on the same figure the theoretical attenuation curve (dashed lines) given by $10^{-0.4 A(\lambda)}$, where $A(\lambda)$ is derived using the dust extinction law from Reddy et al. (2016a) at $\lambda=1216$ $\AA$ for Ly $\alpha$ photons and 912 A for LyC photons. We find a $3 \sigma$ significance level correlation between the dust attenuation and the escape fractions of LyC and Ly $\alpha$ photons (p-value of 0.0014 and 0.0011 , respectively). Hence, this indicates that the presence of interstellar dust grains in the ISM has a significant impact the 


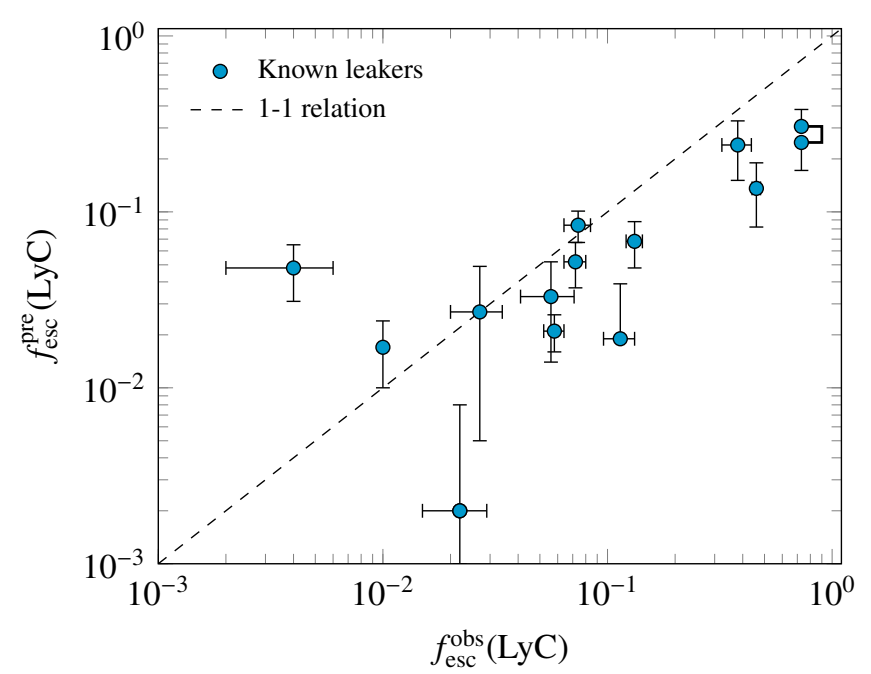

Fig. 13. Predicted LyC escape fraction $f_{\mathrm{esc}}^{\mathrm{pre}}(\mathrm{LyC})$, using the $\mathrm{H}_{\mathrm{I}}$ covering fraction and dust extinction, versus the observed $f_{\text {esc }}(\mathrm{LyC})$ plotted on a logarithmic scale. Dashed lines show the one-to-one relation. Two different predictions have been derived for J1243+4646 using $C_{f}(\mathrm{HI})=0.18$ (upper limit), and $C_{f}\left(\mathrm{H}_{\mathrm{I}}\right)=0$, and are shown on the upper right part of the Figure. The error bars on $f_{\mathrm{esc}}^{\mathrm{pre}}(\mathrm{LyC})$ are obtained by propagating the uncertainties from $C_{f}\left(\mathrm{H}_{\mathrm{I}}\right)$ and $E_{\mathrm{B}-\mathrm{V}}$. This Figure extends the work done in figure 1 of Chisholm et al. (2018) by including the 6 new LyC detections from Izotov et al. (2018a b).

escape of both types of emission. However, these results need to be taken with caution.

Figure 12 shows that a few galaxies have their Ly $\alpha$ or LyC escape fractions above the dust attenuation curve. In theory, in galaxies having no $\mathrm{H}_{\mathrm{I}}$ and a screen layer of dust, the fraction of $\mathrm{LyC}$ and $\mathrm{Ly} \alpha$ radiation escaping should be directly given by $10^{-0.4 A(\lambda)}$. Hence, no galaxies should have $f_{\mathrm{esc}}^{\mathrm{obs}}(\mathrm{LyC})$ or $f_{\mathrm{esc}}(\mathrm{Ly} \alpha)$ larger than the dust attenuation curve. Several reasons for this discrepancy were considered in Section 3.2. where we highlighted the complex task of measuring $E_{\mathrm{B}-\mathrm{V}}$, and showed that different dust extinction laws can lead in significant differences in the fitted attenuation at 1216 and $912 \AA$. Additionally, in this work, we use the Reddy et al. (2016a) dust attenuation curve, which is derived in the far-UV observations $(\lambda=950-1500 \AA)$. Nevertheless, the physical impact of dust extinction on the ionizing flux $(<912 \AA)$ is still unconstrained. Finally, the dust geometry model can also impact these results. A clumpy distribution of $\mathrm{H}$ I and dust could theoretically have a shielding effect such that Ly $\alpha$ photons have a lower probability to encounter dust (Neufeld 1991, Gronke et al. 2016), and enhance the observed emission of Ly $\alpha$ photons (Finkelstein et al. 2008). Scarlata et al. (2009) found that such clumpy distribution better reproduces the observed $\mathrm{Ly} \alpha / \mathrm{H} \alpha$ and $\mathrm{H} \alpha / \mathrm{H} \beta$ ratio in 31 low-z Ly $\alpha$ emitters. Hence, several factors can lead to different estimations of the dust impact, and robustly constraining the latter requires deeper observations. Furthermore, both the $f_{\mathrm{esc}}^{\mathrm{obs}}(\mathrm{LyC})$ and $f_{\mathrm{esc}}(\mathrm{Ly} \alpha)$ depend on accurately determining the intrinsic emission, which may be mis-estimated by our assumed models.

\subsection{Predicting the $L y C$ escape fraction from absorption line measurements}

The outcomes of Figs. 11 and 12 favor an ionization-bounded scenario, where dusty channels with low $N_{\mathrm{H}_{\mathrm{I}}}$ act as the dominant escape roads for $\mathrm{Ly} \alpha$ and $\mathrm{LyC}$ photons, and support the conclusions drawn in Gazagnes et al. (2018) and Chisholm et al. (2018). The latter work additionally proposed a novel approach to accurately recover the observed escape fractions based on the $C_{f}\left(\mathrm{H}_{\mathrm{I}}\right)$ and dust extinction. This indirect prediction method will be particularly useful at higher redshift where the flux at $<912 \AA$ can not be directly observed. Indeed, the upcoming James Space Webb Telescope (JWST) will have a sufficient resolution to observe low-ionization interstellar (LIS) absorption lines and measure $C_{f}\left(\mathrm{Si}\right.$ II), which can be then used to infer $C_{f}\left(\mathrm{H}_{\mathrm{I}}\right)$ (Gazagnes et al.2018). The analysis done in Chisholm et al. (2018) included 9 LCE 5 with $f_{\text {esc }}^{\mathrm{obs}}$ (LyC) between 1 and $13 \%$, and we test here its reliability using the six new leakers from Izotov et al. (2018ab), three of them with large LyC escape fractions $(\geq 38 \%)$.

This is shown in Fig. 13, where we plot the predicted escape fraction of LyC photons $\left(f_{\mathrm{esc}}^{\mathrm{pre}}(\mathrm{LyC})\right)$, computed as $(1-$ $\left.C_{f}(\mathrm{HI})\right) \times 10^{-0.4 A(\lambda=912 \AA)}$ (see Chisholm et al. 2018), versus the observed escape fraction of LyC photons $\left(f_{\mathrm{esc}}^{\mathrm{obs}}(\mathrm{LyC})\right)$, derived using the ratio of the observed flux at $\sim 900 \AA$ over the modelled flux obtained from SED fitting for the 13 confirmed LCEs in our sample. The recovered LyC escape fractions accurately match at $\pm 3 \sigma$ with $f_{\mathrm{esc}}^{\mathrm{obs}}(\mathrm{LyC})$ for 10 leakers, underestimates it for the 2 largest leakers, and overestimates it in Tol1247-232. For the latter, the bias is likely explained by a possible contamination from geocoronal emission as discussed in Chisholm et al. (2017a. 2018). We mentioned in Sect. 2 that several studies using different measurement methods have reported LyC escape fractions varying from 0.004 to 0.042 for Tol1247-232 (Leitherer et al. 2016. Chisholm et al. 2017a; Puschnig et al. 2017), which gives an interval consistent with the predicted $4.8 \pm 1.7 \%$. For the two largest leakers, several factors can explain the inconsistency between $f_{\mathrm{esc}}^{\mathrm{obs}}(\mathrm{LyC})$ and $f_{\mathrm{esc}}^{\mathrm{pre}}(\mathrm{LyC})$. The measurement of $f_{\mathrm{esc}}^{\mathrm{obs}}(\mathrm{LyC})$ can suffer from poor constraints on the estimation of intrinsic emission of LyC photons (Chisholm et al. 2019), and different $f_{\text {esc }}^{\text {obs }}(\mathrm{LyC})$ measurement approaches can lead to significant variations in some cases (for example $\mathrm{J} 1011+1947$ has $f_{\text {esc }}^{\text {obs }}($ LyC) $=$ $11.4 \pm 1.8 \%$ when using an approach based on SED fitting, and $f_{\mathrm{esc}}^{\mathrm{obs}}(\mathrm{LyC})=6.2 \pm 0.7 \%$ when using the $\mathrm{H} \beta$ flux density; Izotov et al. 2018b). Similarly, reliable measurements of the dust attenuation are required to accurately estimate $f_{\mathrm{esc}}^{\text {pre }}(\mathrm{LyC})$. We showed in Sect. 3.2 that a 0.1 difference in $E_{\mathrm{B}-\mathrm{V}}$ is a factor 2 different in attenuation, and hence in $f_{\mathrm{esc}}^{\mathrm{pre}}(\mathrm{LyC})$. Nevertheless, robustly constraining the stellar continuum and dust extinction is highly complicated in galaxies with low $\mathrm{S} / \mathrm{N}$ spectra (see Sect. 3.2.

Additionally, we showed in Gazagnes et al. (2018); Chisholm et al. (2018) that the dust geometry should not impact the derived $f_{\mathrm{esc}}^{\mathrm{prc}}(\mathrm{LyC})$ because only the combination of $C_{f}(\mathrm{HI})$ and $E_{\mathrm{B}-\mathrm{V}}$ changes to match the observed data. We refitted $\mathrm{J} 1154+2443$ and $\mathrm{J} 1256+4509\left(f_{\mathrm{esc}}^{\mathrm{obs}}(\mathrm{LyC})\right.$ of 0.46 and 0.38 , respectively) using a model where the dust lies only in the dense $\mathrm{H}_{\text {I }}$ clouds to check if this is still the case in large leakers. We found $f_{\mathrm{esc}}^{\mathrm{pre}}(\mathrm{LyC})=0.074 \pm 0.048$ and $0.242 \pm 0.095$ for J1154+2443 and J1256+4509 respectively, which are consistent with the predictions using the screen model $(0.136 \pm 0.054$ and $0.240 \pm 0.089$ ). Hence, assuming a different dust model does not improve the $f_{\text {esc }}($ LyC) estimation of galaxies with high $f_{\mathrm{esc}}^{\mathrm{obs}}(\mathrm{LyC})$.

Finally, indirectly predicting the ionizing escape in very strong leakers might be more complicated because their escape fraction could be a combination of the ionization and density bounded models, as suggested in Kakiichi \& Gronke (2019). The

\footnotetext{
$\overline{5}$ Two of them, Tol0440-381 and Mrk54 (Leitherer et al.2016), are not included in this work because they do not have Ly $\alpha$ observations
} 
authors showed that the leakage mechanisms in galaxies with a turbulent ISM dynamically evolves through time, such that the correlation between the leakage of LyC photons and the H I covering fraction becomes weaker as more and more channels, with lower $N_{\mathrm{H}_{\mathrm{I}}}^{\text {chanel }}$, form in the ISM. This is because these regions finally dominate the ISM such that the leakage is mostly regulated by the density-bounded scenario. While constraining the $N_{\mathrm{H}_{\text {I }}}$ requires large $\mathrm{S} / \mathrm{N}$ (Gazagnes et al.2018), the shape of the Ly $\alpha$ profile can provide insights of the dominant escape mechanisms. Kakiichi \& Gronke (2019) show that galaxies with a density bounded ISM have a lower red peak asymmetry $\left(A_{f}\right)$ (defined by Eq (33) in Kakiichi \& Gronke 2019) than galaxies with an ionization bounded ISM. In particular, they find that the three largest leakers in our sample have a red peak asymmetry consistent with galaxies with a density bounded ISM. Additionally, we showed in Sect. 4.2 that two of these leakers had the lowest $\mathrm{H}_{\mathrm{I}}$ velocity width of the maximal $\mathrm{H}_{\mathrm{I}}$ absorption, while we do not observe Hi absorption lines in the largest LCE. This supports the fact that galaxies with large $f_{\mathrm{esc}}^{\mathrm{obs}}(\mathrm{LyC})$ must also have lower H I column densities within the clouds that have the highest optical depth. In fact, Ramambason et al. (in preparation) find evidence from the optical emission lines pointing towards a density bounded ISM for these galaxies. This could explain why our approach based on $C_{f}\left(\mathrm{HI}_{\mathrm{I}}\right)$ underestimates the LyC escape fraction in these extreme leakers. In any case, Fig. 13 shows that the UV absorption lines can be used to determine a reliable lower limit of the escape of LyC photons in LCEs candidates.

Overall, our results suggest that the H I porosity and dust attenuation play a crucial role in the escape of $\mathrm{Ly} \alpha$ and $\mathrm{LyC}$ photons and $f_{\text {esc }}(\mathrm{LyC})$ predictions based on $C_{f}(\mathrm{HI})$ and $E_{\mathrm{B}-\mathrm{V}}$ can provide a lower limit on the $f_{\text {esc }}(\mathrm{LyC})$ along the line of sight, despite the potentially complex leakage mechanisms in LCEs with high LyC escape fraction. This indirect approach might be valuable for high-redshift observations, in order to compare the range of $f_{\text {esc }}(\mathrm{LyC})$ of galaxies during the Reionization era and hence whether star-forming galaxies reionized the Universe. We discuss this point further in Sect. 5.2.

\subsection{The flux at the Lyo profile minimum correlates with the LyC escape fraction}

Finally, we find that the presence of $\operatorname{Ly} \alpha$ emission at the profile trough is a valuable marker of peculiar neutral gas properties. Indeed, it suggests that a fraction of Ly $\alpha$ photons can escape unaffected where the optical depth of the H I gas should be maximal. In particular, a $\operatorname{Ly} \alpha$ profile with a single peak at the systemic velocity should emerge from any patchy ISM with fully cleared holes (no H I residuals), because all the Ly $\alpha$ photons should find these holes after a negligible amount of scattering events (Verhamme et al. 2015, Dijkstra et al. 2016). None of the galaxies in our sample with $C_{f}(\mathrm{HI})<1$ exhibit such Ly $\alpha$ spectral shape, including J1243+4646, which has no detected $\mathrm{H}$ I absorption (all Ly $\alpha$ profiles are shown in Appendix C). However, all LCEs have net flux at the Ly $\alpha$ trough, suggesting that some Ly $\alpha$ photons escaped without being scattered by the $\mathrm{H}_{\mathrm{I}}$ gas. This peculiarity has been observed in simulations in the presence of a very dense clumpy neutral gas distribution (Gronke et al. 2016, 2017), or when the Hi column density is low enough such that Ly $\alpha$ photons escape with fewer scattering events (Kakiichi \& Gronke 2019). In Sects. 4.1 and 4.2, we argued that $C_{f}(\mathrm{H} \mathrm{I})$ probes both the fraction of sightlines between the galaxy and the observer covered by low column density $\mathrm{H}_{\mathrm{I}}$ gas, such that $\mathrm{Ly} \alpha$ and LyC photons passing through these escape roads have a lower proba-

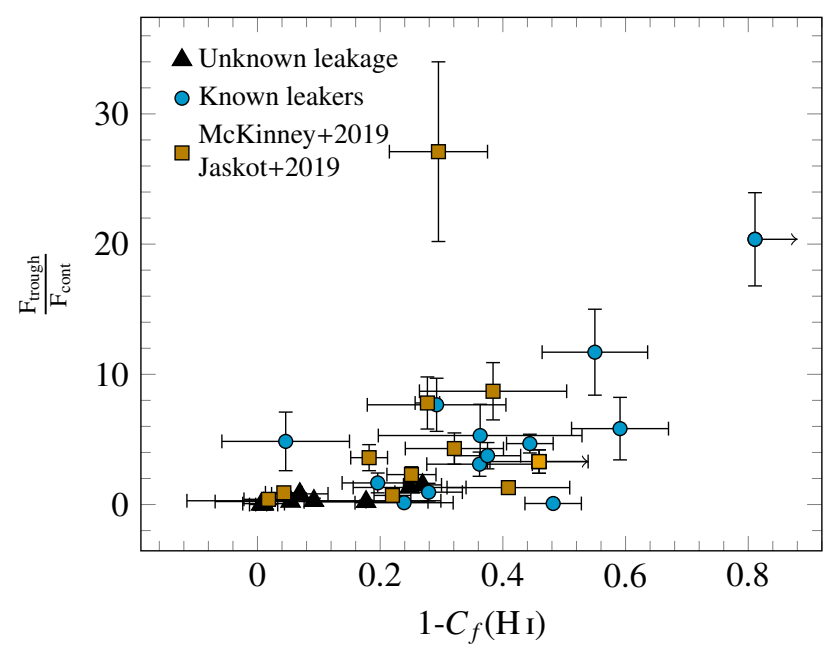

Fig. 14. The normalized $\operatorname{Ly} \alpha$ flux at the minimum of the $\operatorname{Ly} \alpha$ profile $\frac{\mathrm{F}_{\text {trough }}}{\mathrm{F}_{\text {cont }}}$ versus $1-C_{f}\left(\mathrm{H}_{\mathrm{I}}\right)$. The trend is scattered, but shows that galaxies with lower H covering fraction have higher residual flux at the Ly $\alpha$ profile minimum.

bility to interact with the neutral gas. Hence, a larger $\frac{F_{\text {trough }}}{F_{\text {cont }}}$ should correlate with the covering fraction of the low $N_{\mathrm{H}_{\mathrm{I}}}$ channels (1$C_{f}(\mathrm{HI})$ ), and with a larger escape fraction of ionizing photons.

Figure 14 explores the connection between $\frac{\mathrm{F}_{\text {trough }}}{\mathrm{F}_{\text {cont }}}$ and 1$C_{f}(\mathrm{HI})$ in our sample and in the GP sample studied by $\mathrm{McK}-$ inney et al. (2019) and Jaskot et al. (2019). The trend is slightly scattered, but the correlation is significant at the $3 \sigma$ (p-value of 0.0014). Hence, the presence of more flux at the Ly $\alpha$ trough is related to a lower H i covering fraction. In a highly porous ISM, higher amounts of $\operatorname{Ly} \alpha$ photons can escape at velocities where the $\mathrm{H}_{\mathrm{I}}$ optical depth is large because they find low column density sightlines (see Section 4.2). Note that, similar to Orlitová et al. (2018) and Jaskot et al. (2019), we report a strong correlation $(3.5 \sigma)$ between the Ly $\alpha$ peak velocity separation and the strength of the flux at the Ly $\alpha$ profile minimum (not shown). Hence, $v_{\mathrm{Ly} \alpha}^{\text {sep }}, \frac{\mathrm{F}_{\text {trough }}}{\mathrm{F}_{\text {cont }}}$ and $C_{f}\left(\mathrm{H}_{\mathrm{I}}\right)$ likely provide similar insights about the presence of channels with low $N_{\mathrm{H}}^{\text {channel }}$ in the ISM.

Additionally, we investigate in Fig. 15 the connection between $\frac{F_{\text {trough }}}{F_{\text {cont }}}$ and the escape of LyC photons. This Figure clearly highlights that all LCEs have net emission at the minimum profile, and larger $f_{\mathrm{esc}}^{\mathrm{obs}}(\mathrm{LyC})$ scales with larger $\frac{\mathrm{F}_{\text {trough }}}{\mathrm{F}_{\text {cont }}}$ (at the $3.5 \sigma$ level, p-value of 0.000022). Hence, similar to $v_{\mathrm{Ly} \alpha}^{\text {sep }}$ (Verhamme et al. 2017; Izotov et al. 2018b), $\frac{\mathrm{F}_{\text {trough }}}{\mathrm{F}_{\text {cont }}}$ is likely a reliable indicator of the presence of ISM properties that favor the leakage of ionizing photons. We derive the linear relation that relates $f_{\mathrm{esc}}(\mathrm{LyC})$ to $\frac{F_{\text {trough }}}{F_{\text {cont }}}$ in our sample (with $R \sim 15000$ observations from COS):

$f_{\text {esc }}(\mathrm{LyC})=(0.032 \pm 0.006) \times \frac{\mathrm{F}_{\text {trough }}}{\mathrm{F}_{\text {cont }}}-0.032 \pm 0.053$

In Gazagnes et al. (2018), we posit that J0926+4427, $\mathrm{J} 1429+0643$, and GP1054+5238 are potential LCE candidates, and estimated $f_{\mathrm{esc}}(\mathrm{LyC})$ of $\approx 1 \%$ using their $C_{f}(\mathrm{HI})$ and dust extinction measurements. Eq. (6) suggests that follow-up observations could measure LyC escape fractions of $<1 \%$ in GP1054+5238 and J1429+0643 ( $\frac{\mathrm{F}_{\text {trough }}}{\mathrm{F}_{\text {cont }}}$ of 0.21 and 0.82 respectively), and $1.6 \%$ in $\mathrm{J} 0926+4427\left(\frac{F_{\text {trough }}}{F_{\text {cont }}}=1.51\right)$. The largest mea- 


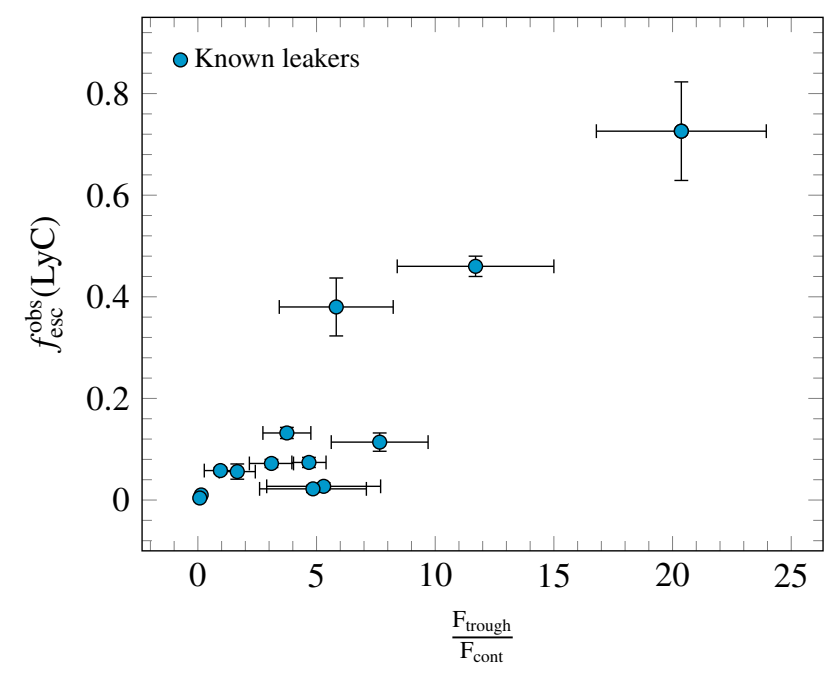

Fig. 15. Observed escape fraction of LyC photons versus $\frac{F_{\text {trough }}}{F_{\text {cont }}}$. Galaxies with higher residual flux at the Ly $\alpha$ profile minimum have a higher escape fraction of ionizing photons. Note that the Ly $\alpha$ trough depth is a resolution dependent measurement and requires a sufficient/high spectral resolution.

sured flux at minimum of Ly $\alpha$ profile in the GPs sample analyzed by McKinney et al. (2019) and Jaskot et al. (2019) is $\mathrm{J} 1608+3528$ with $\frac{\mathrm{F}_{\text {trough }}}{\mathrm{F}_{\text {cont }}}=27.1$, which corresponds to a very large $f_{\text {esc }}($ LyC) of $\approx 83 \%$ according to Eq. 6. Additionally, $\mathrm{J} 1608+3528$ also has a narrow peak velocity separation (214 +$30 \mathrm{~km} \mathrm{~s}^{-1}$, McKinney et al. 2019), which strongly supports the presence of LyC leakage.

Overall, Eq. (6) suggests that galaxies with $\frac{F_{\text {trough }}}{F_{\text {cont }}}>1$ have $f_{\text {esc }}($ LyC) $>1 \%$. Nevertheless, the reliability of Eq. (6) should be taken with caution because this linear relation is largely constrained by the large $f_{\text {esc }}(\mathrm{LyC}) \mathrm{LCEs}$, while the $\frac{\mathrm{F}_{\text {trough }}}{\mathrm{F}_{\text {cont }}}$ values of the low $f_{\text {esc }}($ LyC) LCEs are largely scattered. More observations, with sufficiently high spectral resolution, are needed to confirm its reliability.

\section{Discussion}

\subsection{The ISM porosity enables the escape of $L y \alpha$ and $L y C$ photons}

The observations of partially covered but saturated Lyman series absorption lines suggestan inhomogeneous ISM with both high and low column density H I gas. In this work, we studied the connection between the Ly $\alpha$ and the neutral gas properties and provided insights to understand how this bi-modal distribution of H I gas (e.g see Fig 6 of Kakiichi \& Gronke 2019) impacts the observed Ly $\alpha$ emission and LyC escape. Similar to recent studies (Rivera-Thorsen et al. 2015, Steidel et al. 2018, McKinney et al. 2019) that highlighted the connection between Ly $\alpha$ properties and the neutral gas covering fraction, we found that the observed emission and escape of Ly $\alpha$ photons of the 22 galaxies in our sample is closely connected to the fraction of sightlines between the galaxy and the observer covered by channels with low $N_{\mathrm{H}_{\mathrm{I}}}^{\text {chanel }}$ (Sects. 4.3 and 4.4. Additionally, Sect. 4.4 showed the close connection between the escape of ionizing photons and 1- $C_{f}\left(\mathrm{H}_{\mathrm{I}}\right)$, confirming that LCEs all have a non-unity $\mathrm{H}_{\mathrm{I}}$ covering fraction (Gazagnes et al. 2018). These results emphasize that there exists privileged escape roads for the Ly $\alpha$ and LyC radiation. More interestingly, we found in Sect. 4.1 that the Ly $\alpha$ peak separation strongly scales with the H I covering fraction. Because we expect $v_{\mathrm{Ly} \alpha}^{\text {sep }}$ to scale with $N_{\mathrm{H}}$ (Verhamme et al. 2015; Dijkstra et al. 2016), this relation may suggest that a low $C_{f}(\mathrm{HI})$ not only indicates the presence of low column density channels, but also lower $N_{\mathrm{HI}}^{\text {chanel }}$. This is similarly highlighted in Sect. 4.6 , where we showed that the $\operatorname{Ly} \alpha$ flux at the minimum of the $\operatorname{Ly} \alpha$ profile scales with $C_{f}(\mathrm{H}$ I). Hence, more Ly $\alpha$ photons escape unaffected by the $\mathrm{H}_{\mathrm{I}}$ gas when a larger fraction of low column density paths cut through the ISM.

In Gazagnes et al. (2018), we constrained $N_{\mathrm{H}_{\mathrm{I}}}$ in 6 low $f_{\text {esc }}^{\text {obs }}(\mathrm{LyC})$ leakers and found that the dense neutral regions have column densities large enough $\left(10^{18} \mathrm{~cm}^{-2}\right)$ to absorb all of the ionizing radiation. McKinney et al. (2019) reported similar insights from a sample of highly ionized Green Peas, and further emphasized that the $N_{\mathrm{H}_{\mathrm{I}}}$ derived from $N_{\mathrm{O}_{\mathrm{I}}}$ is always a lower limit to other indirect approaches where $N_{\mathrm{H} \text { I }}$ is derived from $N_{\mathrm{Si} \text { II }}$, or from the fit of the observed $\operatorname{Ly} \alpha$ absorption profile. Hence, these results show that, in these LCEs, ionizing photons escape through optically thin channels/holes in an ISM which is overall ionization bounded. Additionally, Sect. 4.5 highlighted that an indirect $f_{\text {esc }}(\mathrm{LyC})$ estimation method using $C_{f}\left(\mathrm{HI}_{\mathrm{I}}\right)$ and the dust extinction (Chisholm et al. 2018) accurately recovers the observed escape fractions in the low $f_{\text {esc }}($ LyC) LCEs.

Nevertheless, the latter approach underestimates $f_{\text {esc }}(\mathrm{LyC})$ in LCEs with the largest $f_{\text {esc }}^{\text {obs }}(\mathrm{LyC})$. This discrepancy could highlight that the escape mechanisms in these leakers can not be only explained by the presence of a low $C_{f}\left(\mathrm{H}_{\mathrm{I}}\right)$. In Sect. 4.2, we showed that the peak velocity separation is also tightly related to the velocity width of the maximal absorption of the saturated H I absorption lines. The connection between low $v_{\text {Ly } \alpha}^{\text {sep }}$ and low $N_{\mathrm{H}_{\text {I }}}$ (Verhamme et al. 2015, Dijkstra et al. 2016) could suggest that $v_{\mathrm{H}_{\mathrm{I}}}^{\text {widt }}$ indirectly probes the presence of lower $N_{\mathrm{H}_{\mathrm{I}}}^{\text {cloud }}$ within the densest neutral clouds in the ISM, such that a fraction of LyC photons escape through these regions. Interestingly, two of the three largest leakers (with $f_{\mathrm{esc}}^{\mathrm{obs}}(\mathrm{LyC})=38$ and $46 \%$ ) have the lowest $v_{\mathrm{HI}}^{\text {width }}$ in our sample, and $\mathrm{H}$ i is not detected in the largest LCE. Hence, for the largest leakers, a LyC prediction method using the covering fraction likely underestimates the total escape fraction of ionizing photons because all the lines of sight to the observer are density-bounded.

These outcomes are supported by the recent radiationhydrodynamics simulations of galaxies with a turbulent ISM by Kakiichi \& Gronke (2019). The authors showed that the early evolution of the turbulent gas kinematics generates channels with low $N_{\mathrm{H}}^{\text {chanel }}$, forming a ionization-bounded ISM where the leakage of LyC photons is directly driven by the fraction of paths covered by photo-ionized channels. Additionally, the evolution of the turbulent gas forms new channels and modifies the densities within these channels, increasing the fraction of low column density paths in the ISM. As a consequence, the escape mechanisms of LyC photons progressively evolve from a ionization bounded model $\left(f_{\text {esc }}(\mathrm{LyC})\right.$ directly proportional to $\left.C_{f}(\mathrm{HI})\right)$ towards a density bounded scenario (low $N_{\mathrm{H}_{\mathrm{I}}}$ in all the directions, weaker connection between $f_{\mathrm{esc}}(\mathrm{LyC})$ and $\left.C_{f}(\mathrm{HI})\right)$. The authors show that markers of this trade-off can be found on the Ly $\alpha$ profile, e.g using the red peak asymmetry $\left(A_{f}\right)$. Their simulations support that the largest leakers from Izotov et al. (2018a b) have $A_{f}$ consistent with a density bounded scenario, while leakers with $f_{\mathrm{esc}}^{\mathrm{obs}}(\mathrm{LyC})<13 \%$ have $A_{f}$ consistent with an ionization bounded model.

Overall these results provide new valuable insights to understand the dominant escape mechanisms of LyC photons. In the low LyC leakers, the ISM porosity enables the leakage of 


\section{a) low $f_{\text {esc }}^{\text {obs }}(\mathrm{LyC})$}

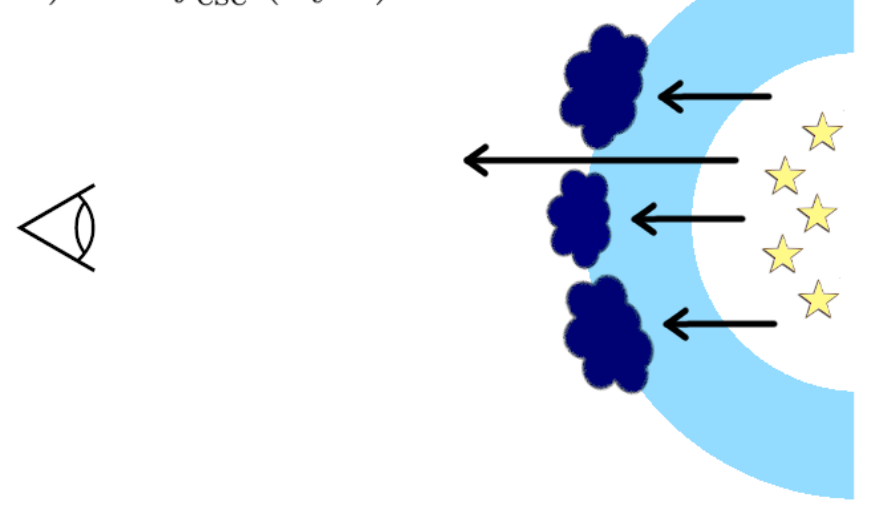

b) large $f_{\text {esc }}^{\text {obs }}(\mathrm{LyC})$

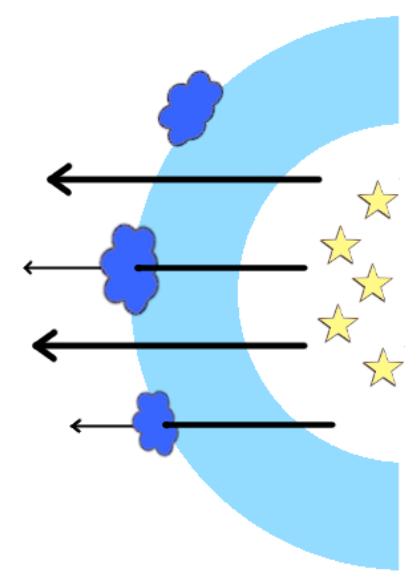

light sources

$N_{\mathrm{H}_{\mathrm{I}}}<10^{16} \mathrm{~cm}^{-2}$

$10^{16} \mathrm{~cm}^{-2}<N_{\mathrm{H}_{\mathrm{I}}}<10^{18} \mathrm{~cm}^{-2}$

$N_{\mathrm{HI}}>10^{18} \mathrm{~cm}^{-2}$

$\leftarrow$ LyC photons

Fig. 16. An illustration of the physical picture discussed in Sect. 5.1 Left: LyC photons escape through channels covered by $N_{\mathrm{H}_{\mathrm{I}}}^{\mathrm{chan} e l}<10^{16} \mathrm{~cm}^{-2}$, while $\mathrm{H}_{\mathrm{I}}$ clouds with $N_{\mathrm{H}_{\mathrm{I}}}^{\text {cloud }}$ larger than $10^{18} \mathrm{~cm}^{-2}$ efficiently absorb the ionizing flux that passes through them. The ISM is ionization bounded such that the escape of ionizing photons is directly proportional to the fraction of low column density paths (1- $C_{f}\left(\mathrm{H}_{\mathrm{I}}\right)$ ), and to the dust attenuation within or in front of these channels (note that we choose to exclude dust from this illustration because its spatial distribution is still unknown). This physical model likely describes the escape of ionizing photons from the low $f_{\mathrm{esc}}^{\text {obs }}(\mathrm{LyC})$ LCEs of our sample. Right: LyC photons escape because there is a higher fraction of low column density channels and because the densest $\mathrm{H}$ i clouds do not efficiently absorb LyC photons $\left(N_{\mathrm{H}}^{\text {cloud }}<10^{18}\right.$ $\left.\mathrm{cm}^{-2}\right)$. All the sightlines towards the observer are density-bounded such that the $f_{\mathrm{esc}}(\mathrm{LyC})$ cannot be inferred only using the measured $C_{f}\left(\mathrm{H}_{\mathrm{I}}\right)$. Our work suggests that this physical picture explains the escape of LyC photons in the large $f_{\mathrm{esc}}^{\text {obs }}(\mathrm{LyC})$ LCEs $(38,46$ and $73 \%)$ of our sample.

LyC and Ly $\alpha$ photons, such that the ionising photons predominantly escape through low column density paths in the ISM, while the densest neutral clouds have $N_{\mathrm{H} \text { I }}^{\text {cloud }}$ too large and efficiently absorb the LyC photons passing through them (Panel (a) of Fig. 16. However, the dynamical evolution of the ISM could shift the escape mechanisms towards a density bounded model, where all sightlines are covered by $N_{\mathrm{HI}_{\mathrm{I}}}<10^{17.9} \mathrm{~cm}^{-2}$ $\left(f_{\text {esc }}(\right.$ LyC $\left.) \approx 1 \%\right)$, enhancing the total escape fraction of LyC photons (Panel (b) of Fig. 16. This outcome emphasizes the need to constrain the $N_{\mathrm{HI}}$ in both the low and high density regions ( $N_{\mathrm{H}_{\mathrm{I}}}^{\text {chanel }}$ and $\left.N_{\mathrm{H}_{\mathrm{I}}}^{\text {cloud }}\right)$ to obtain accurate LyC predictions. Figure 16 illustrates the two different ISM configurations for small and large $f_{\text {esc }}^{\text {obs }}(\mathrm{LyC})$ LCEs discussed in this Section. Further low-redshift LyC observations should be able to assess this model.

\subsection{Indirectly constraining $f_{\mathrm{esc}}(\mathrm{LyC})$ at high-z}

The upcoming era of telescopes such as JWST and Extremely Large Telescopes (ELTs; GMT, TMT, ELT) will probe galaxies within the Epoch of Reionization and provide valuable clues to understand how the Universe was re-ionized. However, preparing for future observations requires finding pre-selection markers to efficiently survey large samples of LyC candidates and indirectly constrain their $f_{\text {esc }}$ (LyC). To do this, low-redshift observations of the Lyman break in highly star-forming galaxies provides a unique laboratory of LCEs (Borthakur et al. 2014; Izotov et al. 2016b a, 2018a b; Wang et al. 2019) to explore the connection between the Ly $\alpha$, LyC and neutral gas properties. In this work, we highlighted that the neutral gas porosity is the plausible physical origin of the leakage of LyC and Ly $\alpha$ photons, but that density-bounded mechanisms could dominate in the largest leakers. Because the ISM porosity affects the shape of the Ly $\alpha$ emission, Ly $\alpha$ properties can provide valuable insights about the leakage of LyC photons. The scaling relations between $v_{\mathrm{Ly} \alpha}^{\mathrm{sep}}, N_{\mathrm{H}}$ and $C_{f}\left(\mathrm{H}_{\mathrm{I}}\right)$ (Sect. 4.1) confirms that low peak velocity separation is likely a reliable probe of LyC leakage (Verhamme et al. 2017, Izotov et al. 2018b, 2020). However, deriving an accurate estimate of $f_{\text {esc }}(\mathrm{LyC})$ from it might be complicated because the Ly $\alpha$ spectral shape is both sensitive to the covering fraction of low column density channels, and to the width of the maximal absorption of the saturated Lyman series (Sect. 4.2). The positive correlation found between the red peak velocity and the $\mathrm{H}_{\mathrm{I}}$ covering fraction might also support the use of $v_{\mathrm{Ly} \alpha}^{\mathrm{red}}$ to probe the escape of LyC photons, but further theoretical simulations are required to understand its origin.

Additionally, we showed that $\operatorname{EW}(\operatorname{Ly} \alpha)$ or $f_{\mathrm{esc}}(\operatorname{Ly} \alpha)$ scale with $C_{f}\left(\mathrm{HI}_{\mathrm{I}}\right)$ (Sect. 4.3 and 4.4) and therefore could also indicate LyC leakage. Nevertheless, the $\operatorname{EW}(\operatorname{Ly} \alpha)$ is also tightly connected to the velocity width of the maximal absorption of the Lyman series (Figs. 7 and 10, and is degenerate with different galaxy properties such as the star formation history or metallicity. Hence, galaxies with large $\mathrm{EW}(\operatorname{Ly} \alpha)$ may have moderate $f_{\text {esc }}(\operatorname{Ly} \alpha)$ and $f_{\text {esc }}($ LyC) (Jaskot et al. 2019). On the other hand, Izotov et al. (2020) derived an empirical relation between $f_{\text {esc }}(\mathrm{LyC})$ and $f_{\text {esc }}(\operatorname{Ly} \alpha)$. Because both escape through channels with low $N_{\mathrm{H}_{\mathrm{I}}}$, it gives confidence that such connection could be useful to indirectly derive $f_{\text {esc }}(\mathrm{LyC})$ despite the complex task of accurately measuring $f_{\mathrm{esc}}(\mathrm{Ly} \alpha)$ due to scattering mechanisms and biases introduced from aperture corrections.

Finally, we showed in Sect. 4.6 that the strength of the flux at the minimum of the Ly $\alpha$ profile is, similarly to the peak velocity separation, a robust tracer of the leakage of ionizing photons. Hence, our work supports the use of the Ly $\alpha$ properties to robustly pre-select LCEs candidate at low-redshift, and further constrain their $f_{\text {esc }}(\mathrm{LyC})$ using direct observations of the Lyman Break when possible.

However, probing the LyC escape with the Ly $\alpha$ properties at high redshift is more challenging. Wavelengths bluer than Ly $\alpha$ are highly contaminated by $\operatorname{Ly} \alpha$ forest, such that $\operatorname{Ly} \alpha$ diagnos- 
tics are less reliable (Schenker et al. 2014). Quantities such as $v_{\mathrm{Ly} \alpha}^{\text {sep }}$ and $\frac{\mathrm{F}_{\text {trough }}}{\mathrm{F}_{\text {cont }}}$ may not be accurately measured and different approaches must be found. At these high redshifts, the study of the LIS metal lines likely provide better constrains on the presence of favorable ISM properties for the LyC escape. LIS lines are often used as proxies to extract the neutral gas properties (Shapley et al. 2003; Jones et al. 2013; Alexandroff et al. 2015; Trainor et al. 2015; Reddy et al. 2016b; McKinney et al. 2019; Jaskot et al. 2019). Indeed, they are located at redder wavelengths and hence can be more easily observed at higher redshift. On the other hand, LIS metal lines also suffer some disadvantages, as they are potentially affected by scattering, fluorescent emission in-filling, and they often require high resolution observations to be fully resolved. Despite these caveats, they are currently one of the best LyC probes we have at high redshift. McKinney et al. (2019) and Jaskot et al. (2019) recently showed that LIS lines provide similar insights as Ly $\alpha$ properties.

In Sect. 4.2, we highlighted the tight relation between the Ly $\alpha$ peak velocity separation and the velocity width of the saturated Lyman series. Chisholm et al. (2016) showed that different ionic transitions have similar velocity widths, thus, the Si II velocity width could provide an indirect estimation of $v_{\text {Ly } \alpha}^{\text {sep }}$ and indirectly probe the escape of ionizing photons. Similarly, in Gazagnes et al. (2018) and Chisholm et al. (2018), we showed that $C_{f}(\mathrm{Si}$ II $)$ can be used to empirically recover $C_{f}\left(\mathrm{H}_{\mathrm{I}}\right)$ and then combined to the dust attenuation at $912 \AA$ to indirectly infer $f_{\text {esc }}(\mathrm{LyC})$. Section 4.5 showed that the latter approach was less accurate for galaxies with large observed escape fractions, likely because of the presence of more complex leakage mechanisms, such that the total $f_{\text {esc }}($ LyC) along the line of sight cannot be inferred only using the neutral gas coverage (Kakiichi \& Gronke 2019). Additionally, the strong trends found between the H I covering fraction and the escape of Ly $\alpha$ and LyC photons suggest that $C_{f}(\mathrm{HI})$ is a good proxy of the true geometric covering fraction of the optically thick neutral gas clouds along the line of sight for the LCEs in our sample. As mentioned above, this might not be always the case because kinematic effects might bias the $C_{f}$ measurements towards lower values. Despite these caveats, it remains a reliable approach to derive a lower limit to the true LyC escape fraction in compact star-forming galaxies where these effects might have less of an impact. Hence, it should provide valuable constraints on the expected impact of these galaxies during the epoch of reionization.

Upcoming observations will allow us to confirm the robustness of the different pre-selection and indirect estimation approaches discussed in this section.

\section{Summary}

We have examined the neutral gas properties of a sample of 22 star-forming galaxies that have Ly $\alpha$ and Lyman series observations. We fitted the stellar continua, dust attenuations, H I covering fractions, $\mathrm{H}_{\mathrm{I}}$ velocity shifts and measured the Ly $\alpha$ properties, the H I velocity widths and depths of the Lyman series using a Monte-Carlo approach. We investigated the relations between the neutral gas properties in galaxies with the escape and emission of $\operatorname{Ly} \alpha$ photons and the observed LyC escape fractions. We summarize our results as follow:

- Ly $\alpha$ photons are less scattered in galaxies with with a larger fraction of low density sightlines towards the observer (Sect. 4.1). Additionally, the scaling relation found between the Ly $\alpha$ peak velocities and the $\mathrm{H}$ I covering fractions $\left(C_{f}\left(\mathrm{H}_{\mathrm{I}}\right)\right)$ suggests that galaxies with lower neutral gas coverage also have lower Hi column densities in the lowdensity channels (Fig. 6). Thus, low-z LCEs have small Ly $\alpha$ peak separations because photons find low column density escape roads out of the galaxy.

- The velocity width of the Hi gas, measured at the maximal depth of the $\mathrm{H}_{\mathrm{I}}$ absorption lines, has a significant impact on the Ly $\alpha$ emission, escape and velocity shift (Sect. 4.2). Galaxies with narrower $\mathrm{H}_{\mathrm{I}}$ absorption lines have higher $\mathrm{EW}(\operatorname{Ly} \alpha)$ and larger $f_{\mathrm{esc}}(\operatorname{Ly} \alpha)\left(\mathrm{Fig} 10\right.$. Additionally, $v_{\mathrm{Ly} \alpha}^{\text {sep }}$ linearly scales with $v_{\mathrm{H}_{\mathrm{I}}}^{\text {width }}$, and is lower in galaxies with lower $\mathrm{H}$ I covering fraction (Fig. 7). This highlights that the shape of the Ly $\alpha$ profile relates both to the presence of the low column density channels (low $C_{f}\left(\mathrm{HI}_{\mathrm{I}}\right)$ ) and to the properties of the denser neutral clouds imprinted from the saturated $\mathrm{H}_{\mathrm{I}}$ absorption lines $\left(v_{\mathrm{H}_{\mathrm{I}}}^{\text {width }}\right)$. The presence of low $v_{\mathrm{HI}_{\mathrm{I}}}^{\text {width }}$ in the galaxies with the highest $f_{\mathrm{esc}}^{\mathrm{obs}}(\mathrm{LyC})$ may suggest that they have overall less H i gas.

- Galaxies with lower covering fractions and/or dust attenuations have larger Ly $\alpha$ and LyC escape fractions (Figs. 11 and 12 . The presence of a porous ISM $\left(C_{f}(\mathrm{HI})<1\right)$ is a necessary condition to observe a significant leakage of ionizing radiation (Fig. 13). At high-redshift, the $C_{f}(\mathrm{Si}$ II) can be used to estimate $C_{f}\left(\mathrm{H}_{\mathrm{I}}\right)$ and provide valuable insights about the presence of LyC leakage. Additionally, using 6 recently observed LCEs, we found that the indirect approach to measure the line of sight $f_{\text {esc }}(\mathrm{LyC})$ proposed in Chisholm et al. (2018) accurately recovers their observed $f_{\text {esc }}($ LyC) within $\pm 3 \sigma$ for 9 leakers with $f_{\mathrm{esc}}^{\mathrm{obs}}(\mathrm{LyC})<13 \%$, and yields a lower limit on the total observed escape fractions in the LCEs with the largest LyC escape fractions. We suggest that recovering $f_{\text {esc }}^{\text {obs }}(\mathrm{LyC})$ in these extreme leakers is more complex because their LyC leakage combines ionization and density bounded mechanisms (Fig. 16). Hence, an indirect prediction methods based only on $C_{f}\left(\mathrm{H}_{\mathrm{I}}\right)$ sets a lower limit to the true LyC escape fractions.

- Galaxies with lower $C_{f}\left(\mathrm{H}_{\mathrm{I}}\right)$ have a larger flux at the minimum of the Ly $\alpha$ profile (Fig. 14), and the Ly $\alpha$ profile minima strongly scales with the escape fraction of LyC photons (Fig. 15). Therefore, the presence of low-density paths in the ISM is presumably the common origin of low $v_{\text {Ly } \alpha}^{\text {sep }}$ and high $\frac{F_{\text {trough }}}{F_{\text {cont }}}$ in the LCEs in our sample.

Our work emphasizes that the ISM porosity plays a major role in understanding the origin of the Ly $\alpha$ properties and the escape of LyC photons in LCEs, but additional escape mechanisms might be needed to explain the LyC escape fractions observed in the leakers with the largest LyC escape fractions (Fig. 16. Additionally, it shows that the Ly $\alpha$ spectral shape, in particular through low $v_{\mathrm{Ly} \alpha}^{\text {sep }}$ and high $\frac{\mathrm{F}_{\text {trough }}}{\mathrm{F}_{\text {cont }}}$, probes ISM properties that favor the leakage of Ly $\alpha$ and LyC photons. At high redshift, the use of LIS lines is likely more appropriate to detect and constrain the ionizing escape fraction in Reionization era galaxies. Indeed, Si II can be used to infer $C_{f}(\mathrm{HI})$ at high redshift (Gazagnes et al. 2018), and hence combined with $E_{\mathrm{B}-\mathrm{V}}$ to derive a lower limit of $f_{\text {esc }}($ LyC) using the procedure from Chisholm et al. (2018). Recent additional approaches could further constrain the LyC escape fractions in currently confirmed LCEs (e.g using Mg II; Chisholm et al, in prep, Henry et al. 2018). Therefore LIS diagnostics seems promising for future prospects at higher redshift, to detect LyC leaking candidates at high redshift $(z>6)$ with the upcoming era of the telescopes probing the Epoch of Reionization. 
Acknowledgements. The authors would like to thank the referee for the comments that helped improve the quality of this paper. The authors also thank Jed McKinney and Emil Rivera-Thorsen for the useful comments provided on a draft version of this paper. The position of SG was funded from a grant by the Center for Data Science and Systems Complexity (DSSC), University of Groningen. YI acknowledges support from the National Academy of Sciences of Ukraine by its priority project No. 0120U100935 "Fundamental properties of the matter in the relativistic collisions of nuclei and in the early Universe". Support for this work was provided by NASA through the NASA Hubble Fellowship gran \#51432 awarded by the Space Telescope Science Institute, which is operated by the Association of Universities for Research in Astronomy, Inc., for NASA, under contract NAS5-26555.

\section{References}

Alexandroff, R. M., Heckman, T. M., Borthakur, S., Overzier, R., \& Leitherer, C. 2015, ApJ, 810, 104

Bergvall, N., Zackrisson, E., Andersson, B.-G., et al. 2006, A\&A, 448, 513

Bian, F., Fan, X., McGreer, I., Cai, Z., \& Jiang, L. 2017, ApJ, 837, L12

Borthakur, S., Heckman, T. M., Leitherer, C., \& Overzier, R. A. 2014, Science, 346,216

Byler, N., Dalcanton, J. J., Conroy, C., et al. 2018, ApJ, 863, 14

Calzetti, D., Armus, L., Bohlin, R. C., et al. 2000, ApJ, 533, 682

Cardelli, J. A., Clayton, G. C., \& Mathis, J. S. 1989, in IAU Symposium, Vol 135, Interstellar Dust, ed. L. J. Allamandola \& A. G. G. M. Tielens, 5-10

Chisholm, J., Gazagnes, S., Schaerer, D., et al. 2018, A\&A, 616, A30

Chisholm, J., Orlitová, I., Schaerer, D., et al. 2017a, A\&A, 605, A67

Chisholm, J., Rigby, J. R., Bayliss, M., et al. 2019, ApJ, 882, 182

Chisholm, J., Tremonti, C. A., Leitherer, C., \& Chen, Y. 2017b, MNRAS, 469, 4831

Chisholm, J., Tremonti, C. A., Leitherer, C., Chen, Y., \& Wofford, A. 2016, MNRAS, 457, 3133

de Barros, S., Vanzella, E., Amorín, R., et al. 2016, A\&A, 585, A51

Dijkstra, M., Gronke, M., \& Venkatesan, A. 2016, ApJ, 828, 71

Dressler, A., Henry, A., Martin, C. L., et al. 2015, ApJ, 806, 19

Du, X., Shapley, A. E., Reddy, N. A., et al. 2018, ApJ, 860, 75

Ferland, G. J., Chatzikos, M., Guzmán, F., et al. 2017, Rev. Mexicana Astron. Astrofis., 53, 385

Ferland, G. J., Porter, R. L., van Hoof, P. A. M., et al. 2013, Rev. Mexicana Astron. Astrofis, 49, 137

Finkelstein, S. L., D’ Aloisio, A., Paardekooper, J.-P., et al. 2019, ApJ, 879, 36

Finkelstein, S. L., Rhoads, J. E., Malhotra, S., Grogin, N., \& Wang, J. 2008, ApJ, 678,655

Fletcher, T. J., Tang, M., Robertson, B. E., et al. 2019, ApJ, 878, 87

Fontanot, F., Cristiani, S., Pfrommer, C., Cupani, G., \& Vanzella, E. 2014, MNRAS, 438, 2097

Fontanot, F., Cristiani, S., \& Vanzella, E. 2012, MNRAS, 425, 1413

Gazagnes, S., Chisholm, J., Schaerer, D., et al. 2018, A\&A, 616, A29

Green, J. C., Froning, C. S., Osterman, S., et al. 2012, ApJ, 744, 60

Gronke, M., Dijkstra, M., McCourt, M., \& Oh, S. P. 2016, ApJ, 833, L26

Gronke, M., Dijkstra, M., McCourt, M., \& Oh, S. P. 2017, A\&A, 607, A71

Hayes, M., Östlin, G., Duval, F., et al. 2014, ApJ, 782, 6

Hayes, M., Schaerer, D., Östlin, G., et al. 2011, ApJ, 730, 8

Heckman, T. M., Alexandroff, R. M., Borthakur, S., Overzier, R., \& Leitherer, C. 2015, ApJ, 809, 147

Heckman, T. M., Borthakur, S., Overzier, R., et al. 2011, ApJ, 730, 5

Heckman, T. M., Lehnert, M. D., Strickland, D. K., \& Armus, L. 2000, ApJS, 129,493

Henry, A., Berg, D. A., Scarlata, C., Verhamme, A., \& Erb, D. 2018, ApJ, 855, 96

Henry, A., Scarlata, C., Martin, C. L., \& Erb, D. 2015, ApJ, 809, 19

Izotov, Y. I., Guseva, N. G., \& Thuan, T. X. 2011, ApJ, 728, 161

Izotov, Y. I., Orlitová, I., Schaerer, D., et al. 2016a, Nature, 529, 178

Izotov, Y. I., Schaerer, D., Thuan, T. X., et al. 2016b, MNRAS, 461, 3683

Izotov, Y. I., Schaerer, D., Worseck, G., et al. 2018a, MNRAS, 474, 4514

Izotov, Y. I., Schaerer, D., Worseck, G., et al. 2020, MNRAS, 491, 468

Izotov, Y. I., Worseck, G., Schaerer, D., et al. 2018b, MNRAS, 478, 4851

Jaskot, A. E., Dowd, T., Oey, M. S., Scarlata, C., \& McKinney, J. 2019, ApJ, 885,96

Jones, T. A., Ellis, R. S., Schenker, M. A., \& Stark, D. P. 2013, ApJ, 779, 52

Kakiichi, K. \& Gronke, M. 2019, arXiv e-prints, arXiv:1905.02480

Kimm, T., Blaizot, J., Garel, T., et al. 2019, MNRAS, 486, 2215

Koester, B. P., Gladders, M. D., Hennawi, J. F., et al. 2010, ApJ, 723, L73

Leitet, E., Bergvall, N., Hayes, M., Linné, S., \& Zackrisson, E. 2013, A\&A, 553, A106

Leitherer, C., Hernandez, S., Lee, J. C., \& Oey, M. S. 2016, ApJ, 823, 64

Leitherer, C., Ortiz Otálvaro, P. A., Bresolin, F., et al. 2010, ApJS, 189, 309

Leitherer, C., Schaerer, D., Goldader, J. D., et al. 1999, ApJS, 123, 3
Madau, P. \& Haardt, F. 2015, ApJ, 813, L8

Markwardt, C. B. 2009, in Astronomical Society of the Pacific Conference Series, Vol. 411, Astronomical Data Analysis Software and Systems XVIII, ed. D. A. Bohlender, D. Durand, \& P. Dowler, 251

Marshall, J. L., Burles, S., Thompson, I. B., et al. 2008, in Proc. SPIE, Vol. 7014, Ground-based and Airborne Instrumentation for Astronomy II, 701454

McKinney, J. H., Jaskot, A. E., Oey, M. S., et al. 2019, ApJ, 874, 52

Meynet, G., Maeder, A., Schaller, G., Schaerer, D., \& Charbonnel, C. 1994, A\&AS, 103, 97

Mitra, S., Choudhury, T. R., \& Ferrara, A. 2018, MNRAS, 473, 1416

Neufeld, D. A. 1991, ApJ, 370, L85

Oke, J. B., Cohen, J. G., Carr, M., et al. 1995, PASP, 107, 375

Orlitová, I., Verhamme, A., Henry, A., et al. 2018, A\&A, 616, A60

Osterbrock, D. E. 1989, Astrophysics of gaseous nebulae and active galactic nuclei

Ouchi, M., Mobasher, B., Shimasaku, K., et al. 2009, ApJ, 706, 1136

Pettini, M. \& Pagel, B. E. J. 2004, MNRAS, 348, L59

Pettini, M., Rix, S. A., Steidel, C. C., et al. 2002, ApJ, 569, 742

Prochaska, J. X., Kasen, D., \& Rubin, K. 2011, ApJ, 734, 24

Puschnig, J., Hayes, M., Östlin, G., et al. 2017, MNRAS, 469, 3252

Reddy, N. A., Kriek, M., Shapley, A. E., et al. 2015, ApJ, 806, 259

Reddy, N. A., Steidel, C. C., Pettini, M., \& Bogosavljević, M. 2016a, ApJ, 828, 107

Reddy, N. A., Steidel, C. C., Pettini, M., Bogosavljević, M., \& Shapley, A. E. 2016b, ApJ, 828, 108

Rigby, J. R., Bayliss, M. B., Sharon, K., et al. 2018, AJ, 155, 104

Rivera-Thorsen, T. E., Dahle, H., Chisholm, J., et al. 2019, Science, 366, 738

Rivera-Thorsen, T. E., Hayes, M., Östlin, G., et al. 2015, ApJ, 805, 14

Robertson, B. E., Ellis, R. S., Furlanetto, S. R., \& Dunlop, J. S. 2015, ApJ, 802, L19

Robertson, B. E., Furlanetto, S. R., Schneider, E., et al. 2013, ApJ, 768, 71

Runnholm, A., Hayes, M., Melinder, J., et al. 2020, ApJ, 892, 48

Scarlata, C., Colbert, J., Teplitz, H. I., et al. 2009, ApJ, 704, L98

Scarlata, C. \& Panagia, N. 2015, ApJ, 801, 43

Schaerer, D. 2003, A\&A, 397, 527

Schenker, M. A., Ellis, R. S., Konidaris, N. P., \& Stark, D. P. 2014, ApJ, 795, 20

Shapley, A. E., Steidel, C. C., Pettini, M., \& Adelberger, K. L. 2003, ApJ, 588, 65

Shapley, A. E., Steidel, C. C., Strom, A. L., et al. 2016, ApJ, 826, L24

Stark, D. P., Swinbank, A. M., Ellis, R. S., et al. 2008, Nature, 455, 775

Stasińska, G. \& Leitherer, C. 1996, ApJS, 107, 661

Steidel, C. C., Bogosavljević, M., Shapley, A. E., et al. 2011, ApJ, 736, 160

Steidel, C. C., Bogosavljević, M., Shapley, A. E., et al. 2018, ApJ, 869, 123

Steidel, C. C., Shapley, A. E., Pettini, M., et al. 2004, ApJ, 604, 534

Steidel, C. C., Strom, A. L., Pettini, M., et al. 2016, ApJ, 826, 159

Trainor, R. F., Steidel, C. C., Strom, A. L., \& Rudie, G. C. 2015, ApJ, 809, 89

Trainor, R. F., Strom, A. L., Steidel, C. C., et al. 2019, ApJ, 887, 85

Vanzella, E., de Barros, S., Castellano, M., et al. 2015, A\&A, 576, A116

Vasei, K., Siana, B., Shapley, A. E., et al. 2016, ApJ, 831, 38

Verhamme, A., Orlitová, I., Schaerer, D., \& Hayes, M. 2015, A\&A, 578, A7

Verhamme, A., Orlitová, I., Schaerer, D., et al. 2017, A\&A, 597, A13

Wakker, B. P., Hernandez, A. K., French, D. M., et al. 2015, ApJ, 814, 40

Wang, B., Heckman, T. M., Leitherer, C., et al. 2019, ApJ, 885, 57

Weiner, B. J., Coil, A. L., Prochaska, J. X., et al. 2009, ApJ, 692, 187

Wisotzki, L., Bacon, R., Blaizot, J., et al. 2016, A\&A, 587, A98

Worseck, G., Prochaska, J. X., Hennawi, J. F., \& McQuinn, M. 2016, ApJ, 825, 144

Wuyts, E., Rigby, J. R., Gladders, M. D., et al. 2012, ApJ, 745, 86

Zackrisson, E., Inoue, A. K., \& Jensen, H. 2013, ApJ, 777, 39

\section{Appendix A: Fits}

In Figs A.1 A.5 we present the fits for the galaxies J1154+2443, $\mathrm{J} 0901+2119, \mathrm{~J} 1011+1947, \mathrm{~J} 1243+4646$ and $\mathrm{J} 1248+4259$ from Izotov et al. (2018a b) that have been included in this sample. The fit of J1256+4509 is shown in Fig. 2, while the fits for the 16 other galaxies can be found in Gazagnes et al. (2018). Each figure shows the full wavelength coverage; the observed flux is in black, the green line shows the error on the observed flux and the resulting fit is in blue. The gray shaded areas show the main regions that were masked during the fit because of geo-coronal emission, low S/N, ISM or Milky Way absorption lines not included in the fit, or Ly $\alpha$ emission. In the top panels, we zoom in on individual Lyman series lines and denote the fitted Lyman series lines. Note that for display purposes, the masks on the ISM or Milky Way absorption lines are only shown when they appear in the top panels. 


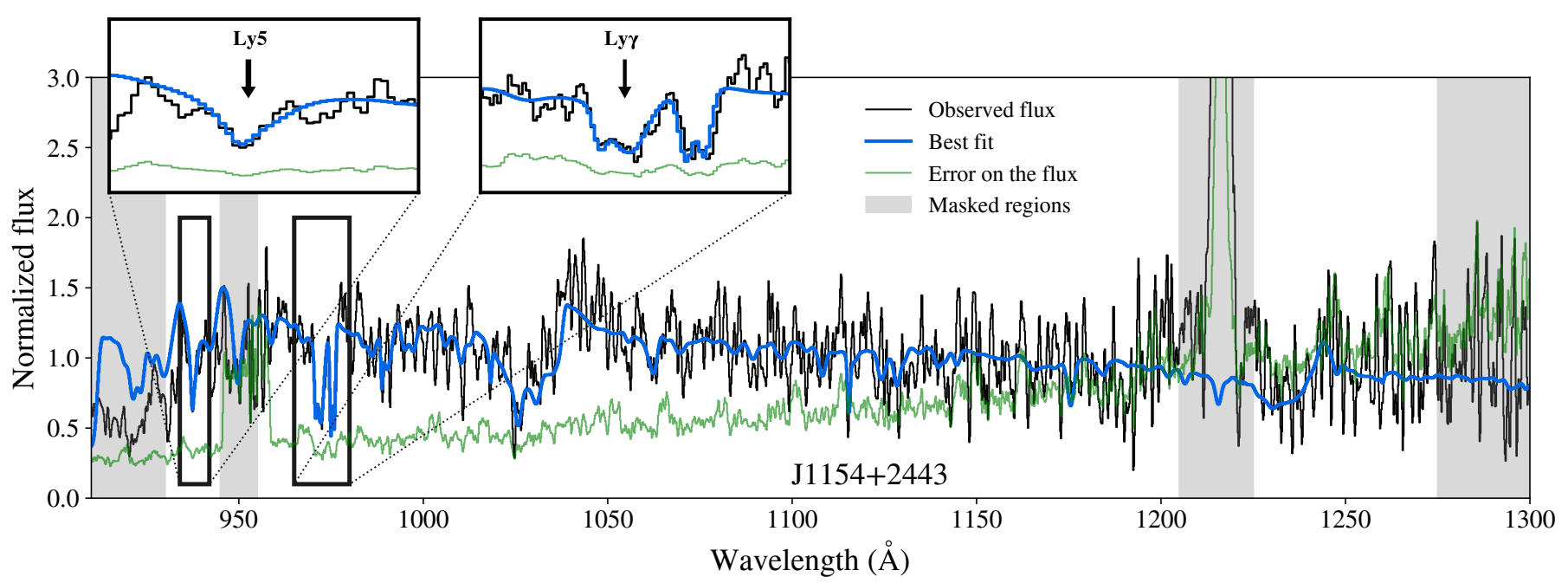

Fig. A.1. Fit (in blue solid line) of the COS G140L spectrum of the galaxy J1154+2443 (Izotov et al. 2018a). The black line is the observed flux included in the routine either to fit the stellar continuum or the ISM absorption lines. Gray regions are masked out during the fit. The flux error array appears in green. The top panels present a zoom on the fitted Lyman series lines.

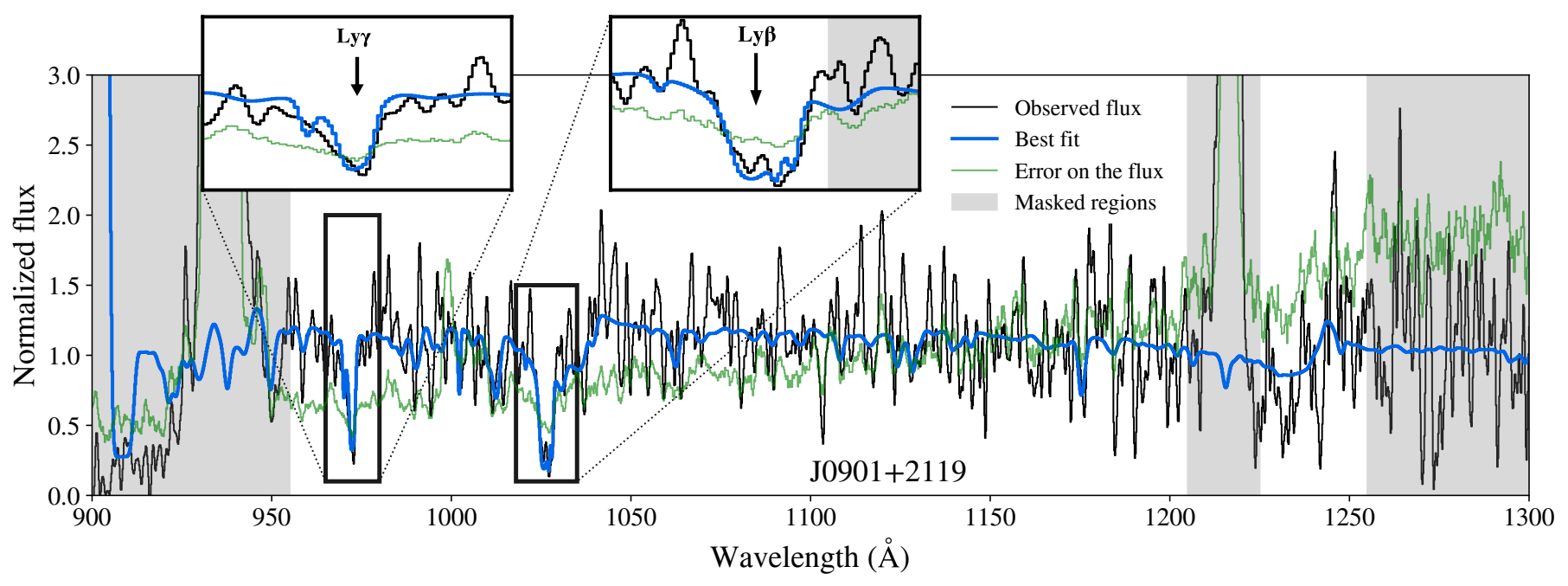

Fig. A.2. Same as Fig. A.1 but for the COS G140L spectrum of J0901+2119 (Izotov et al. 2018b).

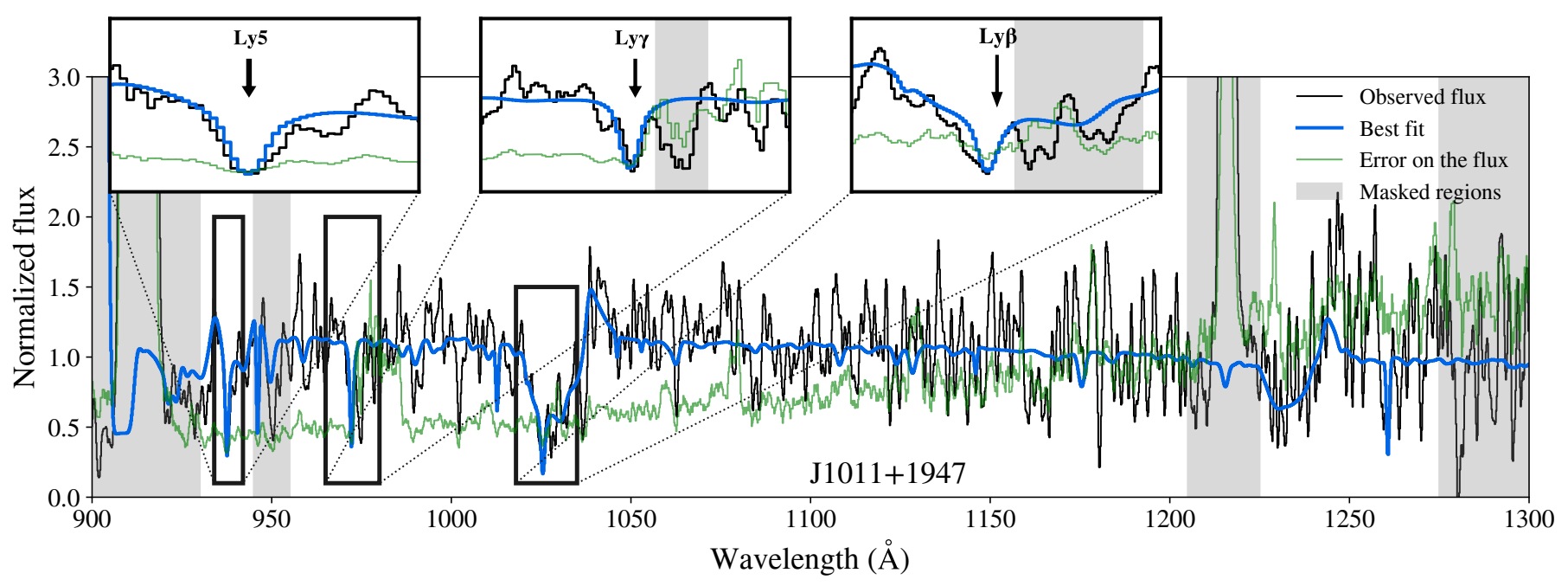

Fig. A.3. Same as Fig. A.1 but for the COS G140L spectrum of J1011+1947 (Izotov et al. 2018b). 


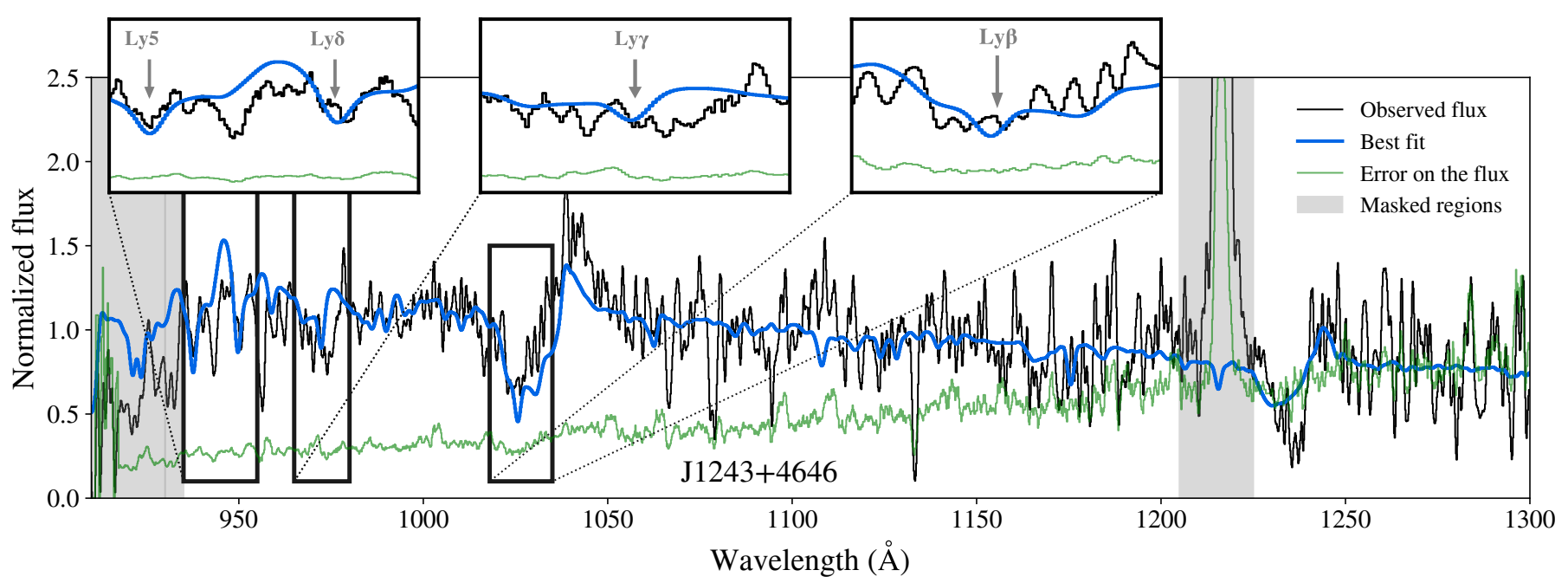

Fig. A.4. Same as Fig. A.1 but for the COS G140L spectrum of J1243+4646 (Izotov et al. 2018b). The best fit only includes the attenuated stellar continuum because the $\mathrm{H}_{\mathrm{I}}$ absorption lines were not found.

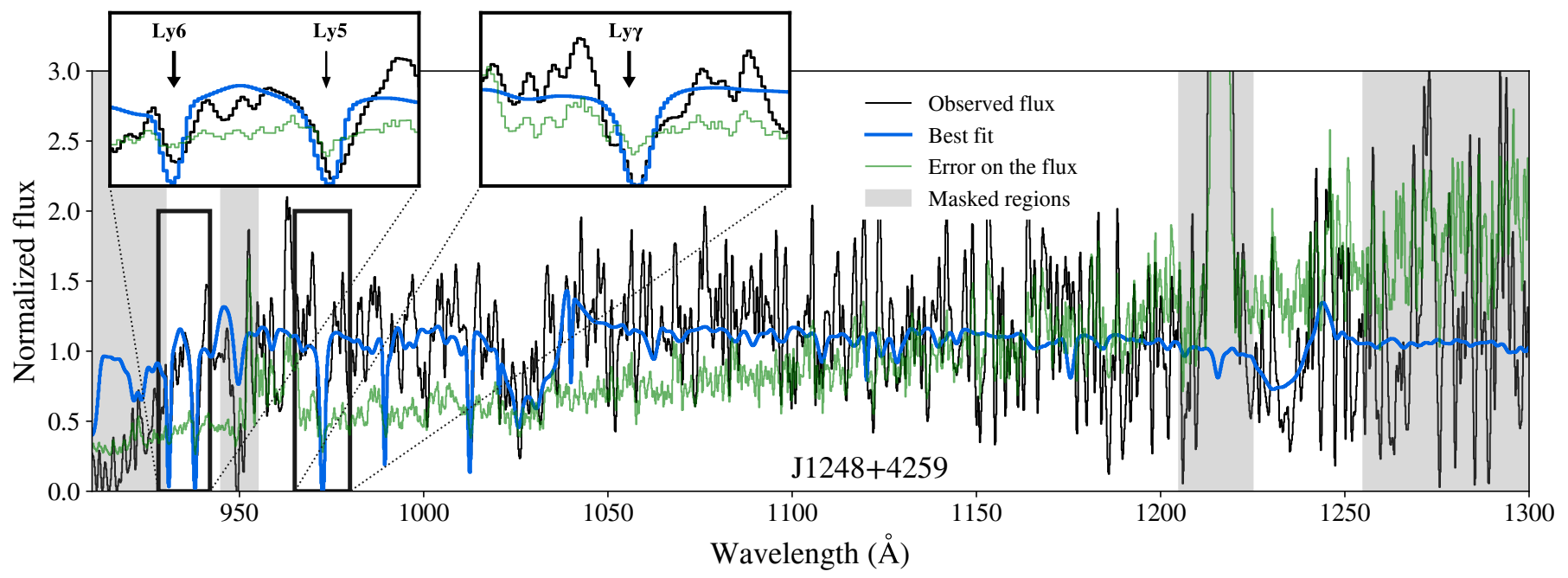

Fig. A.5. Same as Fig. A.1 but for the COS G140L spectrum of J1248+4259 (Izotov et al. 2018b).

\section{Appendix B: Tables}

\section{Appendix B.1: Lyman series covering fraction measurements}

Table B.1 lists the different measurements of the $\mathrm{H}_{\mathrm{I}}$ covering fractions, from the fits and from the residual flux (see details in Sect. 3.1.

\section{Appendix B.2: Lyman series velocity width measurements}

Table B.2 lists the measurements of the $\mathrm{H}_{\mathrm{I}}$ velocity width for the Lyman series in each galaxy (see details in Sect. 3.1).

\section{Appendix B.3: Significance levels of the trends reported in this work}

In this Section, we investigate the dependence of the significance levels of the trends reported in Sect. 4 on a few specific observations, or on different assumptions of the intrinsic Ly $\alpha \mathrm{H} \alpha$ flux ratio adopted to derive $f_{\text {esc }}(\operatorname{Ly} \alpha)$. Table $\mathrm{B} .3$ lists the $\sigma$ values quoted in the paper ( $\mathrm{Col}(2))$ and the new significance levels when one observation is excluded, quoted as a jackknife test interval (Col (3)), with the lowest significance and largest significance level obtained when one observation is removed from the sample. Col (3) shows that the lower bound is always larger than $2.5 \sigma$, except for three trends: $v_{\mathrm{Ly} \alpha}^{\text {red,rel }}-C_{f}(\mathrm{HI}), f_{\mathrm{esc}}^{\mathrm{obs}}(\mathrm{LyC})$ $-C_{f}(\mathrm{HI})$ and $\frac{\mathrm{F}_{\text {trough }}}{\mathrm{F}_{\text {cont }}}-C_{f}\left(\mathrm{HI}_{\mathrm{I}}\right)$. In the latter, the lowest $\sigma$ value is only moderately significant $(2 \sigma)$ and is obtained when the largest $f_{\text {esc }}(\mathrm{LyC})$ galaxy, $\mathbf{J} 1243+4646$, is excluded from the sample. We note that two of these trends suffer some scatter (illustrated in the Figs. 5 and 14 while the trend between $f_{\text {esc }}^{\text {obs }}(\mathrm{LyC})$ and $C_{f}\left(\mathrm{H}_{\mathrm{I}}\right)$ only has a limited number of irregularly-spaced points (Fig. 11). Future observations should confirm or rule out the existence of these trends.

In Col (4), we compute the new significance levels when $C_{f}(\mathrm{HI})$ is fixed to 0 for galaxies where we only measure an upper limit of the $C_{f}(\mathrm{HI})$. Overall, the significances vary by $\pm 0.5 \sigma$, except for the $\operatorname{EW}(\operatorname{Ly} \alpha)$ and $f_{\mathrm{esc}}(\operatorname{Ly} \alpha)$ correlations which reach 1.5 and $5.0 \sigma$, respectively. This is because the galaxies with an upper limit on $C_{f}(\mathrm{HI})$ ( 1 in our sample, 3 in the sample included in McKinney et al. 2019) have similar $f_{\text {esc }}(\operatorname{Ly} \alpha)$, but varying $\mathrm{EW}(\mathrm{Ly} \alpha)$. Hence, fixing their $C_{f}\left(\mathrm{H}_{\mathrm{I}}\right)$ to the same value strengthens the correlation with the $f_{\operatorname{esc}}(\operatorname{Ly} \alpha)$, but weakens the one with 
Table B.1. Measurement of $\mathrm{H}_{\mathrm{I}}$ covering fraction derived from the fits and from the residual flux of the individual Lyman series. See Sect. 3.1

\begin{tabular}{ccccc|cc||c}
\hline \hline $\begin{array}{c}\text { Galaxy name } \\
(1)\end{array}$ & $\begin{array}{c}\text { Ly } \beta \\
(2)\end{array}$ & $\begin{array}{c}\text { Ly } \gamma \\
(3)\end{array}$ & $\begin{array}{c}\text { Ly } \delta \\
(4)\end{array}$ & $\begin{array}{c}\text { Ly5 } \\
(5)\end{array}$ & $\begin{array}{c}\text { Depth } \\
(6)\end{array}$ & $\begin{array}{c}\text { Fits } \\
(7)\end{array}$ & $\begin{array}{c}\text { Final } \\
(8)\end{array}$ \\
\hline $\mathrm{J} 1243+4646$ & - & $0.200 \pm 0.121$ & $0.020 \pm 0.120$ & $0.140 \pm 0.120$ & $0.120 \pm 0.069$ & - & $<0.189$ \\
$\mathrm{~J} 1154+2443$ & - & $0.471 \pm 0.128$ & - & $0.474 \pm 0.184$ & $0.472 \pm 0.105$ & $0.404 \pm 0.152$ & $0.450 \pm 0.086$ \\
$\mathrm{~J} 1256+4509$ & $0.369 \pm 0.290$ & - & $0.530 \pm 0.165$ & $0.329 \pm 0.143$ & $0.410 \pm 0.101$ & $0.407 \pm 0.125$ & $0.409 \pm 0.079$ \\
$\mathrm{~J} 1152+3400$ & $0.619 \pm 0.088$ & - & - & - & $0.619 \pm 0.088$ & $0.629 \pm 0.069$ & $0.625 \pm 0.054$ \\
$\mathrm{~J} 1442-0209$ & $0.589 \pm 0.049$ & $0.471 \pm 0.068$ & - & - & $0.549 \pm 0.040$ & $0.621 \pm 0.120$ & $0.556 \pm 0.038$ \\
$\mathrm{~J} 0925+1409$ & $0.635 \pm 0.094$ & - & - & - & $0.635 \pm 0.094$ & $0.652 \pm 0.218$ & $0.638 \pm 0.086$ \\
$\mathrm{~J} 1011+1947$ & $0.693 \pm 0.376$ & $0.616 \pm 0.244$ & - & $0.820 \pm 0.210$ & $0.727 \pm 0.147$ & $0.644 \pm 0.265$ & $0.708 \pm 0.113$ \\
$\mathrm{~J} 1503+3644$ & $0.847 \pm 0.157$ & $0.723 \pm 0.129$ & $0.741 \pm 0.112$ & $0.744 \pm 0.113$ & $0.754 \pm 0.062$ & $0.611 \pm 0.110$ & $0.721 \pm 0.055$ \\
$\mathrm{~J} 1333+6246$ & $0.773 \pm 0.152$ & $0.870 \pm 0.103$ & $0.804 \pm 0.106$ & - & $0.826 \pm 0.066$ & $0.731 \pm 0.122$ & $0.804 \pm 0.058$ \\
J0901+2119 & $0.590 \pm 0.461$ & $0.742 \pm 0.247$ & - & - & $0.708 \pm 0.218$ & $0.539 \pm 0.257$ & $0.637 \pm 0.166$ \\
$\mathrm{~J} 1248+4259$ & - & $1.000 \pm 0.170$ & - & $0.903 \pm 0.157$ & $0.948 \pm 0.115$ & $0.980 \pm 0.241$ & $0.954 \pm 0.104$ \\
J0921+4509 & $0.769 \pm 0.116$ & - & - & - & $0.769 \pm 0.116$ & $0.754 \pm 0.111$ & $0.761 \pm 0.080$ \\
To11247-232 & $0.543 \pm 0.135$ & - & $0.644 \pm 0.115$ & $0.414 \pm 0.175$ & $0.564 \pm 0.078$ & $0.486 \pm 0.061$ & $0.518 \pm 0.046$ \\
J0926+4427 & $0.817 \pm 0.057$ & $0.807 \pm 0.087$ & - & - & $0.814 \pm 0.048$ & $0.673 \pm 0.040$ & $0.731 \pm 0.031$ \\
J1429+0643 & $0.955 \pm 0.061$ & - & - & - & $0.955 \pm 0.061$ & $0.898 \pm 0.071$ & $0.931 \pm 0.046$ \\
GP0303-0759 & - & - & - & - & - & $0.908 \pm 0.207$ & $0.908 \pm 0.207$ \\
GP1244+0216 & $0.985 \pm 0.211$ & $0.894 \pm 0.204$ & $1.000 \pm 0.292$ & - & $0.950 \pm 0.131$ & $0.909 \pm 0.357$ & $0.946 \pm 0.123$ \\
GP1054+5238 & $0.936 \pm 0.318$ & - & $0.891 \pm 0.203$ & $0.798 \pm 0.420$ & $0.889 \pm 0.158$ & $0.778 \pm 0.131$ & $0.823 \pm 0.101$ \\
GP0911+1831 & $0.718 \pm 0.361$ & $0.781 \pm 0.189$ & $0.825 \pm 0.198$ & $0.635 \pm 0.282$ & $0.765 \pm 0.116$ & $0.731 \pm 0.150$ & $0.752 \pm 0.092$ \\
SGAS J1226 & $1.000 \pm 0.010$ & $1.000 \pm 0.030$ & $0.981 \pm 0.057$ & $0.940 \pm 0.060$ & $0.998 \pm 0.009$ & $0.932 \pm 0.038$ & $0.994 \pm 0.009$ \\
SGAS J1527 & $0.858 \pm 0.141$ & $1.000 \pm 0.045$ & $1.000 \pm 0.087$ & $1.000 \pm 0.201$ & $0.990 \pm 0.038$ & $0.993 \pm 0.078$ & $0.990 \pm 0.034$ \\
Cosmic Eye & $1.000 \pm 0.043$ & $1.000 \pm 0.063$ & $1.000 \pm 0.033$ & $0.899 \pm 0.166$ & $0.998 \pm 0.024$ & $0.920 \pm 0.070$ & $0.990 \pm 0.023$ \\
\hline
\end{tabular}

Notes. (1) Galaxy name; (2)-(5) Residual flux of the individual Lyman series (from Ly $\beta$ to Ly5); (6) H I covering fraction from the residual flux of the individual Lyman series derived as the weighted average of the columns (2)-(5). Dashes indicate that these transitions were not observed/included owing to Milky Way absorption, geocoronal emission, or low S/N. (7) H I covering fraction obtained from the fitting method. (8) H I covering fraction of the galaxy, derived as the weighted average of the columns (6) and (7).

EW(Ly $\alpha)$. In Col (5), we excluded the three z 3 MegaSaura galaxies to investigate the impact they have on the significance of the trends. All the trends, except for $\operatorname{EW}(\mathrm{Ly} \alpha)-C_{f}(\mathrm{HI})$, remain significant at least at the $2.5 \sigma$ significance level.

Finally, we tested the impact of the Case-A/B assumptions on the trends that involve $f_{\mathrm{esc}}(\mathrm{Ly} \alpha)$ in Cols (6), (7) and (8). In the Case A recombination case (optically thin ISM), the intrinsic ratio of the Ly $\alpha$ and $\mathrm{H} \alpha$ flux can be 1.5 times higher (Osterbrock 1989 , the effective recombination coefficient of the Balmer emission lines is $\approx 1.5$ times higher than in the Case B assumption,) than in Case-B recombination (optically thick ISM, low-density case). For galaxies emitting ionizing photons, Case-B assumption (adopted in this work) is not likely valid, hence, an optimal approach would consist of scaling down all $f_{\text {esc }}(\operatorname{Ly} \alpha)$ values from LCEs by 1.5. Nevertheless, this is not trivial because some galaxies in our sample, or in the sample from McKinney et al. (2019) and Jaskot et al. (2019), do not have observations at $\lambda<912 \AA$ (unknown LyC leakage). Consequently, we choose to use a cut in the peak separation velocity of the Ly $\alpha$ profile to select galaxies with a Case-A ISM. The choice of $v_{\mathrm{Ly} \alpha}^{\text {sep }}$ is motivated by the strong observational correlation found between $f_{\mathrm{esc}}^{\mathrm{obs}}(\mathrm{LyC})$ and $v_{\mathrm{Ly} \alpha}^{\text {sep }}$ (Verhamme et al. 2017; Izotov et al. 2018b). Because low $f_{\text {esc }}(\mathrm{LyC})$ leakers might still be consistent with a Case-B recombination assumption, we select three cuts in $v_{\mathrm{Ly} \alpha}^{\text {sep }}$ of 400 , 300 and $200 \mathrm{~km} \mathrm{~s}^{-1}$, to successively select different sub-samples with larger $f_{\text {esc }}(\mathrm{LyC})$, and scaled-down their $f_{\mathrm{esc}}(\mathrm{Ly} \alpha)$ by a fac- tor 1.5. We found that the $f_{\text {esc }}(\operatorname{Ly} \alpha)$ trends are always significant at $\geq 2.5 \sigma$. Therefore, we conclude that variations in the intrinsic Ly $\alpha$-H $\alpha$ flux ratio should not affect the conclusions drawn in this work.

\section{Appendix C: Plots of $\mathrm{Ly} \alpha$ and $\mathrm{H}$ I absorption line profiles.}

Figures C.1 and C.2 show the $\operatorname{Ly} \alpha$ emission and $\operatorname{Ly} \beta$ (or $\operatorname{Ly} \gamma$ when $\operatorname{Ly} \beta$ is not observed) in velocity space for the 22 galaxies in our sample. 
Table B.2. Measurement of H I velocity width of the individual Lyman series. See Sect. 3.1

\begin{tabular}{|c|c|c|c|c|c|}
\hline Galaxy name & $\begin{array}{c}v_{\mathrm{Ly} \beta}^{\text {width }} \\
{\left[\mathrm{km} \mathrm{s}^{-1}\right]} \\
(2)\end{array}$ & $\begin{array}{c}v_{\mathrm{Ly} \gamma}^{\text {width }} \\
{\left[\mathrm{km} \mathrm{s}^{-1}\right]} \\
\text { (3) }\end{array}$ & $\begin{array}{c}v_{\mathrm{Ly} \delta}^{\text {width }} \\
{\left[\mathrm{km} \mathrm{s}^{-1}\right]} \\
(4)\end{array}$ & $\begin{array}{c}v_{\mathrm{Ly} 5}^{\text {width }} \\
{\left[\mathrm{km} \mathrm{s}^{-1}\right]} \\
(5)\end{array}$ & $\begin{array}{c}v_{\mathrm{H}_{\mathrm{I}}}^{\text {width }} \\
{\left[\mathrm{km} \mathrm{s}^{-1}\right]} \\
(6)\end{array}$ \\
\hline $\mathrm{J} 1243+4646$ & - & - & - & - & - \\
\hline $\mathrm{J} 1154+2443$ & - & $170 \pm 100$ & - & $170 \pm 170$ & $170 \pm 86$ \\
\hline $\mathrm{J} 1256+4509$ & $270 \pm 80$ & - & $220 \pm 90$ & $280 \pm 90$ & $250 \pm 50$ \\
\hline $\mathrm{J} 1152+3400$ & $420 \pm 60$ & - & - & - & $420 \pm 60$ \\
\hline J1442-0209 & $490 \pm 80$ & $280 \pm 70$ & - & - & $371 \pm 53$ \\
\hline J0925+1409 & $320 \pm 60$ & - & - & - & $320 \pm 60$ \\
\hline $\mathrm{J} 1011+1947$ & $420 \pm 80$ & $170 \pm 90$ & - & $170 \pm 130$ & $285 \pm 54$ \\
\hline $\mathrm{J} 1503+3644$ & $410 \pm 90$ & $380 \pm 100$ & $280 \pm 100$ & $340 \pm 100$ & $356 \pm 49$ \\
\hline $\mathrm{J} 1333+6246$ & $320 \pm 80$ & $280 \pm 100$ & $230 \pm 90$ & - & $280 \pm 51$ \\
\hline J0901+2119 & $320 \pm 110$ & $280 \pm 110$ & - & - & $300 \pm 78$ \\
\hline $\mathrm{J} 1248+4259$ & - & $260 \pm 90$ & - & $220 \pm 60$ & $232 \pm 50$ \\
\hline J0921+4509 & $440 \pm 20$ & - & - & - & $440 \pm 20$ \\
\hline Tol1247-232 & $530 \pm 140$ & - & $360 \pm 180$ & $430 \pm 150$ & $453 \pm 89$ \\
\hline J0926+4427 & $400 \pm 60$ & $360 \pm 70$ & - & - & $383 \pm 46$ \\
\hline $\mathrm{J} 1429+0643$ & $420 \pm 50$ & - & - & - & $420 \pm 50$ \\
\hline GP0303-0759 & $380 \pm 50$ & - & - & - & $380 \pm 50$ \\
\hline GP1244+0216 & $380 \pm 50$ & $370 \pm 205$ & - & - & $379 \pm 49$ \\
\hline GP1054+5238 & $580 \pm 50$ & - & $400 \pm 50$ & $460 \pm 50$ & $480 \pm 29$ \\
\hline GP0911+1831 & $410 \pm 60$ & $410 \pm 50$ & $370 \pm 20$ & $350 \pm 40$ & $374 \pm 16$ \\
\hline SGAS J1226 & $560 \pm 40$ & $600 \pm 80$ & - & $510 \pm 50$ & $548 \pm 29$ \\
\hline SGAS J1527 & $610 \pm 60$ & $520 \pm 70$ & - & $410 \pm 40$ & $480 \pm 30$ \\
\hline Cosmic Eye & $700 \pm 380$ & $740 \pm 210$ & $320 \pm 140$ & $450 \pm 220$ & $467 \pm 99$ \\
\hline
\end{tabular}

Notes. (1) Galaxy name; (2)-(5) Velocity width of the individual Lyman series (from Ly $\beta$ to Ly5); (6) H I velocity width from the residual flux of the individual Lyman series derived as the weighted average of the columns (2)-(5). Dashes indicate that these transitions were not observed/included owing to Milky Way absorption, geocoronal emission, low $\mathrm{S} / \mathrm{N}$, or irregular line shape. 
Table B.3. The impact of different assumptions on the reported significance level of observed trends.

\begin{tabular}{|c|c|c|c|c|c|c|c|}
\hline \multirow{2}{*}{$\begin{array}{c}\text { Trend } \\
\text { (1) }\end{array}$} & \multicolumn{7}{|c|}{ Significance level $(\sigma)$} \\
\hline & $\begin{array}{l}\text { All } \\
(2)\end{array}$ & $\begin{array}{c}\text { Jackknife interval } \\
\text { (3) }\end{array}$ & $\begin{array}{c}C_{f}^{\text {limit }}\left(\mathrm{H}_{\mathrm{I}}\right) \text { to } 0 \\
(4)\end{array}$ & $\begin{array}{c}\text { Low z } \\
(5)\end{array}$ & $\begin{array}{c}v_{\mathrm{Ly} \alpha}^{\mathrm{sep}}<400 \\
(6)\end{array}$ & $\begin{array}{c}v_{\mathrm{Ly} \alpha}^{\mathrm{sep}}<300 \\
(7)\end{array}$ & $\begin{array}{c}v_{\mathrm{Ly} \alpha}^{\mathrm{sep}}<200 \\
(8)\end{array}$ \\
\hline$v_{\mathrm{Ly} \alpha}^{\text {blue,rel }}-C_{f}(\mathrm{HI})$ & 3.0 & {$[2.5,3.5]$} & 2.5 & - & - & - & - \\
\hline$v_{\mathrm{Ly} \alpha}^{\text {red,rel }}-C_{f}(\mathrm{HI})$ & 3.0 & {$[2.0,3.5]$} & 3.0 & 2.5 & - & - & - \\
\hline$v_{\mathrm{Ly} \alpha}^{\text {sep }}-C_{f}(\mathrm{HI})$ & 3.0 & {$[2.5,3.5]$} & 3.5 & - & - & - & - \\
\hline $\mathrm{EW}(\mathrm{Ly} \alpha)-C_{f}(\mathrm{HI})$ & 2.5 & {$[2.5,4.0]$} & 1.5 & 2.0 & - & - & - \\
\hline$f_{\mathrm{esc}}(\mathrm{Ly} \alpha)-C_{f}(\mathrm{H} \mathrm{I})$ & 4.0 & {$[3.5,5.5]$} & 5.0 & 3.5 & 4.0 & 3.5 & 3.5 \\
\hline$f_{\mathrm{esc}}^{\mathrm{obs}}(\mathrm{LyC})-C_{f}(\mathrm{HI})$ & 3.0 & {$[2.0,3.0]$} & 3.5 & - & - & - & - \\
\hline$\frac{\mathrm{F}_{\text {trough }}}{\mathrm{F}_{\text {cont }}}-C_{f}(\mathrm{H} \mathrm{I})$ & 3.0 & {$[2.0,4.5]$} & 2.5 & - & - & - & - \\
\hline $\mathrm{EW}(\operatorname{Ly} \alpha)-v_{\mathrm{H}_{\mathrm{I}}}^{\text {width }}$ & 4.0 & {$[3.5,4.0]$} & - & 3.0 & - & - & - \\
\hline$f_{\mathrm{esc}}(\operatorname{Ly} \alpha)-v_{\mathrm{HI}}^{\text {width }}$ & 3.0 & {$[2.5,3.5]$} & - & 2.5 & 2.5 & 3.0 & 2.5 \\
\hline$f_{\mathrm{esc}}(\mathrm{Ly} \alpha)-10^{-0.4 A(1216 \AA)}$ & 3.0 & {$[2.5,3.5]$} & - & 2.5 & 2.5 & 2.5 & 2.5 \\
\hline$f_{\mathrm{esc}}^{\mathrm{obs}}(\mathrm{LyC})-10^{-0.4 A(912 \AA)}$ & 3.0 & {$[2.5,3.0]$} & - & - & - & - & - \\
\hline$f_{\text {esc }}^{\text {obs }}($ LyC $)-\frac{\mathrm{F}_{\text {trough }}}{F_{\text {cont }}}$ & 3.5 & {$[2.5,4.5]$} & - & - & - & - & - \\
\hline
\end{tabular}

Notes. (1) Tested correlation between the two variables, reported in Sect. 4. (2) trend significance level when all values are included, and when $C_{f}\left(\mathrm{H}_{\mathrm{I}}\right)$ is fixed to the upper limit in galaxies with only an upper bound. These values correspond to the significance levels quoted in the paper. (3) The interval of $\sigma$ derived by performing a jackknife test; (4) same as (2) but fixing $C_{f}\left(\mathrm{H}_{\mathrm{I}}\right)$ to 0 in galaxies with only a upper bound to the H I covering fraction; (5) same as (2), but only including galaxies with $\mathrm{z}<0.5$; (6)-(8) significance level of the $f_{\text {esc }}(\operatorname{Ly} \alpha)$ trend when $f_{\text {esc }}(\operatorname{Ly} \alpha)$ is scaled-down by a factor of 1.5 for galaxies with (6) $v_{\mathrm{Ly} \alpha}^{\text {sep }}<400 \mathrm{~km} \mathrm{~s}^{-1}$; (7) $v_{\mathrm{Ly} \alpha}^{\text {sep }}<300 \mathrm{~km} \mathrm{~s}^{-1}$ and (8) $v_{\mathrm{Ly} \alpha}^{\text {sep }}<200 \mathrm{~km} \mathrm{~s}^{-1}$. All significance level tests include the sample from McKinney et al. (2019) and Jaskot et al. (2019) when possible. Dashes indicate that the significance level remained unchanged with respect to (2) because the conditions applied did not affect the sample included. 

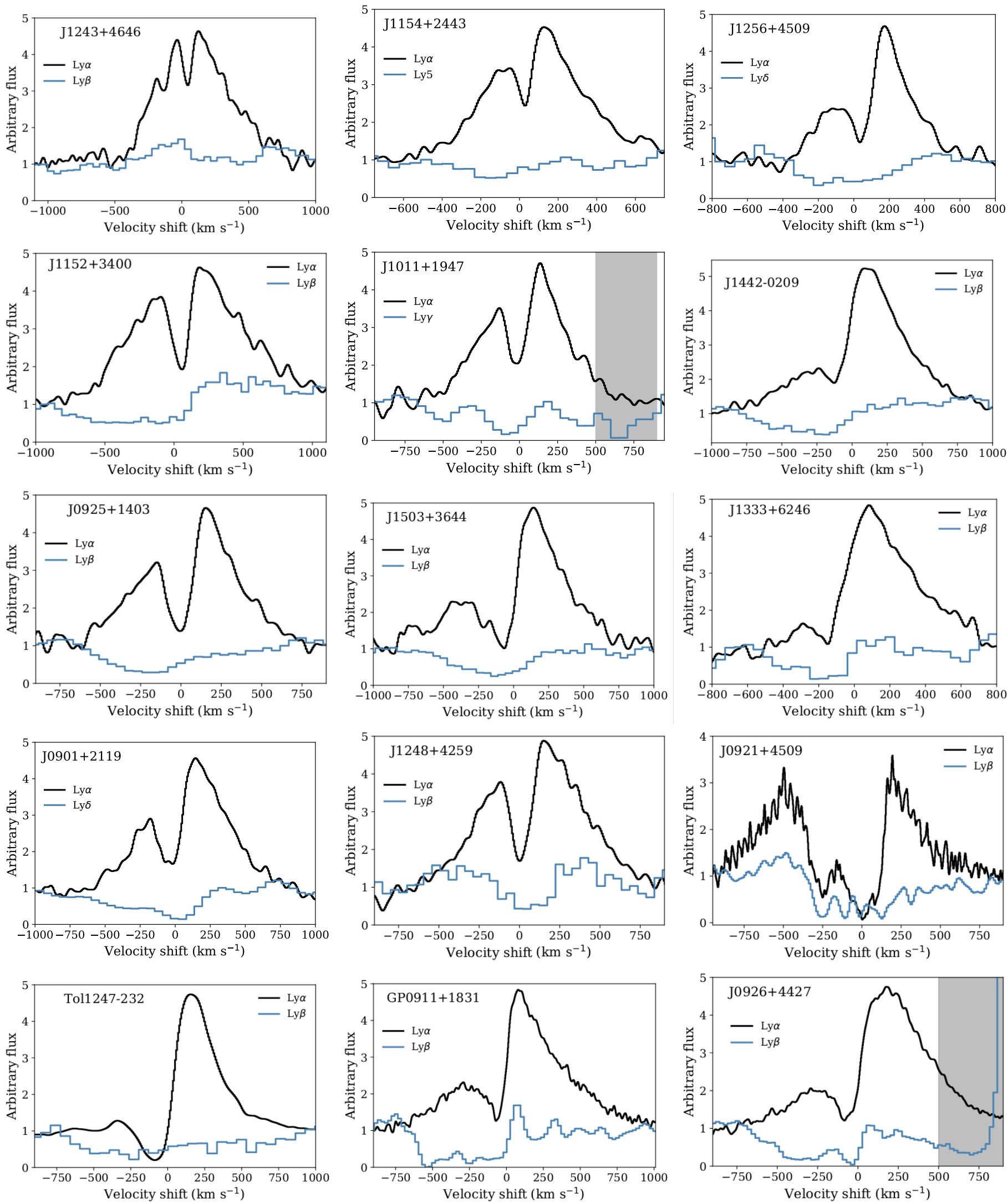

Fig. C.1. Plot of the Ly $\alpha$ emission and the observed H I Lyman series line with the larger $\mathrm{S} / \mathrm{N}$ in velocity space for 15 of the 22 galaxies in our sample. The Ly $\alpha$ flux has been smoothed to the resolution of the Lyman series, and scaled down by an arbitrary power law for display purpose. Gray shaded regions show contamination from geocoronal emission or Milky Way absorption lines next to the $\mathrm{H}_{\mathrm{I}}$ absorption line. 

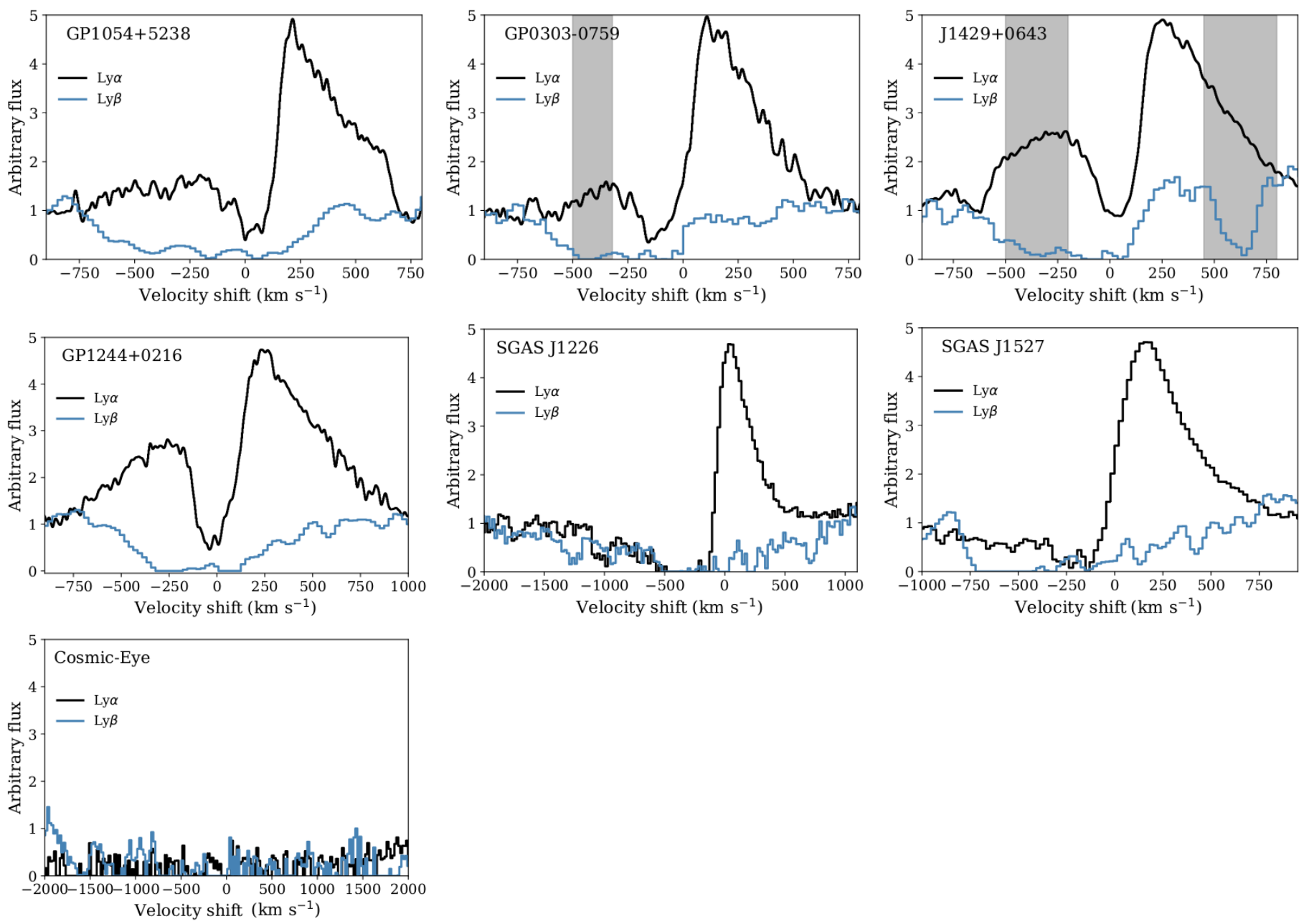

Fig. C.2. Same as Fig. C.1 for the 7 remaining galaxies. 